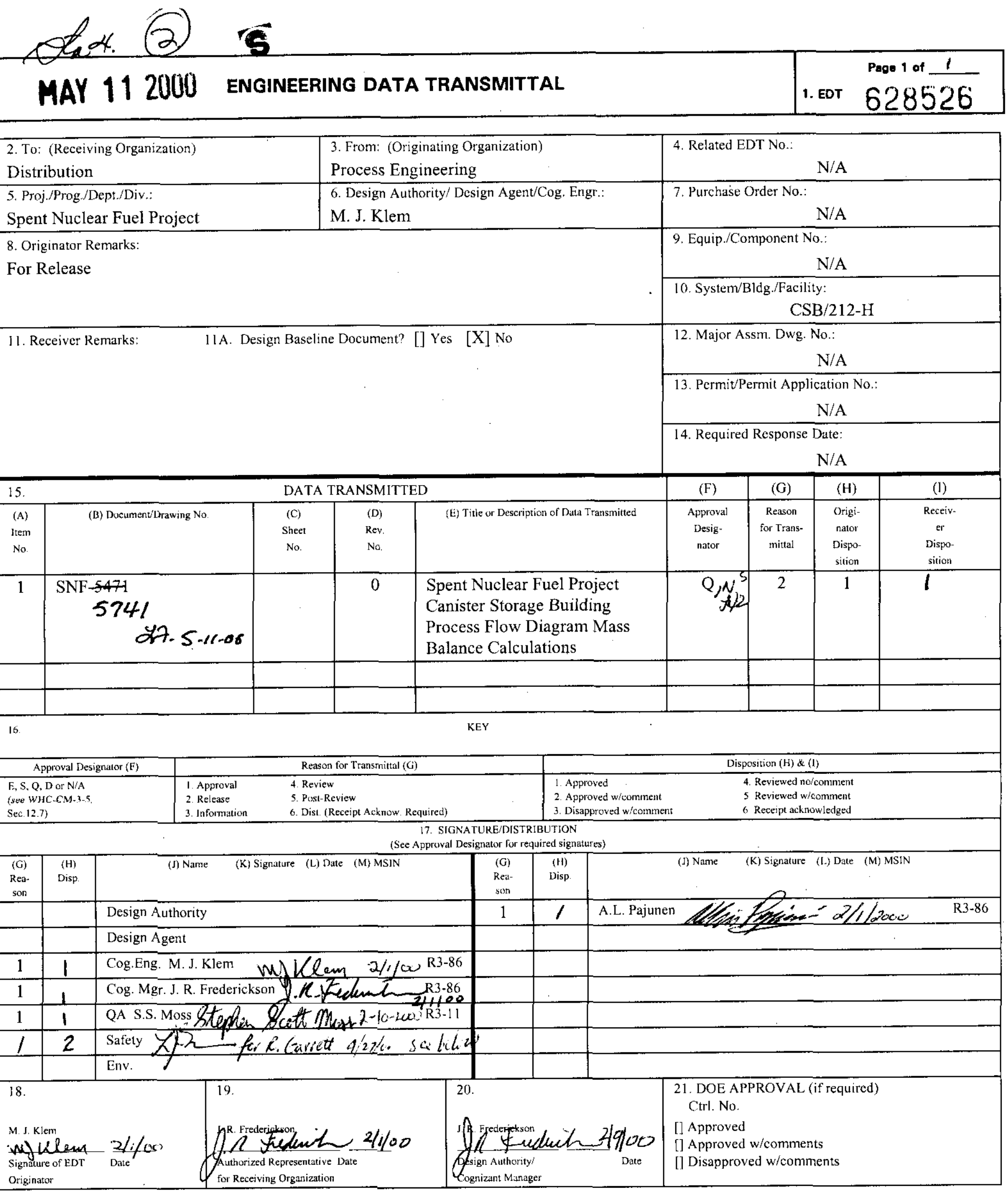

BD-7400-172-2 (05/96) GEF097

NS - This change cion thot the

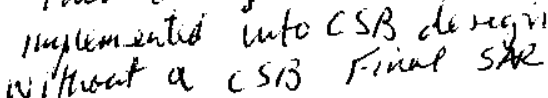

revisia 105 


\section{Spent Nuclear Fuel Project Canister Storage Building Process Flow Diagram Mass Balance Calculations}

Prepared for the U.S. Department of Energy

Assistant Secretary for Environmental Management

Project Hanford Management Contractor for the

U.S. Department of Energy under Contract DE-AC06-96RL13200

Fluor Hanford

P.O. Box 1000

Richland, Washington 


\section{Spent Nuclear Fuel Project Canister Storage Building Process Flow Diagram Mass Balance Calculations}

M. J. Klem

Cogema Engineering Corporation

A. L. Pajunen

Fluor Hanford

Date Published

May 2000

Prepared for the U.S. Department of Energy

Assistant Secretary for Environmental Management

Project Hanford Management Contractor for the

U.S. Department of Energy under Contract DE-AC06-96RL13200

Fluor Hanford

P.O. Box 1000

Richland, Washington
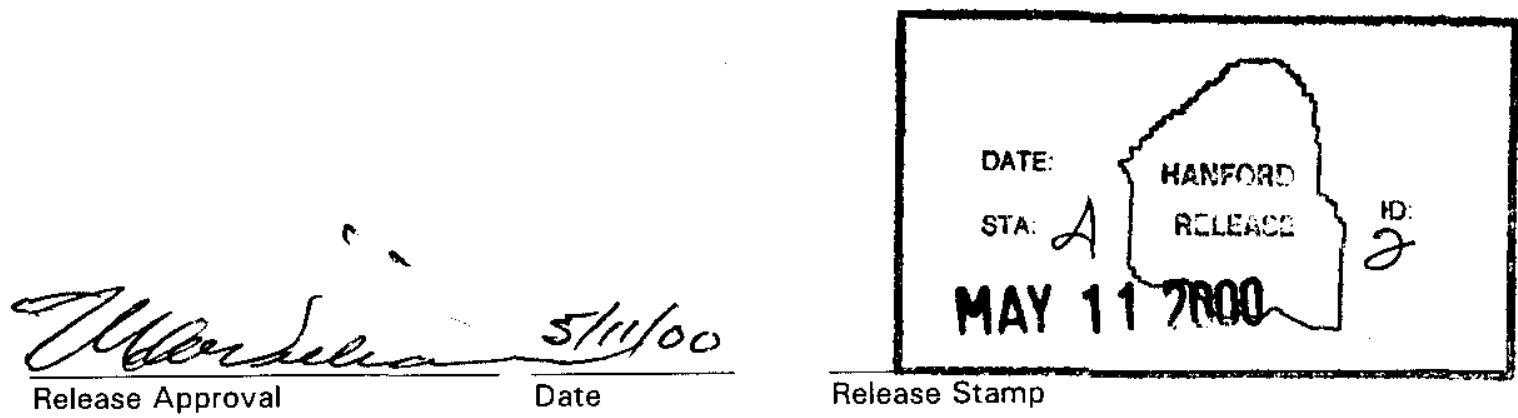

Release Stamp 
TRADEMARK DISCLAIMER

Reference herein to any specific commercial product, process, or service by trade name, trademark, manufacturer, or otherwise, does not necessarily constitute or imply its endorsement, recommendation, or favoring by the United States Government or any agency thereof or its contractors or subcontractors.

This report has been reproduced from the best available copy. Available in paper copy and microfiche.

Available electronically at http://www.doe.gov/bridge. Available for a processing fee to the U.S. Department of Energy and its contractors, in paper, from:

U.S. Department of Energy

Office of Scientific and Technical Information P.O. Box 62

Oak Ridge, TN 37831-0062

phone: $865-576-8401$

fax: 865-576-5728

email: reports@adonis.osti.gov(423) 576-8401

Total Pages: $\quad 53$

SNF- 5741 , nenO 


\section{TABLE OF CONTENTS}

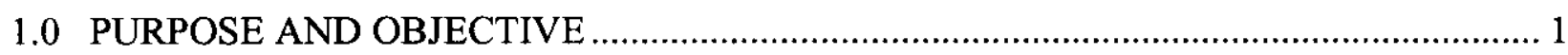

2.0 SUMMARY OF FINAL RESULTS AND CONCLUSIONS ........................................... 1

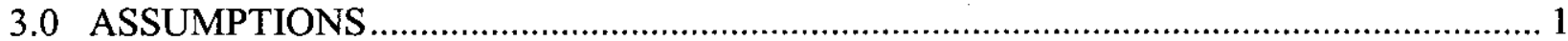

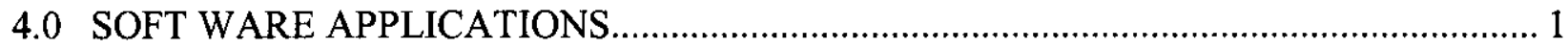

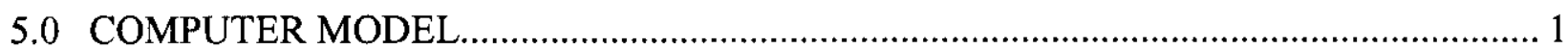

6.0 INPUT DATA

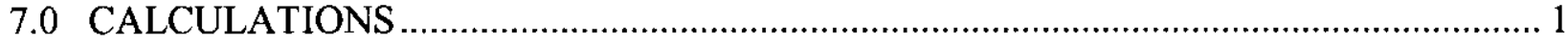

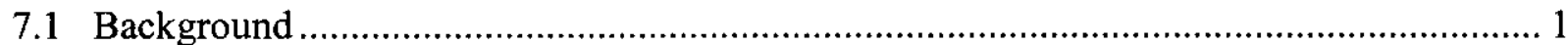

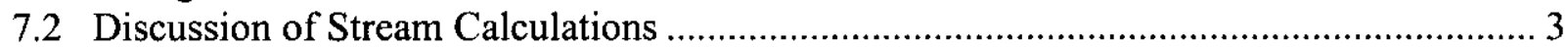

7.2.1 IC-003 Vacuum Dried Fuel In MCO/Cask Delivered to CSB ................................... 3

7.2.2 IC-006 Empty MCO Loaded in Cask For Transport to K Basins............................... 5

7.2.3 C-3 MCO/Cask in Cask Receiving Pit.................................................................... 5

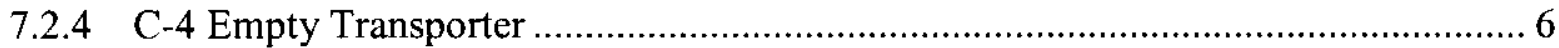

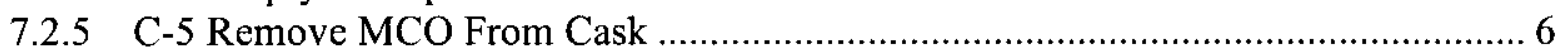

7.2.6 C-6 Move MCO From Transport Cask to Weld Station in MHM.................................. 6

7.2.7 C-7 Move Short Term Monitored MCO to Vault in MHM..................................... 8

7.2.8 C-8 Short Term Monitored MCO in Storage Tube ................................................. 9

7.2.9 C-9 Cask Receiving Pit Containment Tent Offgas ................................................ 9

7.2.10 C-10 Cask Receiving Pit Containment Tent Replacement Filters.............................. 9

7.2.11 C-11 Spent Cask Receiving Pit HEPA Filters …………....................................... 9

7.2.12 C-12 Short Term Monitored MCOs From Sampling to Storage Tube ...................... 9

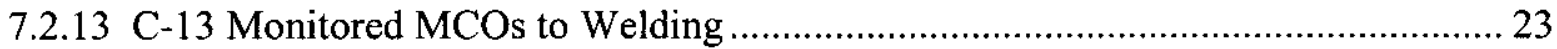

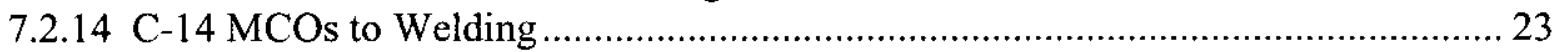

7.2.15 C-15 MCOs to Canister Cover Cap Welding ………............................................... 23

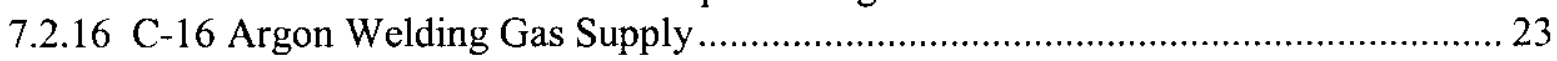

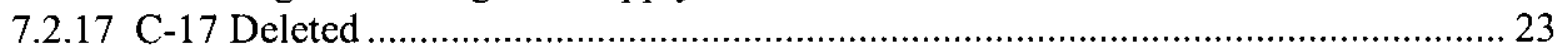

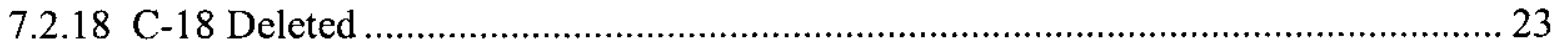

7.2.19 C-19 MCO Welding/Grinding Waste .................................................................. 24

7.2.20 C-20 MCO Weld Preparation and Examination Waste ............................................... 25

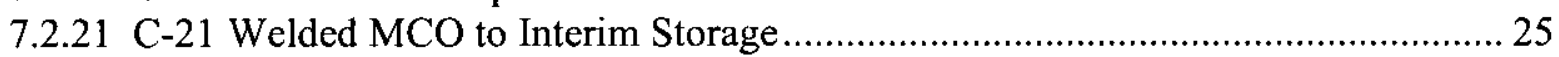

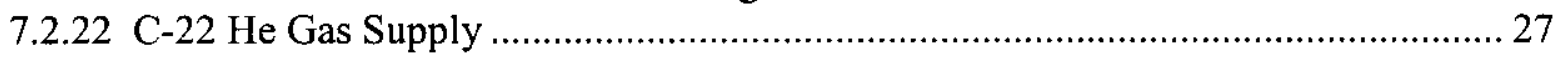

7.2.23 C-23 Monitored MCO Exhaust Gas …………............................................... 27

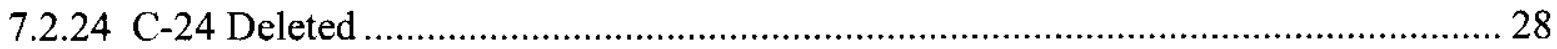

7.2.25 C-25 Annex Room Air to Weld Station Exhaust................................................... 28

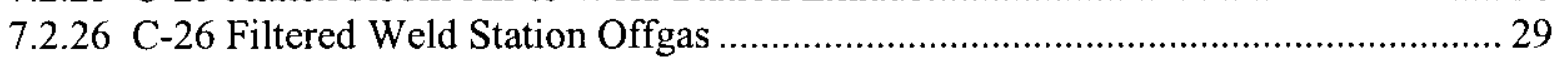

7.2.27 C-27 Weld Station Spent HEPA Filter Waste .......................................................... 29

7.2.28 C-28 Replacement Filters for Weld Station ......................................................... 29

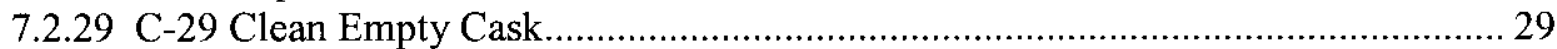

7.2.30 C-30 Cask Decontamination Material ................................................................... 29

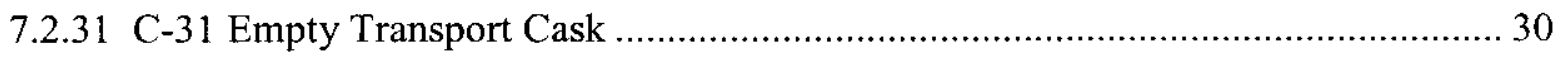

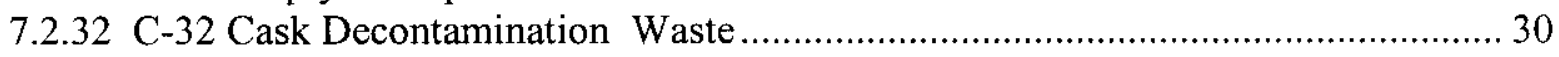

7.2.33 C-33 Miscellaneous Decontamination Material ..................................................... 30

7.2.34 C-34 Miscellaneous Decontamination Waste........................................................ 30

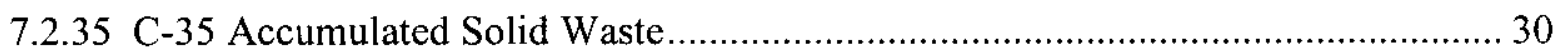




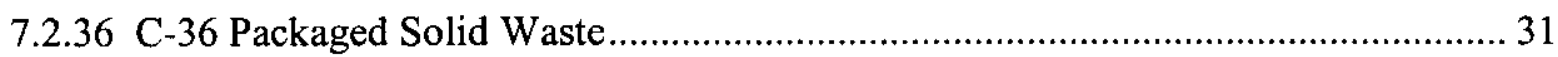

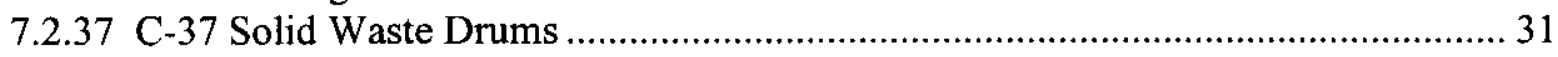

7.2.38 C-38 Cask Service System and Weld Station Exhaust ............................................ 31

7.2.39 C-39 MHM Ventilation Exhaust..................................................................... 31

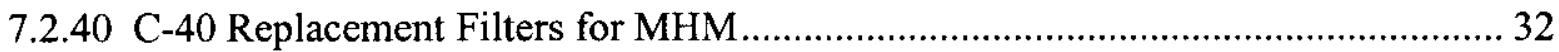

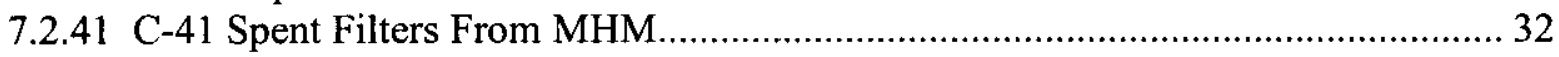

7.2.42 C-42 Operating Area Ventilation Exhaust .............................................................. 32

7.2.43 C-43 Spent HEPA Filter From Operating Area ......................................................... 33

7.2.44 C-44 Replacement HEPA Filter for Operating Area .............................................. 33

7.2.45 C-45 Spent HEPA Filters From Support Building ………................................. 33

7.2.46 C-46 Replacement HEPA Filters for Support Building ………................................. 33

7.2.47 C-47 Cask Service Exhaust System ......................................................................... 33

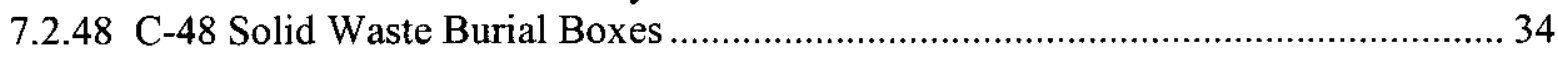

7.2.49 C-49 Accumulated Filter Waste........................................................................ 34

7.2.50 C-50 Replacement Sample HEPA Filter................................................................ 34

7.2.51 C-51 Spent HEPA Filter from Sample Hood............................................................ 34

7.2.52 C-52 Helium Refilled Monitored MCOs to Welding ............................................... 34

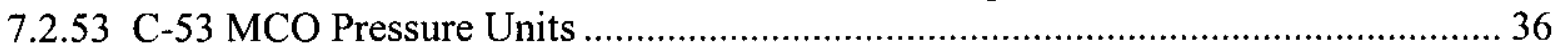

7.2.54 IC-083 Filtered and Monitored Exhaust From CSB ……………………............. 36

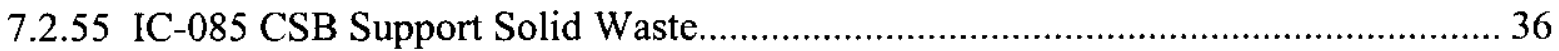

7.2.56 IC-087 New MCO Shell for Cask Loading ……................................................... 36

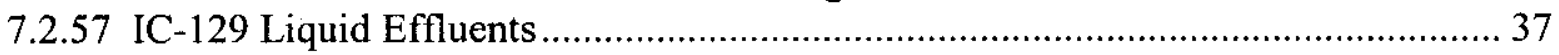

7.2.58 IC-233 MCO Cover Cap, Plug, and Plate ............................................................... 38

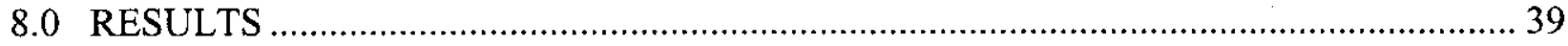

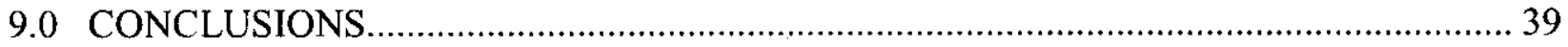

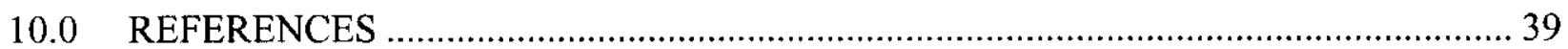

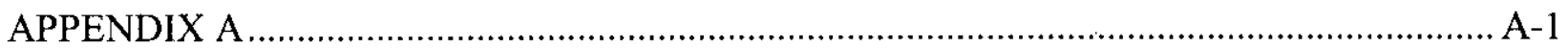




\section{LIST OF TABLES}

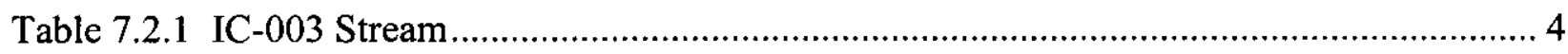

Table 7.2.2 Stream C-6

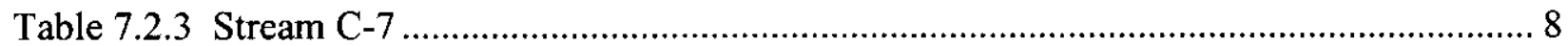

Table 7.2.4 Stream C-12 (Before 100 PSI Pressure Sending/Readout Unit Replacement)......... 10

Table 7.2.5 Summary of Monitored MCO Sampling ............................................................... 11

Table 7.2.6 Estimated Data from Best Estimate Single Scrap Basket Case ............................... 11

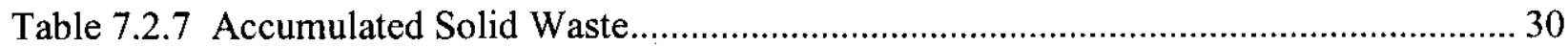

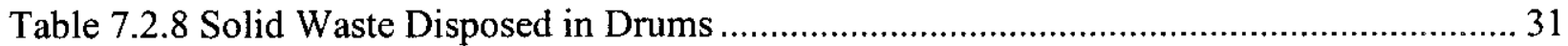

Table 7.2.9 Cask Service System and Weld Station Exhaust .................................................. 31

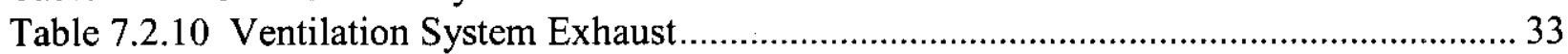

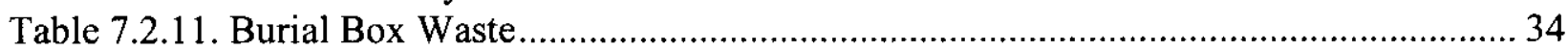

Table 7.2.12 Stream C-52 (After Pressure Sending/Readout Unit Replacement) ....................... 35

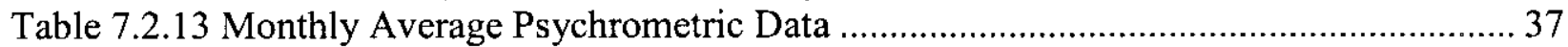

Table 7.2.14 Estimated Compressed Air Condensate.................................................................. 38

\section{LIST OF ACRONYMS}

$\begin{array}{ll}\text { CSB } & \text { Canister Storage Building } \\ \text { CVD } & \text { Cold Vacuum Drying } \\ \text { CVDF } & \text { Cold Vacuum Drying Facility } \\ \text { HVAC } & \text { Heating, Ventilation Air Conditioning } \\ \text { LERF } & \text { Liquid Effluent Retention Facility } \\ \text { MCO } & \text { Multi-Canister Overpack } \\ \text { MHM } & \text { MCO Handling Machine } \\ \text { MTU } & \text { Metric Tons Uranium } \\ \text { OBSFD } & \text { Operation Block Sequence Flow Diagram } \\ \text { PFD } & \text { Process Flow Diagram } \\ \text { SNF } & \text { Spent Nuclear Fuel }\end{array}$


SNF-5741, Rev. 0

\subsection{PURPOSE AND OBJECTIVE}

The purpose of these calculations is to develop the material balances for documentation of the Canister Storage Building (CSB) Process Flow Diagram (PFD) and future reference. The attached mass balances were prepared to support revision two of the PFD for the CSB. The calculations refer to diagram $\mathrm{H}-2-825869$.

\subsection{SUMMARY OF FINAL RESULTS AND CONCLUSIONS}

See Appendix A for final results.

\subsection{ASSUMPTIONS}

See assumptions page in Appendix A.

\subsection{SOFT WARE APPLICATIONS}

No software applications were used.

\subsection{COMPUTER MODEL}

A Microsoft Excel spreadsheet was used to display material balances and verify hand calculations.

\subsection{INPUT DATA}

See Section 7.2 and Appendix A.

\subsection{CALCULATIONS}

\subsection{Background}

During the K Basin fuel removal process, the combined K Basins inventory of SNF from the N Reactor, which totals 2, 103 MTU (metric tons uranium) per Reilly (1998) is cleaned, re-racked, and loaded into 398 (Goldmann 2000) mechanically sealed multi-canister overpacks (MCOs), and transferred to the cold vacuum drying (CVD) Facility within individual casks. The CVD Facility drains and dries the SNF in place within each Cask/MCO assembly for transport from the $100 \mathrm{~K}$ Area to the CSB, where the MCOs are removed from their Transport Casks, staged for installation of welded closure caps, welded, and interim stored for up to 40 years. Following removal of an MCO from its Transport Cask, the empty cask is fitted with a new, empty MCO and returned to the $\mathrm{K}$ Basins, completing one process cycle.

The fuel conditioning processes (cleaning/re-racking at the $\mathrm{K}$ Basins and drying at the CVD Facility) are designed to produce MCOs that contain no more than $200 \mathrm{~g}$ of free water (i.e., not chemically bound as water of hydration) and not more than $1056 \mathrm{~g}$ of chemically bound water (1256 g total design basis value). Upon completion of the drying process, the Cask/MCO 
assembly is cooled by circulating chilled, deionized water through the Cask/MCO annulus for five hours, after which the MCO is backfilled with helium to 11 psig (9.5 psig to 12.5 psig worst case actual pressure range, after correction for calibration errors, etc.). The CVD Facility equipment is disconnected from the MCO process ports, the cooling water is drained from the Cask/MCO annulus, the annulus is dried by an instrument air purge, the top of the MCO shield plug is wiped dry, and the cover plates are installed on each MCO port. The cover plate seals and the shield plug's main seal are then helium leak tested to ensure that their total combined leakage path does not exceed $1 \times 10^{-5} \mathrm{std} \mathrm{cm}^{3} / \mathrm{s}$, as defined by ANSI N14.5. Prior to installing the cask lid, the CVD Facility decontaminates and/or surveys all surfaces that will be exposed when the lid is removed at the CSB. Following installation of the cask lid, the CVD Facility decontaminates and (re)surveys all accessible surfaces of the cask and cask lid to comply with transport requirements and secures the cask for transport. The end product is a Cask/MCO assembly consisting of a thermally stable, helium inerted, mechanically sealed MCO (pressure relief is neither required nor provided) within a non-inerted, mechanically sealed Transport Cask.

The five hour pre-backfill cooling step and the 9.5 psig worst case minimum backfill pressure are designed to provide a minimum void space MCO (500L) with at least 31.0 gmol of helium. The minimum initial helium inventory is designed, in conjunction with the leak test acceptance criterion and associated helium loss calculations (Sherrell 1999), to support the oxygen gettering calculations of Duncan and Plys (1998), which assume that all MCOs will retain $\sim 30$ gmol of helium below their mechanically sealed shield plugs after 40 years of storage. The cool-down, backfill, and leak testing operations are more than adequate to ensure that all MCOs retain positive gage pressures (thus preventing ingress of air) at temperatures down to $-31^{\circ} \mathrm{C}$ (Sherrell 1999), and also ensure that hydrogen concentrations within standard, vented CSB storage tubes containing two mechanically sealed MCOs will never exceed $1 \mathrm{vol} \%$.

At the CSB, all 398 MCOs are welded, sealed closed, and interim stored until a decision on final disposition is reached and implemented. Argon is the cover gas and helium is the back gas during gas tungsten arc welding of the MCO cover cap assembly. Approximately six MCOs will undergo short term monitoring for a period of about two years before they are welded and sealed closed. Each monitored MCO is sampled a total of eight times during the two year operating period. After each sampling, the monitored MCO is reinerted with helium to replace the gas lost during sampling. The MCO sample cart is used to gas sample and reinert the monitored MCOs. The sample cart is purged with helium to remove air before MCO sampling and dilute the hydrogen after sampling for exhausting to the ventilation system.

Before welding of the monitored MCOs, the 100 psi pressure sending (located in the shield plug) is replaced with a 600 psi pressure sending unit. The monitored MCOs are vented through the internal filter and sample hood to atmospheric pressure, purged with helium during unit replacement, and then reinerted with helium to the same pressure. A 600 psi pressure readout unit is added to the cover cap of all 398 MCOs after welding. 
Condensate liquid waste from the compressed air system and the heating, ventilation air conditioning (HVAC) system is collected and loaded into 55-gallon drums for disposal at the Liquid Effluent Retention Facility (LERF) or to the ground, in accordance with SNF process standards. Solid waste is collected and loaded into 55-gallon drums or burial boxes for disposal as low level waste.

The following stream calculations are presented on a project total basis and based on the best estimate case for an MCO containing one scrap basket and four Mk IV fuel baskets.

\subsection{Discussion of Stream Calculations}

\subsubsection{IC-003 Vacuum Dried Fuel In MCO/Cask Delivered to CSB}

This is an input stream originating from H-1-81168, Rev. 3 cask transport PFDs. The stream mass is listed below: 
Table 7.2.1 IC-003 Stream

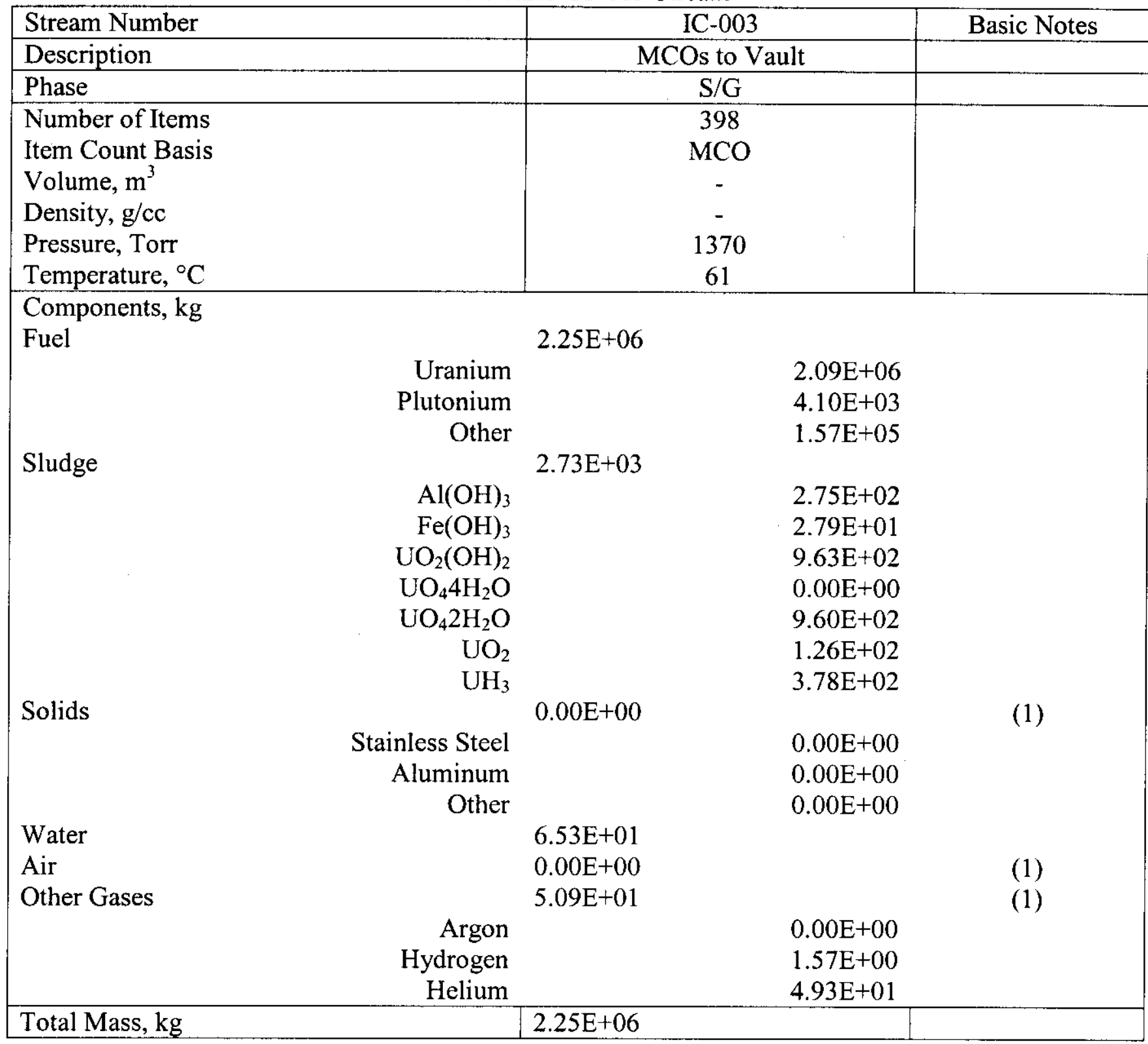

(1) Material balance neglects air within the cask void space as well as the material of construction of the cask and MCO. The container is not part of the mass balance. Page J-11 of Duncan and Plys (1998) and Table 2-1 of Pajunen (2000) are the source of information for temperature, pressure, and composition of the MCO internal gas.

The initial hydrogen concentration of $6.0 \mathrm{vol} \%$ at time zero is based on Duncan and Plys (1998) An initial fill of $31.0 \mathrm{gmol} \mathrm{He} / \mathrm{MCO}$ from Pajunen (2000) is used for this flowsheet (see Section 7.1). 
The following calculations are part of the cask/transport PFD from Klem and Pajunen (2000). The helium, hydrogen, and gas pressure calculations are repeated for background since they are used in subsequent calculations. The oxygen is negligible due to the gettering reaction with uranium metal based on Duncan and Plys (1998).

Calculate masses at time zero:

$0.06 \mathrm{H}_{2}=\mathrm{X}$ gmol H $\mathrm{H}_{2} /\left(\mathrm{X}\right.$ gmol $\mathrm{H}_{2}+31.0$ gmol He $)$ based on Duncan and Plys (1998) and Pajunen (2000)

Mass $\mathrm{H}_{2}(\mathrm{X})=0.06 \times 31.0 / 0.94=1.978 \mathrm{E}+00 \mathrm{gmol} \mathrm{H} / \mathrm{MCO}$

Total $\mathrm{H}_{2}$ mass at time zero $=1.978 \mathrm{E}+00 \mathrm{gmol} / \mathrm{MCO} \times 2 \mathrm{~g} \mathrm{H}_{2} / \mathrm{gmol} \times 398 \mathrm{MCOs} \times 0.001 \mathrm{~kg} / \mathrm{g}=$ $1.57 \mathrm{E}+00 \mathrm{~kg}$

Mass water/MCO at time zero $=<2.00 \mathrm{E}+02 \mathrm{~g} \mathrm{H}_{2} \mathrm{O}-1.98 \mathrm{E}+00 \mathrm{gmol} \mathrm{H}_{2} \times 1 \mathrm{gmol} \mathrm{H}_{2} \mathrm{O} / \mathrm{gmol} \mathrm{H}_{2} \mathrm{x}$ $18 \mathrm{~g} \mathrm{H}_{2} \mathrm{O} / \mathrm{gmol}=1.64 \mathrm{E}+02 \mathrm{~g} / \mathrm{MCO}$

Total water mass at time zero $=1.64 \mathrm{E}+02 \mathrm{~g} / \mathrm{MCO} \times 398 \mathrm{MCOs} \times 0.001 \mathrm{~kg} / \mathrm{g}=\mathbf{6 . 5 3 E}+\mathbf{0 1} \mathbf{~ k g}$

Mass helium (based on initial fill) $=31.0 \mathrm{gmol} / \mathrm{MCO} \times 4 \mathrm{~g} \mathrm{He} / \mathrm{gmol} \times 398 \mathrm{MCOs} \times 0.001 \mathrm{~kg} / \mathrm{g}=$ $4.93 \mathrm{E}+01 \mathrm{~kg}$

Time zero $\mathrm{MCO}$ gas pressure based on average temperature of fuel and $500 \mathrm{~L}$ void volume $=$ $\left(1.98 \mathrm{gmol} \mathrm{H} \mathrm{H}_{2}+31.0 \mathrm{gmol} \mathrm{He}\right) \times 0.0820 \mathrm{~L} \mathrm{~atm} / \mathrm{gmol} \mathrm{K} \times 334 \mathrm{~K} / 500 \mathrm{~L}=1.80 \mathrm{~atm}=26.5 \mathrm{psia}=$ 1370 torr

Private communication with M. G. Plys of Fauske and Associates reports that the average fuel temperature is more representative of the MCO gas temperature than the scrap temperature used in Duncan and Plys (1998). Therefore, the average fuel temperature is used as the MCO gas temperature. The 500L void volume is based on the one scrap basket and best estimate case MCO.

\subsubsection{IC-006 Empty MCO Loaded in Cask For Transport to K Basins}

A total of 398 empty MCOs are transported to the K Basins for loading with fuel and scrap. Air within the empty containers and the containers themselves are not included in the material balance.

\subsubsection{C-3 MCO/Cask in Cask Receiving Pit}

The $\mathrm{MCO}$ and cask are moved from the transporter to the cask-receiving pit. No change of material balance. See Stream IC-003. 


\subsubsection{C-4 Empty Transporter}

Decontaminate empty transporter as required before reassemble of shipping system. No material balance.

\subsubsection{C-5 Remove MCO From Cask}

There is no change in material balance. See Stream IC-003.

\subsubsection{C-6 Move MCO From Transport Cask to Weld Station in MHM}

The loaded MCO is moved from the transport cask to the weld station in the MCO handling machine (MHM). There is no change of material inside the MCO.

This is an input stream originating from cask transport Stream IC-003. The $392 \mathrm{MCOs}$ go directly to welding. The six-monitored MCOs are deleted from this stream. The stream mass is listed below: 
SNF-5741, Rev. 0

Table 7.2.2 Stream C-6

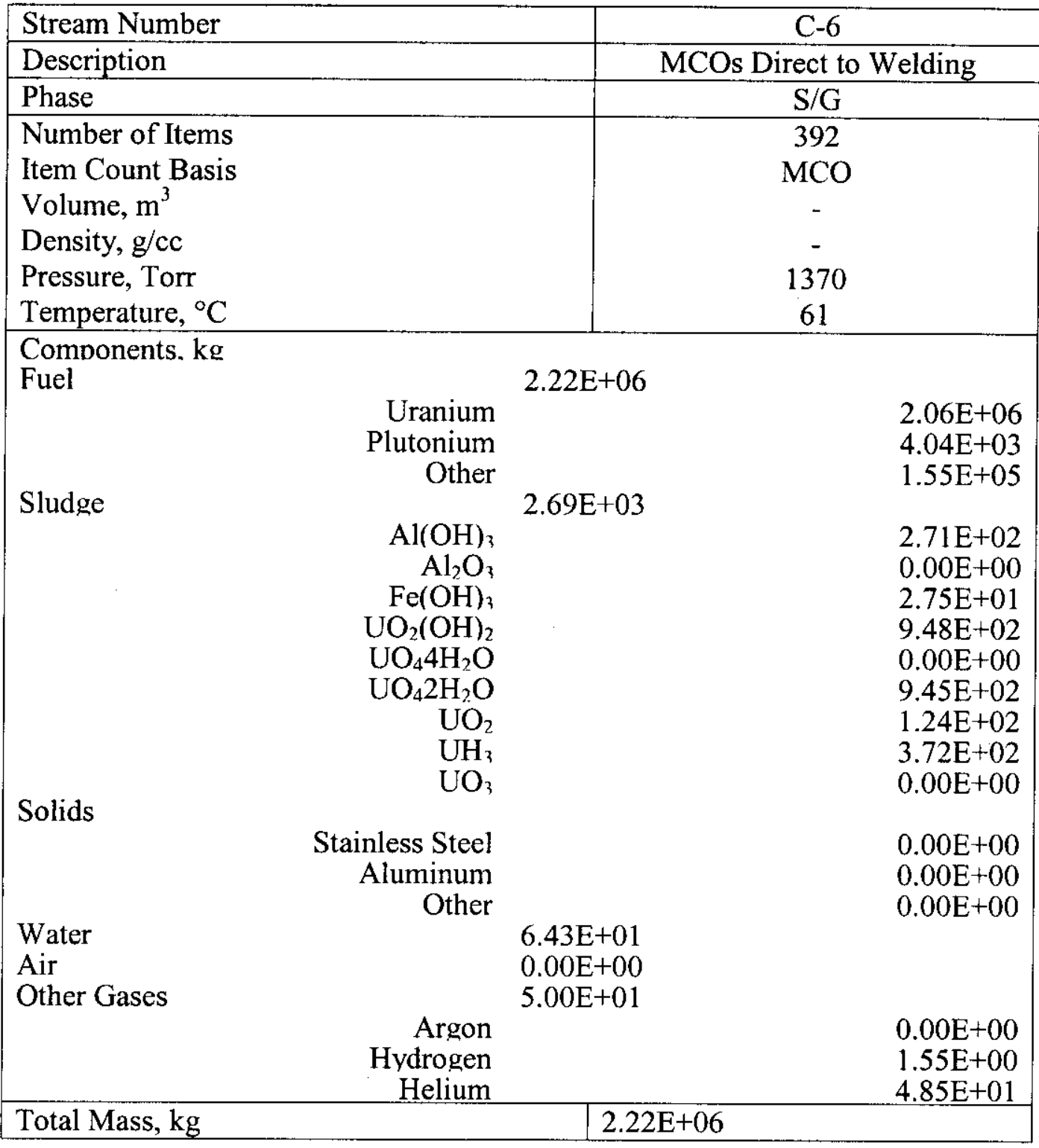




\subsubsection{C-7 Move Short Term Monitored MCO to Vault in MHM}

There are approximately six short term monitored MCOs over a two year period. The below material balance represents the six monitored MCOs at time zero.

Table 7.2.3 Stream C-7

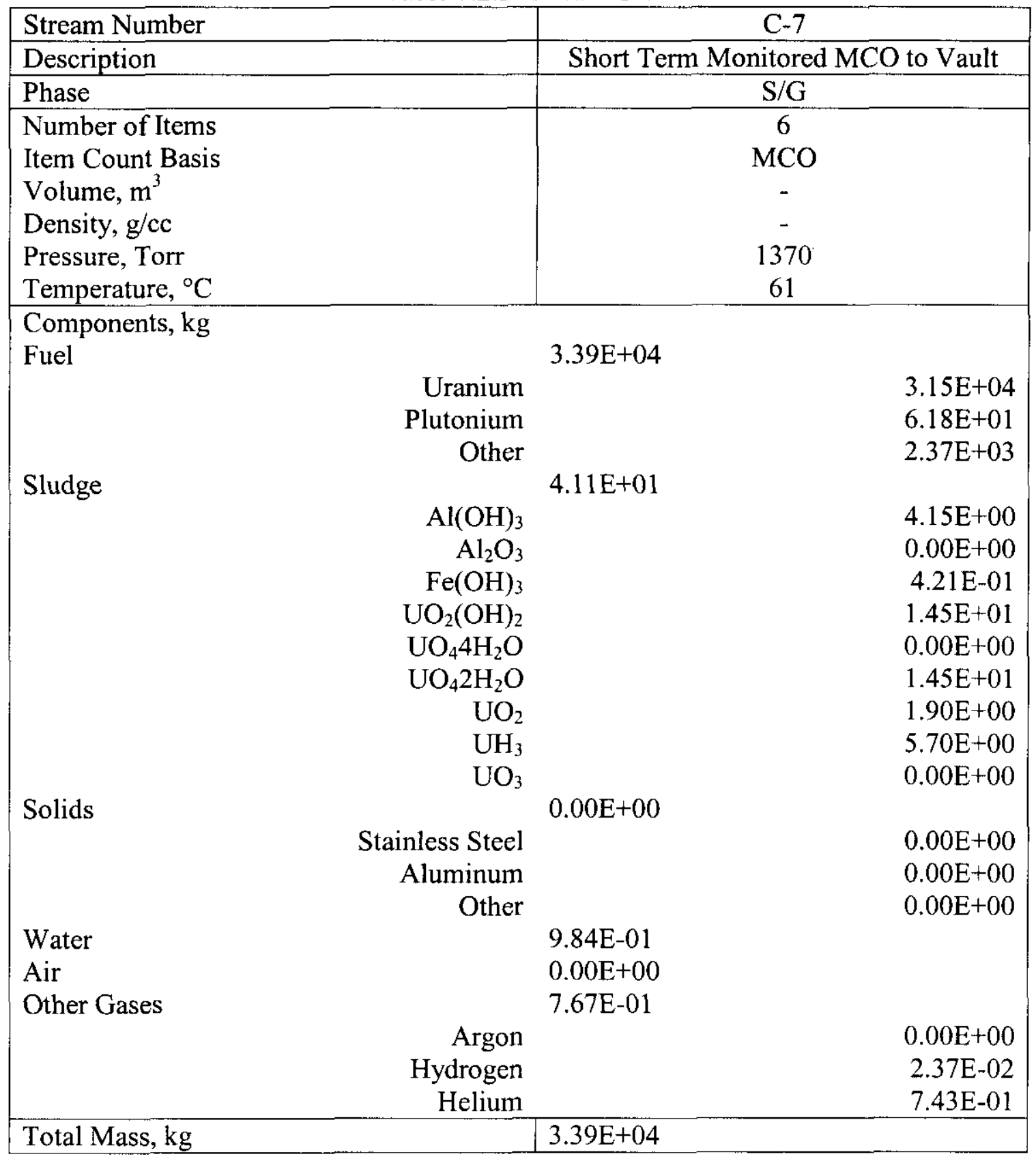




\subsubsection{C-8 Short Term Monitored MCO in Storage Tube}

Short term monitoring of the six preselected MCOs will have a small effect on the gas composition inside the MCOs. Four gas samples are taken from each monitored MCO/year at the sample/weld station over a two year period (private communication with G. D. Bazinet of Numatec Hanford Corporation). The lost helium and hydrogen is replaced with helium after each sampling (private communication with A. L. Pajunen of Fluor Hanford). See Stream C-7 for description of the six MCOs before monitoring and Stream C-12 for description of the six MCOs after two years of monitoring. The composition has been adjusted for radiolysis of hydrated oxides and refilling with helium to replace lost gas during sampling.

\subsubsection{C-9 Cask Receiving Pit Containment Tent Offgas}

The service pit containment tent is used for contamination control during recovery from off normal conditions such as high air pressure ( $>3 \mathrm{psig}$ ) inside the transport cask annulus (Reed and Pajunen 1999), smearable radionuclide contamination on the MCO top, and/or the cask lid wall exceeds maximum permissible levels of smearable contamination. The exhaust flowrate of the service tent is established by the design capacity of the filter system. Filter EF-005 has a design capacity of 1,000 scfm (Ellis and Watts 1999). The containment tent ventilation system would be operated for approximately four hours during a recovery operation based on the operation block sequence flow diagram (OBSFD) (H-2-123400, Rev. H).

\subsubsection{C-10 Cask Receiving Pit Containment Tent Replacement Filters}

Filter system EF-005 consists of one prefilter and one HEPA filter. The prefilter is 24 in $x 24$ in $x 2$ in and the HEPA filter size is 24 in $x 24$ in $x 11.5$ in (Ellis and Watts 1999). Assume the prefilter weights $12 \mathrm{lb}$ and the HEPA filter weighs $40 \mathrm{lb}$.

\subsubsection{C-11 Spent Cask Receiving Pit HEPA Filters}

Frequency of filter change is unknown at this time due to infrequent use of receiving pit containment tent.

\subsubsection{C-12 Short Term Monitored MCOs From Sampling to Storage Tube}

MCO composition has been adjusted to 2 years after receipt and backfilled with helium in order to replace estimated gas lost during gas sampling with the sample cart. Each of the six monitored MCOs is sampled a total of eight times over the two year monitoring period. 
SNF-5741, Rev. 0

Table 7.2.4 Stream C-12 (Before 100 PSI Pressure Sending/Readout Unit Replacement)

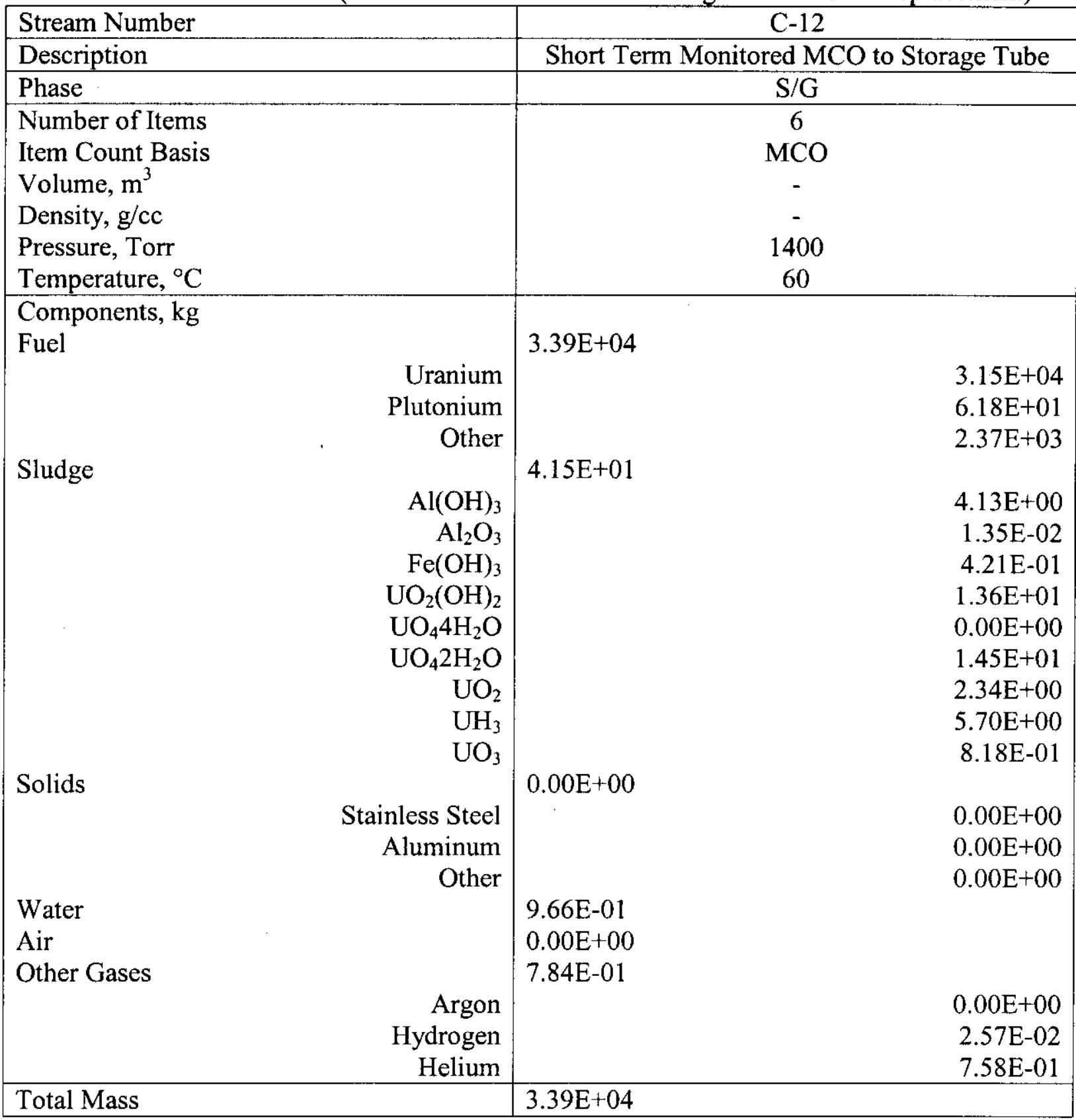


SNF-5741, Rev. 0

Table 7.2.5 Summary of Monitored MCO Sampling

\begin{tabular}{|c|c|c|c|c|c|c|c|c|}
\hline $\begin{array}{c}\text { Time } \\
\text { Yr }\end{array}$ & $\begin{array}{c}\text { Sample } \\
\mathrm{No}^{1}\end{array}$ & $\begin{array}{c}\text { Est MCO } \\
\text { press } \\
\text { psia }\end{array}$ & $\begin{array}{c}\text { Est } \\
\text { Temp } \\
{ }^{\circ} \mathrm{C}\end{array}$ & $\begin{array}{c}\text { Est } \\
\text { MCO } \mathrm{H}_{2} \\
\text { Vol \% }\end{array}$ & $\begin{array}{c}\text { Est MCO } \\
\text { He } \\
\text { Vol \% }\end{array}$ & $\begin{array}{c}\text { Est DP } \\
\text { MCO w } \\
\text { sampling } \\
\text { Psi }\end{array}$ & $\begin{array}{c}\text { Est } \mathrm{H}_{2} \text { lost } \\
\text { (sampling six } \\
\text { MCOs) } \\
\text { kg }\end{array}$ & $\begin{array}{c}\text { Est He lost } \\
\text { (sampling } \\
\text { six MCOs) } \\
\mathrm{kg}\end{array}$ \\
\hline 0.0 & - & 26.5 & 61 & 6.0 & 94.0 & - & - & - \\
\hline 0.25 & $1 \mathrm{~B}$ & 26.6 & 61 & 6.3 & 93.7 & 1.0 & $9.38 \mathrm{E}-04$ & $2.79 \mathrm{E}-02$ \\
\hline 0.25 & $1 \mathrm{~A}$ & 26.6 & 61 & 6.2 & 93.8 & - & - & - \\
\hline 0.5 & 2B & 26.7 & 61 & 6.3 & 93.7 & 1.0 & $9.39 \mathrm{E}-04$ & $2.79 \mathrm{E}-02$ \\
\hline 0.5 & $2 \mathrm{~A}$ & 26.7 & 61 & 6.1 & 93.9 & - & & \\
\hline 0.75 & $3 \mathrm{~B}$ & 26.8 & 61 & 6.3 & 93.7 & 1.0 & $9.39 \mathrm{E}-04$ & $2.79 \mathrm{E}-02$ \\
\hline 0.75 & $3 \mathrm{~A}$ & 26.8 & 61 & 6.1 & 93.9 & - & & \\
\hline 1.0 & $4 \mathrm{~B}$ & 26.8 & 61 & 6.3 & 93.7 & 1.0 & $9.39 \mathrm{E}-04$ & $2.79 \mathrm{E}-02$ \\
\hline 1.0 & $4 \mathrm{~A}$ & 26.8 & 61 & 6.1 & 93.9 & - & & \\
\hline Subtotal & & & & & & & $3.76 \mathrm{E}-03$ & $1.11 \mathrm{E}-01$ \\
\hline 1.25 & 5B & 26.8 & 60 & 6.4 & 93.6 & 1.0 & $9.42 \mathrm{E}-04$ & $2.80 \mathrm{E}-02$ \\
\hline 1.25 & $5 \mathrm{~A}$ & 26.8 & 60 & 6.1 & 93.9 & - & & \\
\hline 1.5 & $6 \mathrm{~B}$ & 26.8 & 60 & 6.3 & 93.7 & 1.0 & $9.42 \mathrm{E}-04$ & $2.80 \mathrm{E}-02$ \\
\hline 1.5 & $6 \mathrm{~A}$ & 26.8 & 60 & 6.1 & 93.9 & - & & \\
\hline 1.75 & $7 \mathrm{~B}$ & 27.0 & 60 & 6.4 & 93.6 & 1.0 & $9.56 \mathrm{E}-04$ & $2.80 \mathrm{E}-02$ \\
\hline 1.75 & $7 \mathrm{~A}$ & 27.0 & 60 & 6.2 & 93.8 & - & & \\
\hline 2.0 & $8 \mathrm{~B}$ & 27.0 & 60 & 6.4 & 93.6 & 1.0 & $9.56 \mathrm{E}-04$ & $2.80 \mathrm{E}-02$ \\
\hline 2.0 & $8 \mathrm{~A}$ & 27.0 & 60 & 6.3 & 93.7 & - & & \\
\hline Subtotal & - & - & - & - & - & - & $3.80 \mathrm{E}-03$ & $1.12 \mathrm{E}-01$ \\
\hline Grand \\
Total & - & - & - & - & - & - & $7.56 \mathrm{E}-03$ & $2.23 \mathrm{E}-01$ \\
\hline
\end{tabular}

1. $B=$ Before sampling, $A=$ After sampling with $\mathrm{He}$ refill to replace lost $\mathrm{H}_{2}$ and $\mathrm{He}$

Table 7.2.6 Estimated Data from Best Estimate Single Scrap Basket Case ${ }^{1}$

\begin{tabular}{|c|c|c|c|c|}
\hline $\begin{array}{c}\text { Time } \\
\text { Yr }\end{array}$ & $\begin{array}{c}\text { Est. MCO press } \\
\text { w/o sampling } \\
\text { psia }\end{array}$ & $\begin{array}{c}\text { Est. Avg. Fuel } \\
\text { Temp } \\
{ }^{\circ} \mathrm{C}\end{array}$ & $\begin{array}{c}\text { Est. } \mathrm{H}_{2} \\
\text { vol \% }\end{array}$ & $\begin{array}{c}\text { Est. He } \\
\text { vol \% }\end{array}$ \\
\hline 0.0 & 25.1 & 61 & 6.0 & 94.0 \\
\hline 0.5 & 25.1 & 61 & 6.6 & 93.4 \\
\hline 1.0 & 25.4 & 61 & 7.0 & 93.0 \\
\hline 1.5 & 25.6 & 60 & 7.5 & 92.5 \\
\hline 2.0 & 25.7 & 60 & 8.0 & 92.0 \\
\hline
\end{tabular}

1. Based on Duncan and Plys (1998) Page J-11

2. Based on scrap temperature

Mass balance calculations are listed below for Table 7.2.5. 


\section{SAMPLE 1B}

Determine mass of $\mathrm{H}_{2}$ in six monitored MCOs at $0.25 \mathrm{yr}$ and no sampling/refill of $\mathrm{MCO}$

$0.063=\mathrm{X} \mathrm{H}_{2} /\left(\mathrm{XH}_{2}+31.0 \mathrm{He}\right)$ based on Duncan and Plys (1998) and Pajunen (2000)

$\mathrm{X}=0.063 \times 31.0 / 0.937=2.084 \mathrm{gmol} \mathrm{H}_{2} / \mathrm{MCO}$

Pressure of MCO before sampling based on initial He fill of $31.0 \mathrm{gmol} / \mathrm{MCO}=\left(2.084 \mathrm{gmol} \mathrm{H}_{2}+\right.$ $31.0 \mathrm{gmol} \mathrm{He}=33.08 \mathrm{gmol} / \mathrm{MCO}) \times 0.082 \mathrm{~atm} \mathrm{~L} / \mathrm{gmol} \mathrm{K} \times 334 \mathrm{~K} /(500 \mathrm{~L} / \mathrm{MCO})=1.81 \mathrm{~atm}=$ 26.6 psia

$\mathrm{MCO} /$ sample cart pressure after pressurizing sample cart assuming no temperature effects $=$ $(\mathrm{P} 1 \mathrm{~V} 1+\mathrm{P} 2 \mathrm{~V} 2) /(\mathrm{V} 1+\mathrm{V} 2)=(26.6$ psia $\times 500 \mathrm{~L} \mathrm{MCO}+14.7$ psia $\times 46.4 \mathrm{~L}$ sample cart $) /(500 \mathrm{~L}+$ $46.4 \mathrm{~L})=25.6 \mathrm{psia}$

$\mathrm{DP}=26.6 \mathrm{psia}-25.6 \mathrm{psia}=1 \mathrm{psia}$

Mass $\mathrm{H}_{2}$ lost from six monitored MCOs after sampling at $0.25 \mathrm{yr}=6 \times \mathrm{DP} \times \mathrm{V} \times 0.063 \mathrm{H}_{2} / \mathrm{RT}=$ 6 MCOs x 1.0 psia x $500 \mathrm{~L} / \mathrm{MCO} \times 0.063 \mathrm{H}_{2} /(14.7 \mathrm{psia} / \mathrm{atm} \times 0.0820 \mathrm{~L}$ atm $/ \mathrm{gmol} \mathrm{K} \times 334 \mathrm{~K}) \times$ $0.001 \mathrm{~kg} / \mathrm{g}=4.69 \mathrm{E}-04 \mathrm{~kg} \mathrm{~mol}=9.38 \mathrm{E}-04 \mathrm{~kg}$

Mass He lost from six monitored MCOs after sampling at $0.25 \mathrm{yr}=6 \mathrm{MCOs} \times \mathrm{DP} \times \mathrm{V} \times 0.937$ $\mathrm{He} / \mathrm{RT}=6 \mathrm{MCOs} \times 1.0 \mathrm{psia} \times 500 \mathrm{~L} / \mathrm{MCO} \times 0.937 \mathrm{He} /(14.7 \mathrm{psia} / \mathrm{atm} \times 0.0820 \mathrm{~L} \mathrm{~atm} / \mathrm{gmol} \mathrm{K} \mathrm{x}$ $334 \mathrm{~K}) \times 0.001 \mathrm{~kg} / \mathrm{g}=6.98 \mathrm{E}-03 \mathrm{~kg} \mathrm{~mol}=2.79 \mathrm{E}-02 \mathbf{~ k g}$

\section{SAMPLE 1A}

Mass $\mathrm{H}_{2}$ remaining inside six monitored MCOs after sampling at $0.25 \mathrm{yr}=2.084 \mathrm{gmol} / \mathrm{MCO} \mathrm{x}$ $6 \mathrm{MCOs} \times 0.001 \mathrm{~kg} / \mathrm{g}-4.69 \mathrm{E}-04 \mathrm{~kg} \mathrm{~mol}$ lost $=1.203 \mathrm{E}-02 \mathrm{~kg} \mathrm{~mol}=2.40 \mathrm{E}-02 \mathrm{~kg}$

Mass He remaining inside six monitored MCOs after sampling at $0.25 \mathrm{yr}$ and before refill $=$ $3.10 \mathrm{E}-02 \mathrm{~kg} \mathrm{~mol} / \mathrm{MCO} \times 6 \mathrm{MCOs}-6.98 \mathrm{E}-03 \mathrm{~kg} \mathrm{~mol}$ lost $=1.790 \mathrm{E}-01 \mathrm{~kg} \mathrm{~mol}=7.161 \mathrm{E}-01 \mathrm{~kg}$

Pressure in reinerted/monitored MCOs at $0.25 \mathrm{yr}=\mathrm{nRT} / \mathrm{V}=\left(1.203 \mathrm{E}-02 \mathrm{~kg}\right.$ mole $\mathrm{H}_{2}$ remaining + $4.69 \mathrm{E}-04 \mathrm{~kg} \mathrm{~mol}$ from $\mathrm{H}_{2}$ lost $+6.985 \mathrm{E}-03 \mathrm{~kg}$ mol He lost $+1.790 \mathrm{E}-01 \mathrm{~kg}$ mol He remaining $=$ $1.985 \mathrm{E}-01) \times 0.0820 \mathrm{~atm} \mathrm{~L} / \mathrm{gmol} \mathrm{K} \times 334 \mathrm{~K}) \times 1000 \mathrm{~g} / \mathrm{kg} /(500 \mathrm{~L} / \mathrm{MCO} \times 6 \mathrm{MCOs})=1.81 \mathrm{~atm}=$ 26.6 psia

Mass of He inside six monitored MCOs after reinert at $0.25 \mathrm{yr}=4.69 \mathrm{E}-04 \mathrm{~kg} \mathrm{~mol} \mathrm{H} \mathrm{H}_{2}$ lost + $6.985 \mathrm{E}-03 \mathrm{~kg}$ mol He lost $+1.790 \mathrm{E}-01 \mathrm{~kg}$ mol remaining $=1.864 \mathrm{E}-01 \mathrm{~kg}$ mol

Concentration of $\mathrm{H}_{2}$ in reinerted $\mathrm{MCO}$ after sampling at $0.25 \mathrm{yr}=1.203 \mathrm{E}-02 \mathrm{~kg} \mathrm{~mol} \mathrm{x}$ $100 /(1.864 \mathrm{E}-01 \mathrm{~kg} \mathrm{~mol} \mathrm{He}+1.203 \mathrm{E}-02 \mathrm{~kg} \mathrm{~mol} \mathrm{H}=1.985 \mathrm{E}-01 \mathrm{~kg} \mathrm{~mol})=\mathbf{6 . 2} \mathrm{vol} \%$

Concentration of $\mathrm{He}$ in reinerted $\mathrm{MCO}$ after sampling at $0.25 \mathrm{yr}=100-6.2=\mathbf{9 3 . 8} \%$ 
SNF-5741, Rev. 0

\section{SAMPLE 2B}

Determine mass of $\mathrm{H}_{2}$ in six monitored MCOs at $0.5 \mathrm{yr}$ and no sampling/He reinerting

$0.066=\mathrm{X} \mathrm{H}_{2} /\left(\mathrm{XH}_{2}+31.0 \mathrm{He}\right)$ based on Duncan and Plys (1998) and Pajunen (2000)

$\mathrm{X}=0.066 \times 31.0 / 0.937=2.183 \mathrm{E}+00 \mathrm{gmol} \mathrm{H}_{2} / \mathrm{MCO}$

Amount $\mathrm{H}_{2}$ in six monitored MCOs before sampling at $0.5 \mathrm{yr}$ (sampling and after reinert at 0.25 $\mathrm{yr})=2.183 \mathrm{gmol} / \mathrm{MCO} \times 6 \mathrm{MCOs} \times 0.001 \mathrm{~kg} / \mathrm{g}-4.69 \mathrm{E}-04 \mathrm{~kg}$ mol lost at $0.25 \mathrm{yr}=1.263 \mathrm{E}-02 \mathrm{~kg}$ mol

Concentration of hydrogen at $0.5 \mathrm{yr}$ before sampling $=1.263 \mathrm{E}-02 \mathrm{~kg} \mathrm{~mol} \mathrm{H} \mathrm{H}_{2} \times 100 /(1.263 \mathrm{E}-02$ $\mathrm{kg} \mathrm{mol} \mathrm{H}+1.864 \mathrm{E}-01 \mathrm{~kg} \mathrm{~mol} \mathrm{He}=1.990 \mathrm{E}-01)=6.3 \%$

Concentration of $\mathrm{He}$ at $0.5 \mathrm{yr}$ before sampling $=100-6.3=93.7 \%$

MCO Pressure at $0.5 \mathrm{yr}$ before sampling $=1.990 \mathrm{E}-01 \mathrm{~kg} \mathrm{~mol} \mathrm{x} 0.0820 \mathrm{~L} \mathrm{~atm} / \mathrm{gmol} \mathrm{K} \mathrm{x} 334 \mathrm{~K} \mathrm{x}$ $1000 \mathrm{~g} / \mathrm{kg} /(500 \mathrm{~L} / \mathrm{MCO} \times 6 \mathrm{MCOs})=1.817 \mathrm{~atm}=\mathbf{2 6 . 7} \mathbf{p s i a}$

$\mathrm{MCO} /$ sample cart pressure after pressurizing sample cart assuming no temperature effects = $(\mathrm{P} 1 \mathrm{~V} 1+\mathrm{P} 2 \mathrm{~V} 2) /(\mathrm{V} 1+\mathrm{V} 2)=(26.7 \mathrm{psia} \times 500 \mathrm{~L}+14.7 \mathrm{psia} \times 46.4 \mathrm{~L}) /(500 \mathrm{~L}+46.4 \mathrm{~L})=25.7 \mathrm{psia}$

\section{$\mathrm{DP}=26.7 \mathrm{psia}-25.7 \mathrm{psia}=\mathbf{1 . 0}$ psia}

Mass $\mathrm{H}_{2}$ lost from six monitored MCOs after sampling at $0.5 \mathrm{yr}=6 \mathrm{MCOs} \times \mathrm{DP} \times \mathrm{V} \times 0.063$ $\mathrm{H}_{2} / \mathrm{RT}=6 \mathrm{MCOs} \times 1.0 \mathrm{psia} \times 500 \mathrm{~L} / \mathrm{MCO} \times 0.063 /(14.7 \mathrm{psia} / \mathrm{atm} \times 0.0820 \mathrm{~L} \mathrm{~atm} / \mathrm{gmol} \mathrm{K} \times 334$ K) $\times 0.001 \mathrm{~kg} / \mathrm{g}=4.69 \mathrm{E}-04 \mathrm{~kg} \mathrm{~mol}=9.39 \mathrm{E}-04 \mathrm{~kg}$

Mass He lost from six monitored MCOs after sampling at $0.5 \mathrm{yr}=6 \mathrm{MCOs} \times \mathrm{DP} \times \mathrm{V} \mathrm{x} 0.937$ $\mathrm{He} / \mathrm{RT}=6 \mathrm{MCOs} \times 1.0 \mathrm{psia} \times 500 \mathrm{~L} / \mathrm{MCO} \times 0.937 \mathrm{He} /(14.7 \mathrm{psia} / \mathrm{atm} \times 0.0820 \mathrm{~L} \mathrm{~atm} / \mathrm{gmol} \mathrm{K} \mathrm{x}$ $334 \mathrm{~K}) \times 0.001 \mathrm{~kg} / \mathrm{g}=6.982 \mathrm{E}-03 \mathrm{~kg} \mathrm{~mol}=\mathbf{2 . 7 9 E - 0 2 ~} \mathbf{~ k g}$

\section{SAMPLE 2A}

Mass $\mathrm{H}_{2}$ remaining in six monitored MCOs after sampling at $0.5 \mathrm{yr}=1.263 \mathrm{E}-02 \mathrm{~kg} \mathrm{~mol}-$ $4.69 \mathrm{E}-04 \mathrm{~kg}$ mol lost at $0.5 \mathrm{yr}=1.216 \mathrm{E}-02 \mathrm{~kg} \mathrm{~mol}=2.43 \mathrm{E}-02 \mathrm{~kg}$

Mass He remaining in six monitored MCOs after sampling at $0.5 \mathrm{yr}=1.864 \mathrm{E}-01 \mathrm{~kg} \mathrm{~mol}-$ $6.982 \mathrm{E}-03 \mathrm{~kg}$ mol lost at $0.5 \mathrm{yr}=1.794 \mathrm{E}-01 \mathrm{~kg} \mathrm{~mol}=7.18 \mathrm{E}-01 \mathrm{~kg}$

Pressure in reinerted $/$ monitored MCOs at $0.5 \mathrm{yr}=\mathrm{nRT} / \mathrm{V}=(1.216 \mathrm{E}-02 \mathrm{~kg} \mathrm{~mol} \mathrm{H}$ remaining + 4.69E-04 kg mol from $\mathrm{H}_{2}$ lost $+6.98 \mathrm{E}-03 \mathrm{~kg} \mathrm{~mol} \mathrm{He} \mathrm{lost} \mathrm{+} \mathrm{1.794E-01} \mathrm{kg} \mathrm{mol} \mathrm{He} \mathrm{remaining} \mathrm{=}$ $1.990 \mathrm{E}-01) \times 0.0820 \mathrm{~atm} \mathrm{~L} / \mathrm{gmol} \mathrm{K} \times 334 \mathrm{~K}) \times 1000 \mathrm{~g} / \mathrm{kg} /(500 \mathrm{~L} / \mathrm{MCO} \times 6 \mathrm{MCOs})=1.82 \mathrm{~atm}=$ 26.7 psia 
Mass of He in six monitored MCOs after reinert at $0.5 \mathrm{yr}=1.794 \mathrm{E}-01 \mathrm{~kg} \mathrm{~mol} \mathrm{remaining} \mathrm{+}$ $6.982 \mathrm{E}-03 \mathrm{~kg} \mathrm{~mol} \mathrm{He}$ lost $+4.69 \mathrm{E}-04 \mathrm{~kg} \mathrm{~mol} \mathrm{H} \mathrm{H}_{2} \mathrm{lost}=1.868 \mathrm{E}-01 \mathrm{~kg} \mathrm{~mol}$

Concentration of $\mathrm{H}_{2}$ in $\mathrm{MCO}$ at $0.5 \mathrm{yr}$ after reinert $=1.216 \mathrm{E}-02 \mathrm{~kg} \mathrm{~mol} \mathrm{H}$ x $100 /(1.216 \mathrm{E}-02 \mathrm{~kg}$ $\mathrm{mol} \mathrm{H}+1.868 \mathrm{E}-01 \mathrm{~kg} \mathrm{~mol} \mathrm{He}=1.990 \mathrm{E}-01)=6.1 \mathrm{vol} \%$

Concentration of $\mathrm{He}=100-6.1=93.9$ vol $\%$

\section{SAMPLE 3B}

Determine mass of $\mathrm{H}_{2}$ in six monitored MCOs at $0.75 \mathrm{yr}$ and no sampling/He reinerting $0.068 \mathrm{H}_{2}=\mathrm{X} \mathrm{H}_{2} /\left(\mathrm{X} \mathrm{H}_{2}+31.0 \mathrm{He}\right)$ based on Duncan and Plys (1998) and Pajunen (2000) $\mathrm{X}=0.068 \times 31.0 / 0.932=2.262 \mathrm{E}+00$ gmol $\mathrm{H}_{2} / \mathrm{MCO}$ w/o sampling and reinerting

Amount $\mathrm{H}_{2}$ remaining in six monitored MCOs before sampling at $0.75 \mathrm{yr}$ (sampling and refill at $0.5 \mathrm{yr})=2.262 \mathrm{E}-03 \mathrm{gmol} / \mathrm{MCO} \times 6 \mathrm{MCOs} \times 0.001 \mathrm{~kg} / \mathrm{g}-4.69 \mathrm{E}-04 \mathrm{~kg}$ mol lost at $0.25 \mathrm{yr}-$ $4.69 \mathrm{E}-04 \mathrm{~kg} \mathrm{~mol}$ at $0.5 \mathrm{yr}=1.263 \mathrm{E}-02 \mathrm{~kg} \mathrm{~mol}$

Concentration of hydrogen at $0.75 \mathrm{yr}$ before sampling $=1.263 \mathrm{E}-02 \mathrm{~kg} \mathrm{~mol} \mathrm{H} \mathrm{H}_{2} \times 100 /(1.263 \mathrm{E}-02$ $\mathrm{kg} \mathrm{mol} \mathrm{H}+1.868 \mathrm{E}-01 \mathrm{~kg} \mathrm{~mol} \mathrm{He}=1.994 \mathrm{E}-01)=6.3 \%$

Concentration of $\mathrm{He}$ at $0.75 \mathrm{yr}$ before sampling $=100-6.3=\mathbf{9 3 . 7} \%$

MCO Pressure at $0.75 \mathrm{yr}$ before sampling $=1.994 \mathrm{E}-01 \mathrm{~kg} \mathrm{~mol} \mathrm{x} 0.0820 \mathrm{~L}$ atm $/ \mathrm{gmol} \mathrm{K} \mathrm{x} 334 \mathrm{~K} \mathrm{x}$ $1000 \mathrm{~g} / \mathrm{kg} /(500 \mathrm{~L} / \mathrm{MCO} \times 6 \mathrm{MCOs})=1.82 \mathrm{~atm}=\mathbf{2 6 . 8} \mathbf{~ p s i a}$

$\mathrm{MCO} /$ sample cart pressure after pressurizing sample cart assuming no temperature effects = $(\mathrm{P} 1 \mathrm{~V} 1+\mathrm{P} 2 \mathrm{~V} 2) /(\mathrm{V} 1+\mathrm{V} 2)=(26.8 \mathrm{psia} \times 500 \mathrm{~L}+14.7 \mathrm{psia} \times 46.4 \mathrm{~L}) /(500 \mathrm{~L}+46.4 \mathrm{~L})=25.8 \mathrm{psia}$

$\mathrm{DP}=26.8 \mathrm{psia}-25.8 \mathrm{psia}=1.0 \mathrm{psia}$

Mass $\mathrm{H}_{2}$ lost from six monitored MCOs after sampling at $0.75 \mathrm{yr}=6 \mathrm{MCO} \times \mathrm{DP} \times \mathrm{V} \times 0.063$ $\mathrm{H}_{2} / \mathrm{RT}=6 \mathrm{MCOs} \times 1.0 \mathrm{psia} \times 500 \mathrm{~L} / \mathrm{MCO} \times 0.063 /(14.7 \mathrm{psia} / \mathrm{atm} \times 0.0820 \mathrm{~L} \mathrm{~atm} / \mathrm{gmol} \mathrm{K} \times 334$ $\mathrm{K}) \times 0.001 \mathrm{~kg} / \mathrm{g}=4.69 \mathrm{E}-04 \mathrm{~kg} \mathrm{~mol}=9.39 \mathrm{E}-04 \mathrm{~kg}$

Mass He lost from six monitored MCOs after sampling at $0.75 \mathrm{yr}=6 \mathrm{MCOs} \times \mathrm{DP} \times \mathrm{V} \times 0.937$ $\mathrm{He} / \mathrm{RT}=6 \mathrm{MCOs} \times 1.0 \mathrm{psia} \times 500 \mathrm{~L} \times 0.937 \mathrm{He} /(14.7 \mathrm{psia} / \mathrm{atm} \times 0.0820 \mathrm{~L}$ atm/gmol K $\times 334 \mathrm{~K})$ $x 0.001 \mathrm{~kg} / \mathrm{g}=6.98 \mathrm{E}-03 \mathrm{~kg} \mathrm{~mol}=\mathbf{2 . 7 9 E}-\mathbf{0 2} \mathbf{~ k g}$

\section{SAMPLE 3A}

Mass $\mathrm{H}_{2}$ remaining in six monitored MCOs after sampling at $0.75 \mathrm{yr}=1.263 \mathrm{E}-02 \mathrm{~kg} \mathrm{~mol}-$ $4.69 \mathrm{E}-04 \mathrm{~kg} \mathrm{~mol}$ at $0.75 \mathrm{yr}=1.216 \mathrm{E}-02 \mathrm{~kg} \mathrm{~mol}=2.43 \mathrm{E}-02 \mathrm{~kg}$ 
Mass He remaining in six monitored MCOs after sampling at $0.75 \mathrm{yr}$ and before reinerting = $1.868 \mathrm{E}-01 \mathrm{~kg} \mathrm{~mol}-6.98 \mathrm{E}-03 \mathrm{~kg} \mathrm{~mol}=1.798 \mathrm{E}-01 \mathrm{~kg} \mathrm{~mol}$

Mass He in reinerted MCOs after sampling and reinert at $0.75 \mathrm{yr}=1.798 \mathrm{E}-01 \mathrm{~kg}$ mol remaining $+6.98 \mathrm{E}-03 \mathrm{~kg} \mathrm{~mol} \mathrm{He} \mathrm{lost}+4.69 \mathrm{E}-04 \mathrm{~kg} \mathrm{~mol} \mathrm{H} 2$ lost $=1.872 \mathrm{E}-01 \mathrm{~kg} \mathrm{~mol}$

Concentration of $\mathrm{H}_{2}$ in $\mathrm{MCO}$ at $0.75 \mathrm{yr}$ after reinerting $=1.216 \mathrm{E}-02 \mathrm{~kg} \mathrm{~mol} \mathrm{H} \mathrm{H}_{2} \times 100 /(1.216 \mathrm{E}-02$ $\mathrm{kg} \mathrm{mol} \mathrm{H}+1.872 \mathrm{E}-01 \mathrm{~kg} \mathrm{~mol} \mathrm{He})=\mathbf{6 . 1}$ vol \%

Concentration of $\mathrm{He}=100-6.1=93.9$ vol \%

Pressure in refilled/monitored MCOs at $0.75 \mathrm{yr}=\mathrm{nRT} / \mathrm{V}=\left(1.216 \mathrm{E}-02 \mathrm{~kg} \mathrm{~mol} \mathrm{H} \mathrm{H}_{2}+1.872 \mathrm{E}-01\right.$ $\mathrm{kg} \mathrm{mol} \mathrm{He}=1.993 \mathrm{E}-01) \times 0.0820 \mathrm{~atm} \mathrm{~L} / \mathrm{gmol} \mathrm{K} \times 334 \mathrm{~K}) \times 1000 \mathrm{~g} / \mathrm{kg} /(500 \mathrm{~L} / \mathrm{MCO} \times 6 \mathrm{MCOs})$ $=1.82 \mathrm{~atm}=26.8 \mathrm{psia}$

\section{SAMPLE 4B}

Determine mass of $\mathrm{H}_{2}$ in six monitored MCOs at $1.0 \mathrm{yr}$ and no sampling/He reinerting $0.070 \mathrm{H}_{2}=\mathrm{X} \mathrm{H}_{2} /\left(\mathrm{X} \mathrm{H}_{2}+31.0 \mathrm{He}\right)$ based on Duncan and Plys (1998) and Pajunen (2000) $\mathrm{X}=0.070 \times 31.0 / 0.930=2.333 \mathrm{E}+00 \mathrm{gmol} \mathrm{H}_{2} / \mathrm{MCO} \mathrm{w} / \mathrm{o} \mathrm{sampling}$ and reinerting

Amount $\mathrm{H}_{2}$ remaining in six monitored MCOs before sampling at $1.0 \mathrm{yr}$ (sampling and reinert at $0.75 \mathrm{yr})=2.333 \mathrm{gmol} / \mathrm{MCO} \times 6 \mathrm{MCOs} \times 0.001 \mathrm{~kg} / \mathrm{g}-4.69 \mathrm{E}-04 \mathrm{~kg}$ mol lost at $0.25 \mathrm{yr}-4.69 \mathrm{E}-04$ $\mathrm{kg} \mathrm{mol} \mathrm{lost} \mathrm{at} 0.5 \mathrm{yr}-4.69 \mathrm{E}-04 \mathrm{~kg} \mathrm{~mol}$ lost at $0.75 \mathrm{yr}=1.259 \mathrm{E}-02 \mathrm{~kg} \mathrm{~mol}$

Concentration of hydrogen at $1.0 \mathrm{yr}$ and before sampling $=1.259 \mathrm{E}-02 \mathrm{~kg} \mathrm{~mol} \mathrm{H} \mathrm{H}_{2} \times 100 /(1.259 \mathrm{E}-$ $02 \mathrm{~kg} \mathrm{~mol} \mathrm{H}+1.872 \mathrm{E}-01 \mathrm{~kg} \mathrm{~mol} \mathrm{He}=1.998 \mathrm{E}-01)=\mathbf{6 . 3} \%$

Concentration of $\mathrm{He}$ at $0.75 \mathrm{yr}$ before sampling $=100-6.3=93.7 \%$

MCO pressure at $1.0 \mathrm{yr}$ before sampling $=1.998 \mathrm{E}-01 \mathrm{~kg} \mathrm{~mol} \mathrm{x} 0.0820 \mathrm{~L}$ atm $/ \mathrm{gmol} \mathrm{K} \mathrm{x} 334 \mathrm{~K} \mathrm{x}$ $1000 \mathrm{~g} / \mathrm{kg} /(500 \mathrm{~L} / \mathrm{MCO} \times 6 \mathrm{MCOs})=1.82 \mathrm{~atm}=\mathbf{2 6 . 8} \mathbf{p s i a}$

$\mathrm{MCO} /$ sample cart pressure after pressurizing sample cart $=(\mathrm{P} 1 \mathrm{~V} 1+\mathrm{P} 2 \mathrm{~V} 2) /(\mathrm{V} 1+\mathrm{V} 2)=(26.8$ psia $\times 500 \mathrm{~L}+14.7$ psia $\times 46.4 \mathrm{~L}) /(500 \mathrm{~L}+46.4 \mathrm{~L})=25.8$ psia

$\mathrm{DP}=26.8 \mathrm{psia}-25.8 \mathrm{psia}=\mathbf{1 . 0} \mathbf{p s i a}$

Mass $\mathrm{H}_{2}$ lost from six monitored MCOs after sampling at $1.0 \mathrm{yr}=6 \mathrm{MCOs} \times \mathrm{DP} \times \mathrm{V} \times 0.063 \mathrm{x}$ $\mathrm{H}_{2} / \mathrm{RT}=6 \mathrm{MCOs} \times 1.0 \mathrm{psia} \times 500 \mathrm{~L} / \mathrm{MCO} \times 0.063 /(14.7 \mathrm{psia} / \mathrm{atm} \times 0.0820 \mathrm{~L} \mathrm{~atm} / \mathrm{gmol} \mathrm{K} \times 334$ K) $\times 0.001 \mathrm{~kg} / \mathrm{g}=4.69 \mathrm{E}-04 \mathrm{~kg} \mathrm{~mol}=9.39 \mathrm{E}-04 \mathrm{~kg}$ 
Mass He lost from six monitored MCOs after sampling at $1.0 \mathrm{yr}=6 \times \mathrm{DP} \times \mathrm{V} \times 0.937 \mathrm{He} / \mathrm{RT}=$ $6 \mathrm{MCOs} \times 1.0 \mathrm{psia} \times 500 \mathrm{~L} / \mathrm{MCO} \times 0.937 \mathrm{He} /(14.7 \mathrm{psia} / \mathrm{atm} \times 0.0820 \mathrm{~L}$ atm $/ \mathrm{gmol} \mathrm{K} \times 334 \mathrm{~K}) \times$ $0.001 \mathrm{~kg} / \mathrm{g}=6.98 \mathrm{E}-03 \mathrm{~kg} \mathrm{~mol}=2.79 \mathrm{E}-02 \mathrm{~kg}$

\section{SAMPLE 4A}

Mass $\mathrm{H}_{2}$ remaining in six monitored MCOs after sampling at $1.0 \mathrm{yr}=1.259 \mathrm{E}-02 \mathrm{~kg} \mathrm{~mol}-$ $4.69 \mathrm{E}-04 \mathrm{~kg} \mathrm{~mol}$ at $1.0 \mathrm{yr}=1.212 \mathrm{E}-02 \mathrm{~kg} \mathrm{~mol}=2.42 \mathrm{E}-02 \mathrm{~kg}$

Mass He remaining in six monitored MCOs after sampling at $1.0 \mathrm{yr}$ and before reinerting $=$ $1.872 \mathrm{E}-01 \mathrm{~kg} \mathrm{~mol}-6.98 \mathrm{E}-03 \mathrm{~kg} \mathrm{~mol}=1.802 \mathrm{E}-01 \mathrm{~kg} \mathrm{~mol}$

Mass $\mathrm{He}$ in reinerted MCOs after sampling and reinerting at $1.0 \mathrm{yr}=1.802 \mathrm{E}-01 \mathrm{~kg} \mathrm{~mol}$ remaining $+6.98 \mathrm{E}-03 \mathrm{~kg} \mathrm{~mol} \mathrm{He}$ lost $+4.69 \mathrm{E}-04 \mathrm{~kg} \mathrm{~mol} \mathrm{H}$ lost $=1.876 \mathrm{E}-01 \mathrm{~kg} \mathrm{~mol}$

Concentration of $\mathrm{H}_{2}$ in $\mathrm{MCO}$ at $1.0 \mathrm{yr}$ and after He reinerting $=1.212 \mathrm{E}-02 \mathrm{~kg} \mathrm{~mol} \mathrm{H} \mathrm{H}_{2}$ $100 /(1.212 \mathrm{E}-02 \mathrm{~kg} \mathrm{~mol} \mathrm{H}+1.876 \mathrm{E}-01 \mathrm{~kg} \mathrm{~mol} \mathrm{He}=1.997 \mathrm{E}-01 \mathrm{~kg} \mathrm{~mol})=\mathbf{6 . 1} \mathrm{vol} \%$

Concentration of $\mathrm{He}$ in $\mathrm{MCO}$ at $1 \mathrm{yr}$ and after He reinert $=100-6.1=93.9 \mathrm{vol} \%$

Pressure inside MCOs at $1.0 \mathrm{yr}$ and after He reinert $=1.997 \mathrm{E}-01 \mathrm{~kg} \mathrm{~mol} \mathrm{x} 0.0820 \mathrm{~atm} \mathrm{~L} / \mathrm{gmol} \mathrm{K}$ $\times 334 \mathrm{~K}) \times 1000 \mathrm{~g} / \mathrm{kg} /(500 \mathrm{~L} / \mathrm{MCO} \times 6 \mathrm{MCOs})=1.82 \mathrm{~atm}=\mathbf{2 6 . 8}$ psia

\section{SAMPLE 5B}

Determine mass of $\mathrm{H}_{2}$ in six monitored MCOs at $1.25 \mathrm{yr}$ and no sampling/He reinerting

$0.073 \mathrm{H}_{2}=\mathrm{X} \mathrm{H}_{2} /\left(\mathrm{X} \mathrm{H}_{2}+31.0 \mathrm{He}\right)$ based on Duncan and Plys (1998) and Pajunen (2000)

$\mathrm{X} \mathrm{H}_{2}=0.073 \times 31.0 / 0.927=2.441 \mathrm{E}+00 \mathrm{gmol} \mathrm{H}_{2} / \mathrm{MCO}$ w/o sampling and reinerting

Amount of $\mathrm{H}_{2}$ in six monitored MCOs before sampling at $1.25 \mathrm{yr}$ (sampling and reinerting at 1.0 $\mathrm{yr})=2.441 \mathrm{gmol} / \mathrm{MCO} \times 6 \mathrm{MCOs} \times 0.001 \mathrm{~kg} / \mathrm{g}-4.69 \mathrm{E}-04 \mathrm{~kg}$ mol$/ \mathrm{sample}$ event $\mathrm{x} 4$ sample events $=1.277 \mathrm{E}-02 \mathrm{~kg} \mathrm{~mol}$

Concentration of $\mathrm{H}_{2}$ at $1.25 \mathrm{yr}$ and before sampling $=1.277 \mathrm{E}-02 \mathrm{~kg} \mathrm{~mol} \mathrm{x} 100 /(1.277 \mathrm{E}-02 \mathrm{~kg}$ $\left.\mathrm{mol} \mathrm{H}_{2}+0.1876 \mathrm{~kg} \mathrm{~mol} \mathrm{He}=2.00 \mathrm{E}-01\right)=6.4 \mathrm{vol} \%$

Concentration of $\mathrm{He}$ at $1.25 \mathrm{yr}$ and before sampling $=100-6.4=93.7 \mathrm{vol} \%$

MCO pressure at $1.25 \mathrm{yr}$ before sampling $=2.00 \mathrm{E}-01 \times 0.0820 \mathrm{~atm} \mathrm{~L} / \mathrm{gmol} \mathrm{K} \times 333 \mathrm{~K}) \times 1000$ $\mathrm{g} / \mathrm{kg} /(500 \mathrm{~L} / \mathrm{MCO} \times 6 \mathrm{MCOs})=1.82 \mathrm{~atm}=\mathbf{2 6 . 8} \mathbf{p s i a}$

$\mathrm{MCO} /$ sample cart pressure after pressurizing sample cart $=(\mathrm{P} 1 \mathrm{~V} 1+\mathrm{P} 2 \mathrm{~V} 2) /(\mathrm{V} 1+\mathrm{V} 2)=(26.8$ psia $\times 500 \mathrm{~L}+14.7$ psia $\times 46.4 \mathrm{~L}) /(500 \mathrm{~L}+46.4 \mathrm{~L})=25.8$ psia 
$\mathrm{DP}=26.8 \mathrm{psia}-25.8 \mathrm{psia}=1$ psia

Mass $\mathrm{H}_{2}$ lost from six monitored MCOs after sampling at $1.25 \mathrm{yr}=6 \mathrm{MCOs} \times \mathrm{DP} \times \mathrm{V} \times 0.064$ $\mathrm{H}_{2} / \mathrm{RT}=6 \mathrm{MCOs} \times 1.0 \mathrm{psia} \times 500 \mathrm{~L} / \mathrm{MCO} \times 0.063 \mathrm{H}_{2} /(14.7 \mathrm{psia} / \mathrm{atm} \times 0.082 \mathrm{~L} \mathrm{~atm} / \mathrm{gmol} \mathrm{K} \mathrm{x}$ $333 \mathrm{~K}) \times 0.001 \mathrm{~kg} / \mathrm{g}=4.71 \mathrm{E}-04 \mathrm{~kg} \mathrm{~mol}=9.42 \mathrm{E}-04 \mathrm{~kg}$

Mass He lost from six monitored MCOs after sampling at $1.25 \mathrm{yr}=6 \mathrm{MCOs} \times \mathrm{DP} \times \mathrm{V} \times 0.936$ $\mathrm{H}_{2} / \mathrm{RT}=6 \mathrm{MCOs} \times 1.0 \mathrm{psia} \times 500 \mathrm{~L} / \mathrm{MCO} \times 0.937 \mathrm{H}_{2} /(14.7 \mathrm{psia} / \mathrm{atm} \times 0.082 \mathrm{~L} \mathrm{~atm} / \mathrm{gmol} \mathrm{K} \mathrm{x}$ $333 \mathrm{~K}) \times 0.001 \mathrm{~kg} / \mathrm{g}=7.00 \mathrm{E}-03 \mathrm{~kg} \mathrm{~mol}=\mathbf{2 . 8 0 \mathrm { E } - \mathbf { 0 2 }} \mathbf{~ k g}$

\section{SAMPLE 5A}

Mass $\mathrm{H}_{2}$ remaining in six monitored MCOs after sampling at $1.25 \mathrm{yr}=1.277 \mathrm{E}-02 \mathrm{~kg} \mathrm{~mol}-$ $4.71 \mathrm{E}-04 \mathrm{~kg} \mathrm{~mol}=1.23 \mathrm{E}-02 \mathrm{~kg} \mathrm{~mol}$

Mass He remaining in six monitored MCOs after sampling at $1.25 \mathrm{yr}$ and before reinerting = $0.1876 \mathrm{~kg} \mathrm{~mol}-7.00 \mathrm{E}-03 \mathrm{~kg}$ mol lost $=1.806 \mathrm{E}-01 \mathrm{~kg} \mathrm{~mol}$

Mass He in reinerted MCOs after sampling and reinerting at $1.25 \mathrm{yr}=1.806 \mathrm{E}-01 \mathrm{~kg} \mathrm{~mol}$ remaining + 7.00E-03 kg mol He lost $+4.71 \mathrm{E}-04 \mathrm{~kg} \mathrm{~mol}$ as $\mathrm{H}_{2}$ lost $=1.881 \mathrm{E}-01 \mathrm{~kg} \mathrm{~mol}$

Concentration of $\mathrm{H}_{2}$ in $\mathrm{MCO}$ at $1.25 \mathrm{yr}$ and after He reinerting $=1.23 \mathrm{E}-02 \mathrm{~kg}$ mol x $100 /(1.23 \mathrm{E}-$ $02 \mathrm{~kg} \mathrm{~mol} \mathrm{H}+1.881 \mathrm{E}-01 \mathrm{~kg} \mathrm{~mol} \mathrm{He}=2.00 \mathrm{E}-01)=6.1 \mathrm{vol} \%$

Concentration of $\mathrm{He}=100-6.1=93.9$ vol $\%$

Pressure inside MCOs at $1.25 \mathrm{yr}$ and after He reinerting $=2.00 \mathrm{E}-01 \mathrm{~kg} \mathrm{~mol} \times 0.0820 \mathrm{~atm} \mathrm{~L} / \mathrm{gmol}$ $\mathrm{K} \times 333 \mathrm{~K} /(500 \mathrm{~L} / \mathrm{MCO} \times 6 \mathrm{MCOs}) \times 1000 \mathrm{~g} / \mathrm{kg}=1.82 \mathrm{~atm}=\mathbf{2 6 . 8} \mathbf{~ p s i a}$

\section{SAMPLE 6B}

Determine mass of $\mathrm{H}_{2}$ in six monitored MCOs at $1.5 \mathrm{yr}$ and no sampling/He reinerting

$0.075=\mathrm{X} \mathrm{H}_{2} /\left(\mathrm{X} \mathrm{H}_{2}+31.0 \mathrm{He}\right)$ based on Duncan and Plys (1998) and Pajunen (2000)

$\mathrm{X}=0.075 \times 31.0 / 0.925=2.513 \mathrm{gmol} / \mathrm{MCO}$ without sampling and reinerting

Amount $\mathrm{H}_{2}$ remaining in six monitored MCOs before sampling at $1.5 \mathrm{yr}$ (sampling and reinerting at $1.25 \mathrm{yr})=2.513 \mathrm{gmol} / \mathrm{MCO} \times 6 \mathrm{MCOs} \times 0.001 \mathrm{~kg} / \mathrm{g}-4.69 \mathrm{E}-04 \mathrm{~kg}$ mol $\times 4$ sampling events $4.71 \mathrm{E}-04 \mathrm{~kg} \mathrm{~mol}$ at $1.25 \mathrm{yr}=1.273 \mathrm{E}-02 \mathrm{~kg} \mathrm{~mol}$

Concentration of $\mathrm{H}_{2}$ at $1.5 \mathrm{yr}$ before sampling $=1.273 \mathrm{E}-02 \mathrm{~kg} \mathrm{~mol} \mathrm{x} 100 /(1.273 \mathrm{E}-02 \mathrm{~kg} \mathrm{~mol} \mathrm{H}$ $+1.881 \mathrm{E}-01 \mathrm{~kg} \mathrm{~mol} \mathrm{He}=2.00 \mathrm{E}-01)=6.3 \mathrm{vol} \%$

Concentration of $\mathrm{He}=100-6.3=93.7$ vol \% 


\section{SNF-5741, Rev. 0}

MCO pressure at $1.5 \mathrm{yr}$ before sampling $=2.00 \mathrm{E}-01 \mathrm{~kg} \mathrm{~mol} \mathrm{x} 0.082 \mathrm{~L}$ atm $/ \mathrm{gmol} \mathrm{K} \mathrm{x} 333 \mathrm{~K} \mathrm{x}$ $1000 \mathrm{~g} / \mathrm{gmol} /(500 \mathrm{~L} / \mathrm{MCO} \times 6 \mathrm{MCOs})=1.82 \mathrm{~atm}=\mathbf{2 6 . 8} \mathbf{p s i a}$

$\mathrm{MCO} /$ sample cart pressure after pressurizing sample cart assuming no temperature effects $=$ $(\mathrm{P} 1 \mathrm{~V} 1+\mathrm{P} 2 \mathrm{~V} 2) /(\mathrm{V} 1+\mathrm{V} 2)=(26.8 \mathrm{psia} \times 500 \mathrm{~L}+14.7 \mathrm{psia} \times 46.4 \mathrm{~L}) /(500 \mathrm{~L}+46.4 \mathrm{~L})=25.8 \mathrm{psia}$

$\mathrm{DP}=26.8 \mathrm{psia}-25.8 \mathrm{psia}=\mathbf{1}$ psia

Mass $\mathrm{H}_{2}$ lost from six monitored MCOs after sampling at $1.5 \mathrm{yr}=6 \mathrm{MCOs} \times \mathrm{DP} \times \mathrm{V} \times 0.063$ $\mathrm{H}_{2} / \mathrm{RT}=6 \mathrm{MCOs} \times 1.0 \mathrm{psia} \times 500 \mathrm{~L} / \mathrm{MCO} \times 0.063 \mathrm{H}_{2} /(14.7 \mathrm{psia} / \mathrm{atm} \times 0.082 \mathrm{~L} \mathrm{~atm} / \mathrm{gmol} \mathrm{K} \times$ $333 \mathrm{~K}) \times 0.001 \mathrm{~kg} / \mathrm{g}=4.71 \mathrm{E}-04 \mathrm{~kg} \mathrm{~mol}=9.42 \mathrm{E}-04 \mathbf{~ k g}$

Mass He lost from six monitored MCOs after sampling at $1.5 \mathrm{yr}=6 \mathrm{MCOs} \times \mathrm{DP} \mathrm{x} \mathrm{Vx}$ $0.937 \mathrm{H}_{2} / \mathrm{RT}=6$ MCOs $\times 1.0 \mathrm{psia} \times 500 \mathrm{~L} / \mathrm{MCO} \times 0.937 \mathrm{H}_{2} /(14.7 \mathrm{psia} / \mathrm{atm} \times 0.082 \mathrm{~L}$ atm $/ \mathrm{gmo}]$ $\mathrm{K} \times 333 \mathrm{~K}) \times 0.001 \mathrm{~kg} / \mathrm{g}=7.00 \mathrm{E}-03 \mathrm{~kg} \mathrm{~mol}=\mathbf{2 . 8 0 E}-\mathbf{0 2} \mathbf{~ k g}$

\section{SAMPLE 6A}

Mass $\mathrm{H}_{2}$ remaining in six monitored MCOs after sampling at $1.5 \mathrm{yr}$ and before reinerting $=$ $1.273 \mathrm{E}-02 \mathrm{~kg} \mathrm{~mol}-4.71 \mathrm{E}-04 \mathrm{~kg} \mathrm{~mol} \mathrm{lost}=1.226 \mathrm{E}-02 \mathrm{~kg} \mathrm{~mol}$

Mass He remaining in six monitored MCOs after sampling at $1.5 \mathrm{yr}$ and before reinerting = $1.881 \mathrm{E}-01 \mathrm{~kg} \mathrm{~mol}-7.00 \mathrm{E}-03 \mathrm{~kg} \mathrm{~mol} \mathrm{lost}=1.811 \mathrm{E}-01 \mathrm{~kg} \mathrm{~mol}$

Mass $\mathrm{He}$ in refill MCOs after sampling and reinerting at $1.5 \mathrm{yr}=1.811 \mathrm{E}-01 \mathrm{He}$ remaining + $7.00 \mathrm{E}-03 \mathrm{~kg} \mathrm{~mol} \mathrm{He}$ lost $+4.71 \mathrm{E}-04 \mathrm{~kg}$ mol lost as $\mathrm{H}_{2}=1.886 \mathrm{E}-01 \mathrm{~kg} \mathrm{~mol}$

Concentration of $\mathrm{H}_{2}$ in $\mathrm{MCO}$ at $1.5 \mathrm{yr}$ and after He reinerting $=1.226 \mathrm{E}-02 \mathrm{~kg} \mathrm{~mol} \mathrm{x}$ $100 /(1.226 \mathrm{E}-02 \mathrm{~kg} \mathrm{~mol} \mathrm{H}+1.886 \mathrm{E}-01 \mathrm{~kg} \mathrm{~mol} \mathrm{He}=2.00 \mathrm{E}-01)=\mathbf{6 . 1}$ vol $\%$

Concentration of $\mathrm{He}=100-6.1=93.9$ vol $\%$

Pressure inside MCOs at $1.5 \mathrm{yr}$ after reinerting $=2.00 \mathrm{E}-01 \mathrm{~kg}$ mol x $0.0820 \mathrm{~L}$ atm $/ \mathrm{gmol} \mathrm{K} \times 333$ $\mathrm{K}) /(500 \mathrm{~L} / \mathrm{MCO} \times 6 \mathrm{MCOs}) \times 1000 \mathrm{~g} / \mathrm{kg}=1.782 \mathrm{~atm}=\mathbf{2 6 . 8} \mathbf{~ p s i a}$

\section{SAMPLE 7B}

Determine mass of $\mathrm{H}_{2}$ in six monitored MCOs at $1.75 \mathrm{yr}$ and no sampling/He reinerting $0.078=\mathrm{X} \mathrm{H}_{2} /\left(\mathrm{X} \mathrm{H}_{2}+31.0 \mathrm{He}\right)$ based on Duncan and Plys (1998) and Pajunen (2000) $\mathrm{X}=0.078 \times 31.0 / 0.922=2.622 \mathrm{gmol} / \mathrm{MCO}$ without sampling and reinerting

Amount $\mathrm{H}_{2}$ remaining in six monitored MCOs before sampling at $1.75 \mathrm{yr}$ (sampling and reinerting at $1.5 \mathrm{yr}$ ) $=2.622$ gmol x 6 MCOs x $0.001 \mathrm{~kg} / \mathrm{g}-4.69 \mathrm{E}-04 \mathrm{~kg}$ mol x 4 sampling events $-4.71 \mathrm{E}-04 \mathrm{~kg} \mathrm{~mol}$ at $1.25 \mathrm{yr}-4.71 \mathrm{E}-04 \mathrm{~kg}$ mol at $1.5 \mathrm{yr}=1.291 \mathrm{E}-02 \mathrm{~kg} \mathrm{~mol}$ 
Concentration of $\mathrm{H}_{2}$ at $1.75 \mathrm{yr}$ and before sampling $=1.291 \mathrm{E}-02 \mathrm{~kg} \mathrm{~mol} \mathrm{x} 100 /(1.291 \mathrm{E}-02 \mathrm{~kg}$ $\mathrm{mol}+1.886 \mathrm{E}-01 \mathrm{~kg} \mathrm{~mol} \mathrm{He}=2.015 \mathrm{E}-01)=6.4 \mathrm{vol} \%$

Concentration of $\mathrm{He}$ at $1.75 \mathrm{yr}$ and before sampling $=100-6.4=93.6 \mathrm{vol} \%$

MCO pressure at $1.75 \mathrm{yr}$ before sampling $=2.015 \mathrm{E}-01 \mathrm{~kg} \mathrm{~mol} \times 0.0820 \mathrm{~L}$ atm $/ \mathrm{gmol} \mathrm{K} \times 333$ $\mathrm{K} /(500 \mathrm{~L} / \mathrm{MCO} \times 6 \mathrm{MCOs} \times 1000 \mathrm{~g} / \mathrm{kg}=1.83 \mathrm{~atm}=27.0 \mathrm{psia}$

$\mathrm{MCO} /$ sample cart pressure after pressurizing sample cart assuming no temperature effects $=$ $(\mathrm{P} 1 \mathrm{~V} 1+\mathrm{P} 2 \mathrm{~V} 2) /(\mathrm{V} 1+\mathrm{V} 2)=(27.0 \mathrm{psia} \times 500 \mathrm{~L}+14.7 \mathrm{psia} \times 46.4 \mathrm{~L}) /(500 \mathrm{~L}+46.4 \mathrm{~L})=26.0 \mathrm{psia}$

$\mathrm{DP}=27.0 \mathrm{psia}-26.0 \mathrm{psia}=1$ psia

Mass $\mathrm{H}_{2}$ lost from six monitored MCOs after sampling at $1.5 \mathrm{yr}=6 \mathrm{MCOs} \times \mathrm{DP} \times \mathrm{V} \times 0.064$ $\mathrm{H}_{2} / \mathrm{RT}=6 \mathrm{MCOs} \times 1.0 \mathrm{psia} \times 500 \mathrm{~L} / \mathrm{MCO} \times 0.064 \mathrm{H}_{2} /(14.7 \mathrm{psia} / \mathrm{atm} \times 0.082 \mathrm{~L} \mathrm{~atm} / \mathrm{gmol} \mathrm{K} \mathrm{x}$ $333 \mathrm{~K}) \times 0.001 \mathrm{~kg} / \mathrm{g}=4.78 \mathrm{E}-04 \mathrm{~kg} \mathrm{~mol}=9.56 \mathrm{E}-04 \mathbf{~ k g}$

Mass He lost from six monitored MCOs after sampling at $1.5 \mathrm{yr}=6 \mathrm{MCOs} \times \mathrm{DP} \times \mathrm{V} \mathrm{x}$ $0.936 \mathrm{H}_{2} / \mathrm{RT}=6 \mathrm{MCOs} \times 1.0 \mathrm{psia} \times 500 \mathrm{~L} / \mathrm{MCO} \times 0.936 \mathrm{H}_{2} /(14.7 \mathrm{psia} / \mathrm{atm} \times 0.082 \mathrm{~L} \mathrm{~atm} / \mathrm{gmol}$ $\mathrm{K} \times 333 \mathrm{~K}) \times 0.001 \mathrm{~kg} / \mathrm{g}=7.00 \mathrm{E}-03 \mathrm{~kg} \mathrm{~mol}=\mathbf{2 . 8 0 E}-02 \mathbf{~ k g}$

\section{SAMPLE 7A}

Mass $\mathrm{H}_{2}$ remaining in six monitored MCOs after sampling at $1.75 \mathrm{yr}$ and before reinerting $=$ $1.291 \mathrm{E}-02 \mathrm{~kg} \mathrm{~mol}-4.78 \mathrm{E}-04 \mathrm{~kg} \mathrm{~mol}$ lost $=1.243 \mathrm{E}-02 \mathrm{~kg} \mathrm{~mol}$

Mass He remaining in six monitored MCOs after sampling at $1.75 \mathrm{yr}$ and before reinerting = $1.886 \mathrm{E}-01 \mathrm{~kg} \mathrm{~mol}-7.00 \mathrm{E}-03 \mathrm{~kg} \mathrm{~mol} \mathrm{lost}=1.816 \mathrm{E}-01 \mathrm{~kg} \mathrm{~mol}$

Mass He in refill MCOs after sampling and reinerting at $1.75 \mathrm{yr}=1.816 \mathrm{E}-01 \mathrm{~kg} \mathrm{~mol} \mathrm{He}$ remaining + 7.00E-03 kg mol He lost $+4.78 \mathrm{E}-04 \mathrm{~kg}$ mol lost as $\mathrm{H}_{2}=1.891 \mathrm{E}-01 \mathrm{~kg} \mathrm{~mol}$

Concentration of $\mathrm{H}_{2}$ in $\mathrm{MCO}$ at $1.75 \mathrm{yr}$ and after He reinerting $=1.243 \mathrm{E}-02 \mathrm{~kg} \mathrm{~mol} \mathrm{x}$ $100 /(1.243 \mathrm{E}-02 \mathrm{~kg} \mathrm{~mol} \mathrm{H}+1.891 \mathrm{E}-01 \mathrm{~kg} \mathrm{~mol} \mathrm{He}=2.015 \mathrm{E}-01)=\mathbf{6 . 2} \mathrm{vol} \%$

Concentration of He at $1.75 \mathrm{yr}$ and after He fill $=100-6.2=93.8 \mathrm{vol} \%$

Pressure inside $\mathrm{MCO}$ at $1.75 \mathrm{yr}$ and after $\mathrm{He}$ refill $=2.015 \mathrm{E}-01 \mathrm{~kg} \mathrm{~mol} \times 0.082 \mathrm{~L}$ atm $/ \mathrm{gmol} \mathrm{K} \mathrm{x}$ $333 \mathrm{~K} \mathrm{x} 1000 \mathrm{~g} / \mathrm{kg} /(500 \mathrm{~L} / \mathrm{MCO} \times 6 \mathrm{MCOs})=1.83 \mathrm{~atm}=\mathbf{2 7 . 0}$ psia

\section{SAMPLE 8B}

Determine mass of $\mathrm{H}_{2}$ in six monitored MCOs at $2.0 \mathrm{yr}$ and no sampling/He reinerting

$0.080=\mathrm{X} \mathrm{H}_{2} /\left(\mathrm{X} \mathrm{H}_{2}+31.0 \mathrm{He}\right)$ based on Duncan and Plys (1998) and Pajunen (2000) 
$\mathrm{X}=0.080 \times 31.0 / 0.920=2.696 \mathrm{gmol} \mathrm{H}_{2} / \mathrm{MCO}$ without sampling and reinerting

Amount $\mathrm{H}_{2}$ remaining in six monitored MCOs before sampling at $2.0 \mathrm{yr}$ (sampling and reinerting at $1.5 \mathrm{yr})=2.696 \mathrm{gmol} \times 6 \mathrm{MCOs} \times 0.001 \mathrm{~kg} / \mathrm{g}-4.69 \mathrm{E}-04 \mathrm{~kg}$ mol x 4 sampling events $-4.71 \mathrm{E}-$ $04 \mathrm{~kg} \mathrm{~mol}$ at $1.25 \mathrm{yr}-4.71 \mathrm{E}-04 \mathrm{~kg} \mathrm{~mol}$ at $1.5 \mathrm{yr}-4.78 \mathrm{E}-04 \mathrm{~kg}$ mol at $1.75 \mathrm{yr}=1.288 \mathrm{E}-02 \mathrm{~kg}$ mol

Concentration of $\mathrm{H}_{2}$ at $2.0 \mathrm{yr}$ and before sampling $=1.288 \mathrm{E}-02 \mathrm{~kg} \mathrm{~mol} \mathrm{x} 100 /(1.288 \mathrm{E}-02 \mathrm{~kg} \mathrm{~mol}$ $+1.891 \mathrm{E}-01 \mathrm{~kg} \mathrm{~mol} \mathrm{He}=2.02 \mathrm{E}-01)=\mathbf{6 . 4}$ vol \%

Concentration of $\mathrm{He}$ at $2.0 \mathrm{yr}$ and before sampling $=100-6.4=93.6 \mathrm{vol} \% \mathrm{He}$

MCO pressure at $2.0 \mathrm{yr}$ before sampling $=2.02 \mathrm{E}-01 \mathrm{~kg} \mathrm{~mol} \times 0.0820 \mathrm{~L} \mathrm{~atm} / \mathrm{gmol} \mathrm{K} \times 333$ $\mathrm{K} /(500 \mathrm{~L} / \mathrm{MCO} \times 6 \mathrm{MCOs} \times 1000 \mathrm{~g} / \mathrm{kg}=1.84 \mathrm{~atm}=27.0 \mathbf{p s i a}$

$\mathrm{MCO} /$ sample cart pressure after pressurizing sample cart assuming no temperature effects = $(\mathrm{P} 1 \mathrm{~V} 1+\mathrm{P} 2 \mathrm{~V} 2) /(\mathrm{V} 1+\mathrm{V} 2)=(27.0 \mathrm{psia} \times 500 \mathrm{~L}+14.7 \mathrm{psia} \times 46.4 \mathrm{~L}) /(500 \mathrm{~L}+46.4 \mathrm{~L})=26.0 \mathrm{psia}$

$\mathrm{DP}=27.0 \mathrm{psia}-26.0 \mathrm{psia}=\mathbf{1}$ psia

Mass $\mathrm{H}_{2}$ lost from six monitored MCOs after sampling at $2.0 \mathrm{yr}=6 \mathrm{MCOs} \times \mathrm{DP} \times \mathrm{V} \mathrm{x} 0.064$ $\mathrm{H}_{2} / \mathrm{RT}=6 \mathrm{MCOs} \times 1.0 \mathrm{psia} \times 500 \mathrm{~L} / \mathrm{MCO} \times 0.063 \mathrm{H}_{2} /(14.7 \mathrm{psia} / \mathrm{atm} \times 0.082 \mathrm{~L} \mathrm{~atm} / \mathrm{gmol} \mathrm{K} \times$ $333 \mathrm{~K}) \times 0.001 \mathrm{~kg} / \mathrm{g}=4.71 \mathrm{E}-04 \mathrm{~kg} \mathrm{~mol}=9.56 \mathrm{E}-04 \mathrm{~kg}$

Mass He lost from six monitored MCOs after sampling at $2.0 \mathrm{yr}=6 \mathrm{MCOs} \times \mathrm{DP} \times \mathrm{V} \times 0.936$ $\mathrm{H}_{2} / \mathrm{RT}=6 \mathrm{MCOs} \times 1.0 \mathrm{psia} \times 500 \mathrm{~L} / \mathrm{MCO} \times 0.936 \mathrm{H}_{2} /(14.7 \mathrm{psia} / \mathrm{atm} \times 0.082 \mathrm{~L} \mathrm{~atm} / \mathrm{gmol} \mathrm{K} \times$ $333 \mathrm{~K}) \times 0.001 \mathrm{~kg} / \mathrm{g}=7.00 \mathrm{E}-03 \mathrm{~kg} \mathrm{~mol}=\mathbf{2 . 8 0 E}-\mathbf{0 2} \mathbf{~ k g}$

\section{SAMPLE 8A}

Mass $\mathrm{H}_{2}$ remaining in six monitored MCOs after sampling at $2.0 \mathrm{yr}$ and before reinerting = $1.288 \mathrm{E}-02 \mathrm{~kg} \mathrm{~mol}-4.71 \mathrm{E}-04 \mathrm{~kg} \mathrm{~mol} \mathrm{lost}=1.283 \mathrm{E}-02 \mathrm{~kg} \mathrm{~mol}=2.57 \mathrm{E}-02 \mathrm{~kg}$

Mass He remaining in six monitored MCOs after sampling at $2.0 \mathrm{yr}$ and before reinerting = $1.891 \mathrm{E}-01 \mathrm{~kg} \mathrm{~mol}-7.00 \mathrm{E}-03 \mathrm{~kg} \mathrm{~mol} \mathrm{lost}=1.821 \mathrm{E}-01 \mathrm{~kg} \mathrm{~mol}$

Mass $\mathrm{He}$ in reinerted MCOs after sampling and reinerting at $2.0 \mathrm{yr}=1.821 \mathrm{E}-01 \mathrm{~kg} \mathrm{~mol} \mathrm{He}$ remaining + 7.00E-03 kg mol He lost $+4.71 \mathrm{E}-04 \mathrm{~kg}$ mol lost as $\mathrm{H}_{2}=1.896 \mathrm{E}-01 \mathrm{~kg}$ mol $=7.58 \mathrm{E}-$ $01 \mathrm{~kg}$

Concentration of $\mathrm{H}_{2}$ in $\mathrm{MCO}$ at $2.0 \mathrm{yr}$ and after $\mathrm{He}$ reinerting $=1.283 \mathrm{E}-02 \mathrm{~kg} \mathrm{~mol} \mathrm{x}$ $100 /\left(1.283 \mathrm{E}-02 \mathrm{~kg} \mathrm{~mol} \mathrm{H}_{2}+1.896 \mathrm{E}-01 \mathrm{~kg} \mathrm{~mol} \mathrm{He}=2.024 \mathrm{E}-01\right)=6.3 \mathrm{vol} \%$

Concentration of He at $2.0 \mathrm{yr}$ and after He reinerting $=100-6.3=93.7 \mathrm{vol} \%$ 
Pressure inside $\mathrm{MCO}$ at $2.0 \mathrm{yr}$ and after He reinerting $=2.024 \mathrm{E}-01 \mathrm{~kg} \mathrm{~mol} \mathrm{x} 0.082 \mathrm{~L}$ atm $/ \mathrm{gmol} \mathrm{K}$ $\times 333 \mathrm{~K} \times 1000 \mathrm{~g} / \mathrm{kg} /(500 \mathrm{~L} / \mathrm{MCO} \times 6 \mathrm{MCOs})=1.84 \mathrm{~atm}=\mathbf{2 7 . 0} \mathbf{p s i a}=1400$ torr

Determine Estimated Hydrated Oxide Radiolysis and Remaining Amount of Free Water in Monitored MCOs at 2 Years

Based on adjusted approximations from sections 5.5.1 and 5.5.2 of Duncan and Plys (1998), assume $0.005 \%$ of $\mathrm{Al}(\mathrm{OH})_{3}$ and $6.0 \%$ of $\mathrm{UO}_{2}(\mathrm{OH})_{2}$ are decomposed by radiation to $\mathrm{Al}_{2} \mathrm{O}_{3}$, $\mathrm{UO}_{3}$, and hydrogen during the two years of $\mathrm{MCO}$ monitoring (private communication with $\mathrm{A}$. $\mathrm{L}$. Pajunen of Fluor Hanford). This approximation gives a larger free water inside the MCO since a small amount of hydrogen is produced by radiolysis of the bound (hydrated oxide) water. The oxygen is gettered by reaction with uranium metal to form additional $\mathrm{UO}_{2}$.

Determine amount of $\mathrm{Al}_{2} \mathrm{O}_{3}, \mathrm{UO}_{2}$, and hydrogen produced by radiolytic decomposition of $\mathrm{Al}(\mathrm{OH})_{3}$ in six monitored MCOs (hydrogen included in above estimates as part of overall hydrogen generation from modeling in Duncan and Plys (1998)):

$2 \mathrm{Al}(\mathrm{OH})_{3} \rightarrow \mathrm{Al}_{2} \mathrm{O}_{3}+3 \mathrm{H}_{2} \mathrm{O} \rightarrow \mathrm{Al}_{2} \mathrm{O}_{3}+3 \mathrm{H}_{2}+1.5 \mathrm{O}_{2}$

$1.5 \mathrm{O}_{2}+1.5 \mathrm{U} \rightarrow 1.5 \mathrm{UO}_{2}$ (gettering)

$4.15 \mathrm{~kg} \mathrm{Al}(\mathrm{OH})_{3} / 78 \mathrm{~g} / \mathrm{gmol} \rightarrow 0.995 \times 4.15 \mathrm{~kg} \mathrm{Al}(\mathrm{OH})_{3}+0.005 \times 4.15 \mathrm{~kg} \mathrm{Al}(\mathrm{OH})_{3} /(78 \mathrm{~g} / \mathrm{gmol})$ x $1 \mathrm{gmol} \mathrm{Al} \mathrm{O}_{3} / 2 \mathrm{gmol} \mathrm{Al}(\mathrm{OH})_{3} \times 102 \mathrm{~g} \mathrm{Al}_{2} \mathrm{O}_{3} / \mathrm{gmol}\left(\mathrm{kg} \mathrm{Al}_{2} \mathrm{O}_{3}\right)+0.005 \times 4.15 \mathrm{~kg} \mathrm{Al}(\mathrm{OH})_{3} /(78$ $\mathrm{g} / \mathrm{gmol}) \times 3 \mathrm{gmol} \mathrm{H}_{2} / 2 \mathrm{gmol} \times 2 \mathrm{~g} \mathrm{H}_{2} / \mathrm{gmol}\left(\mathrm{kg} \mathrm{H}_{2}\right)+1.5 \mathrm{gmol} \mathrm{O}{ }_{2}$ or $\mathrm{UO}_{2} / 2 \mathrm{gmol} \mathrm{Al}(\mathrm{OH})_{3} \mathrm{x}$ $0.005 \times 4.15 \mathrm{~kg} \mathrm{Al}(\mathrm{OH})_{3} /(78 \mathrm{~g} / \mathrm{gmol})$

Remaining $\mathrm{Al}(\mathrm{OH})_{3}=0.995 \times 4.15 \mathrm{~kg}=4.13 \mathrm{~kg}$ or $6.88 \mathrm{E}-01 \mathrm{~kg} / \mathrm{MCO}$

Amount $\mathrm{Al}_{2} \mathrm{O}_{3}$ produced $=0.005 \times 4.15 \mathrm{~kg} \mathrm{Al}(\mathrm{OH})_{3} /(78 \mathrm{~g} / \mathrm{gmol}) \times 1 \mathrm{gmol} \mathrm{Al}_{2} \mathrm{O}_{3} / 2 \mathrm{gmol}$ $\mathrm{Al}(\mathrm{OH})_{3} \times 102 \mathrm{~g} \mathrm{Al}_{2} \mathrm{O}_{3} / \mathrm{gmol}=1.35 \mathrm{E}-02 \mathrm{~kg}$ or $2.26 \mathrm{E}-03 \mathrm{~kg} / \mathrm{MCO}$

Amount hydrogen produced $=0.005 \times 4.15 \mathrm{~kg} \mathrm{Al}(\mathrm{OH})_{3} /(78 \mathrm{~g} / \mathrm{gmol}) \times 3 \mathrm{gmol} \mathrm{H}_{2} / 2 \mathrm{gmol}$ $\mathrm{Al}(\mathrm{OH})_{3} \times 2 \mathrm{~g} \mathrm{H}_{2} / \mathrm{gmol}=7.98 \mathrm{E}-04 \mathbf{~ k g ~ H}_{2}$ or $1.33 \mathrm{E}-04 \mathrm{~kg} \mathrm{H}_{2} / \mathrm{MCO}$

Amount of new $\mathrm{UO}_{2}$ produced $=0.005 \times 4.15 \mathrm{~kg} \mathrm{Al}(\mathrm{OH})_{3} /(78 \mathrm{~g} / \mathrm{gmol}) \times 1.5 \mathrm{gmol} \mathrm{UO} / 2 \mathrm{gmol}$ $\mathrm{Al}(\mathrm{OH})_{3}$ x $270 \mathrm{~g} \mathrm{UO}_{2} / \mathrm{gmol}=\mathbf{5 . 3 9 E - 0 2 ~} \mathbf{~ k g ~ U \mathbf { O } _ { 2 }}$ or $8.98 \mathrm{E}-03 \mathrm{~kg} / \mathrm{MCO}$

Determine amount of $\mathrm{UO}_{3}, \mathrm{UO}_{2}$, and hydrogen produced at two years by radiolytic decomposition of $\mathrm{UO}_{2}(\mathrm{OH})_{2}$ in six monitored MCOs (hydrogen included in above estimates as part of overall hydrogen generation from modeling in Duncan and Plys (1998)):

$\mathrm{UO}_{2}(\mathrm{OH})_{2} \rightarrow \mathrm{UO}_{3}+\mathrm{H}_{2} \mathrm{O} \rightarrow \mathrm{UO}_{3}+\mathrm{H}_{2}+0.5 \mathrm{O}_{2}$

$\mathrm{O}_{2}+\mathrm{U} \rightarrow \mathrm{UO}_{2}$ 
$14.5 \mathrm{~kg} \mathrm{UO}_{2}(\mathrm{OH})_{2} / 304 \mathrm{~g} / \mathrm{gmol} \rightarrow 0.94 \times 14.5 \mathrm{~kg} \mathrm{UO}_{2}(\mathrm{OH})_{2}+0.06 \times 14.5 \mathrm{~kg} \mathrm{UO}_{2}(\mathrm{OH})_{2} / 304$ $\mathrm{g} / \mathrm{gmol} \times 1 \mathrm{gmol} \mathrm{UO} / \mathrm{gmol} \mathrm{UO}_{2}(\mathrm{OH})_{2} \times 286 \mathrm{~g} \mathrm{UO}_{3} / \mathrm{gmol}\left(\mathrm{kg} \mathrm{UO}_{3}\right)+0.06 \times 14.5 \mathrm{~kg}$ $\mathrm{UO}_{2}(\mathrm{OH})_{2} / 304 \mathrm{~g} / \mathrm{gmol} \mathrm{x} 1 \mathrm{gmol} \mathrm{H} / \mathrm{gmol} \mathrm{UO}_{2}(\mathrm{OH})_{2} \times 2 \mathrm{~g} \mathrm{H}_{2} / \mathrm{gmol}\left(\mathrm{kg} \mathrm{H}_{2}\right)+0.06 \times 14.5 \mathrm{~kg}$ $\mathrm{UO}_{2}(\mathrm{OH})_{2} / 304 \mathrm{~g} / \mathrm{gmol} \times 0.5 \mathrm{gmol} \mathrm{O} / \mathrm{gmol} \mathrm{UO}_{2}(\mathrm{OH})_{2} \times 1 \mathrm{gmol} \mathrm{UO} / \mathrm{gmol} \mathrm{O}_{2} \times 270 \mathrm{~g} \mathrm{UO} / \mathrm{gmol}$

Remaining $\mathrm{UO}_{2}(\mathrm{OH})_{2}=0.94 \times 14.5 \mathrm{~kg}=1.36 \mathrm{E}+01 \mathrm{~kg}$ or $2.27 \mathrm{E}+00 \mathrm{~kg} / \mathrm{MCO}$

Amount $\mathrm{UO}_{3}$ produced $=0.06 \times 14.5 \mathrm{~kg} \mathrm{UO} 2(\mathrm{OH})_{2} / 304 \mathrm{~g} / \mathrm{gmol} \times 1 \mathrm{gmol} \mathrm{UO} / \mathrm{gmol} \mathrm{UO}_{2}(\mathrm{OH})_{2} \times$ $286 \mathrm{~g} / \mathrm{gmol} \mathrm{UO}_{3}=\mathbf{8 . 1 8 E - 0 1 ~} \mathbf{~ g g}$ or $1.36 \mathrm{E}-01 \mathrm{~kg} / \mathrm{MCO}$

Amount of $\mathrm{UO}_{2}$ produced $=0.06 \times 14.5 \mathrm{~kg} \mathrm{UO}_{2}(\mathrm{OH})_{2} / 304 \mathrm{~g} / \mathrm{gmol} \times 0.5 \mathrm{gmol} \mathrm{O} / \mathrm{gmol}$ $\mathrm{UO}_{2}(\mathrm{OH})_{2} \times 1 \mathrm{gmol} \mathrm{UO}_{2} / \mathrm{gmol} \mathrm{O}_{2} \times 270 \mathrm{~g} \mathrm{UO}_{2} / \mathrm{gmol}=3.86 \mathrm{E}-01 \mathrm{~kg}$ or $6.44 \mathrm{E}-02 \mathrm{~kg} / \mathrm{MCO}$

Total $\mathrm{UO}_{2}$ in 6 monitored $\mathrm{MCOs}=1.90 \mathrm{E}+00 \mathrm{~kg}(\mathrm{C}-7)+5.4 \mathrm{E}-02 \mathrm{~kg}+3.86 \mathrm{E}-01 \mathrm{~kg}=2.34 \mathrm{E}+00$ $\mathrm{kg}$ or $3.90 \mathrm{E}-01 \mathrm{~kg} / \mathrm{MCO}$

Amount hydrogen produced $=0.06 \times 14.5 \mathrm{~kg} \mathrm{UO}_{2}(\mathrm{OH})_{2} / 304 \mathrm{~g} / \mathrm{gmol} \times 1 \mathrm{gmol} \mathrm{H} / 2 / \mathrm{gmol}$ $\mathrm{UO}_{2}(\mathrm{OH})_{2} \times 2 \mathrm{~g} \mathrm{H}_{2} / \mathrm{gmol}=\mathbf{5 . 7 2 E - 0 3 ~} \mathbf{~ k g}$

Total hydrogen produced from radiolysis of $\mathrm{Al}(\mathrm{OH})_{3}$ and $\mathrm{UO}_{2}(\mathrm{OH})_{2}$ in six monitored $\mathrm{MCOs}=$

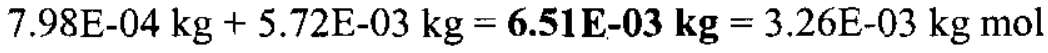

Total hydrogen produced/monitored $\mathrm{MCO}$ from radiolysis of $\mathrm{Al}(\mathrm{OH})_{3}$ and $\mathrm{UO}_{2}(\mathrm{OH})_{2}=6.51 \mathrm{E}$ $03 \mathrm{~kg} / 6 \mathrm{MCOs}=1.09 \mathrm{E}-03 \mathrm{~kg}=\mathbf{5 . 4 3 E - 0 4 ~} \mathbf{~ g g ~ m o l}$

Determine hydrated oxide water resulting from decomposition of $\mathrm{Al}(\mathrm{OH})_{3}$ and $\mathrm{UO}_{2}(\mathrm{OH})_{2}$

Free water/MCO at time zero based on $6.0 \mathrm{vol} \% \mathrm{H}_{2}=1.64 \mathrm{E}+02 \mathrm{~g}=9.11 \mathrm{E}+00$ gmol (see Stream IC 003)

Amount of $\mathrm{H}_{2} / \mathrm{MCO}$ at time zero $=\mathbf{1 . 9 7 8 E}+\mathbf{0 0} \mathrm{gmol} \mathrm{H}_{2} / \mathrm{MCO}($ See Stream IC-003)

Amount of $\mathrm{H}_{2} / \mathrm{MCO}$ at two $\mathrm{yr}$ w/o sampling (assume the same amount $\mathrm{H}_{2}$ produced with sampling $)=\mathbf{2 . 6 9 6 E}+00 \mathrm{gmol} \mathrm{H}_{2} / \mathrm{MCO}$ (See 8B)

Change of $\mathrm{H}_{2}$ from time zero to two yr with correction for decomposition of hydrated oxides = $2.696 \mathrm{E}+00-1.978 \mathrm{E}+00-5.43 \mathrm{E}-01 \mathrm{gmol}=1.75 \mathrm{E}-01 \mathbf{g m o l ~ H}_{2} / \mathrm{MCO}$

Determine amount of free water equivalent to change of $\mathrm{H}_{2}$ value

$\mathrm{H}_{2} \mathrm{O} \rightarrow \mathrm{H}_{2}+0.5 \mathrm{O}_{2}$

1.75E-01 gmol $\mathrm{H}_{2} \times 1$ gmol $\mathrm{H}_{2} 0 / \mathrm{gmol} \mathrm{H}_{2} \times 18 \mathrm{~g} \mathrm{H}_{2} \mathrm{O} / \mathrm{gmol}=\mathbf{3 . 1 5 E}+\mathbf{0 0} \mathrm{g} \mathrm{H}_{2} \mathrm{O} / \mathrm{MCO}$

Water remaining/monitored $\mathrm{MCO}$ at $2 \mathrm{yr}=1.64 \mathrm{E}+02-\mathbf{3 . 1 5 E}+00=1.61 \mathrm{E}+02 \mathrm{~g}$ 
SNF-5741, Rev. 0

Water remaining in six monitored $\mathrm{MCOs}=1.61 \mathrm{E}+02 \mathrm{~g} / \mathrm{MCO} \times 6 \mathrm{MCOs}=9.66 \mathrm{E}+02 \mathrm{~g}=\mathbf{9 . 6 6 E - 0 1 ~} \mathbf{k g}$

\subsubsection{C-13 Monitored MCOs to Welding}

Assume same composition and number of MCOs as Stream C-12. See Stream C-52 for composition after venting, 100 psi pressure unit replacement, and helium reinerting.

\subsubsection{C-14 MCOs to Welding}

Sum of Stream C-6 for the 392 MCOs going directly to welding and Stream C-52 for the six monitored MCOs at $2 \mathrm{yr}$.

\subsubsection{C-15 MCOs to Canister Cover Cap Welding}

Optional leak test results in no change of MCO composition. MCO composition same as Stream C-14.

\subsubsection{C-16 Argon Welding Gas Supply}

Argon is supplied from cylinders as the cover gas for welding of the MCO cover caps and cover plate welds. Assume the following conditions:

- four weld passes per MCO for the cover cap weld and two weld passes per MCO for the cover plate weld(private communication with T. A. Delucchi of COGEMA)

- an argon cover gas usage rate of 40 standard $\mathrm{ft}^{3} / \mathrm{hr}$ for each cover cap weld and 20 standard $\mathrm{ft}^{3} / \mathrm{hr}$ for each cover plate weld (private communication with T. A. Delucchi of COGEMA)

- a $0.5 \mathrm{hr} /$ pass welding rate for both the cover cap and cover plate (private communication with T. A. Delucchi of COGEMA)

- $1 \frac{1}{2} \%$ of MCOs $(0.015 \times 398=6$ MCOs $)$ experience a cover cap weld test failure and no MCOs experience a cover plate weld test failure based on dye penetrant test (private communication with T. A. Delucchi of COGEMA)

- failed cover cap rewelding is $2.5 \mathrm{~cm}$ (1 in)/weld failed MCO (private communication with T. A. Delucchi of COGEMA)

Volume of argon gas $=\left(40 \mathrm{ft}^{3} / \mathrm{hr} \times 0.5 \mathrm{hr} /\right.$ pass $\times 4$ passes $/ \mathrm{MCO}$ cover cap $+20 \mathrm{ft}^{3} / \mathrm{h} \times 0.5$ $\mathrm{hr} /$ pass $\times 2$ passes/MCO cover plate) $\times 398 \mathrm{MCOs}=3.98 \mathrm{E}+04 \mathrm{std}^{3}=\mathbf{1 . 1 3 E}+\mathbf{0 3} \mathbf{s t d ~}^{3}$

Mass of argon gas $=3.98 \mathrm{E}+04 \mathrm{ft}^{3} \times 28.3 \mathrm{~L} / \mathrm{ft}^{3} \times 273 \mathrm{~K} / 288 \mathrm{~K} \mathrm{x} \mathrm{gmol} / 22.4 \mathrm{~L} \times 39.94 \mathrm{~g} / \mathrm{gmol}=$ $1.90 \mathrm{E}+03 \mathrm{~kg}$

\subsubsection{C-17 Deleted}

\subsubsection{C-18 Deleted}


SNF-5741, Rev. 0

\subsubsection{C-19 MCO Welding/Grinding Waste}

Assume the following:

- mass of welding waste (beads and wire fragments) is $0.2 \mathrm{~g} /$ weld (private communication with T. A. Delucchi of COGEMA)

- one spool of weld wire [ 4 in dia $x 2.0$ in high, $2.0 \mathrm{lb}$ initial total wt, and $0.35 \mathrm{lb}$ final weight (2.0-1.65 lb $=160 \mathrm{~g}$ weld wire)] is used per MCO cover cap weld (private communication with T. A. Delucchi of COGEMA)

- 4.5 weld rods (1/16 in dia $\times 36$ in long) are used for each cover plate weld; 6 in lengths of weld $\operatorname{rod}(2.33 \mathrm{~g} / \mathrm{rod}$ ) result as waste (private communication with T. A. Delucchi of COGEMA)

- $1 \frac{1}{2} \%$ of MCOs ( 6 total) have a failed weld section ( 1 in long) based on dye penetrant test and require grinding and rewelding; grinding and rewelding waste $=4.0 \mathrm{~g}$ per failed $\mathrm{MCO}$ weld (private communication with T. A. Delucchi of COGEMA)

- Welding beads, weld wire, weld rod, and weld grinding waste is steel with a density of 7.89 $\mathrm{g} / \mathrm{cc}$. Spool is fabricated from plastic (private communication with T. A. Delucchi of COGEMA)

- The Port \# 2 cover plate (5.5 in dia x 1.0 in) and 4 bolts (2.9 in long x 0.49 in dia) of the MCO shield plug are removed before welding of the cover cap in order to allow future access to the sample valve (Smith 1999, H-2-828048, Rev. 3, and private conversation with L. H. Goldmann of Fluor Hanford). Assume cover plate and bolts discarded to waste.

- The 100 psi pressure sending unit ( 3 in dia $\times 2$ in high and $2.5 \mathrm{lb}$ ) and readout unit $(2.75$ in dia $\mathrm{x} 1$ in high and $0.5 \mathrm{lb}$ ) in Port \#1 of shield plug of monitored MCOs is replaced with a 600 psi sending unit before welding cover cap. The 100 psi pressure units are discarded to solid waste. Pressure unit size is based on private communication with D. G. Douglas of Vista Research Inc.

Mass of welding waste $=0.2 \mathrm{~g} /$ weld $\times 6$ welds $/ \mathrm{MCO} \times 398 \mathrm{MCOs}=\mathbf{4 7 8} \mathbf{g}$ Mass of weld wire/rod waste $=(160 \mathrm{~g}$ unused wire $+10 \mathrm{~g}$ unused weld $\mathrm{rod} / \mathrm{MCO}) \times 398 \mathrm{MCOs}$

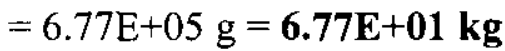

Mass weld spool waste $=60 \mathrm{~g}$ plastic spool $/ \mathrm{MCO} \times 398 \mathrm{MCOs}=\mathbf{2 . 3 9 E}+\mathbf{0 1} \mathbf{~ k g}$

Volume of weld spool waste $=(4$ in dia $\times 2$ in high $=412 \mathrm{cc} / \mathrm{MCO}) \times 398 \mathrm{MCOs}=1.64 \mathrm{E}+05 \mathrm{cc}$ $=1.64 \mathrm{E}-01 \mathrm{~m}^{3}$.

Volume unused weld rod $=1 / 16$ in dia $\times 6.0$ in long $\times 4.5 \mathrm{rods} / \mathrm{MCO}$ x $398 \mathrm{MCOs}=$ 33 in $^{3}=5.43 \mathrm{E}-04 \mathrm{~m}^{3}$

Volume of cover plate $=5.5$ in dia $x 1$ in $x 398 \mathrm{MCOs}=9.45+03 \mathrm{in}^{3}=\mathbf{1 . 5 5 E}-\mathbf{0 1} \mathbf{m}^{3}$

Volume of bolts $=0.49$ in dia $\times 2.9$ in long x 4 bolts $/ \mathrm{MCO} \times 398 \mathrm{MCOs}=8.70 \mathrm{E}+02 \mathrm{in}^{3}=$

\subsection{E-02 $\mathrm{m}^{3}$}

Volume of 100 psi pressure sending units $=3$ in dia $\times 2$ in high/MCO $\times 6$ MCOs $=85$ in $^{3}=$ $1.39 \mathrm{E}-03 \mathrm{~m}^{3}$

Grinding and reweld waste mass $=4.0 \mathrm{~g} /$ failed $\mathrm{MCO}$ weld $\mathrm{x} 6$ failed $\mathrm{MCO}$ welds $=\mathbf{2 4} \mathbf{g}$

Mass of cover plate $=7.0 \mathrm{lb} / \mathrm{MCO} \times 398 \mathrm{MCOs} \times 454 \mathrm{~g} / \mathrm{lb} \times 0.001 \mathrm{~kg} / \mathrm{g}=\mathbf{1 . 2 6 E}+\mathbf{0 3} \mathbf{~ k g}$ Mass of cover plate bolts $=1.29 \mathrm{E}-01 \mathrm{lb} /$ bolt $\times 4$ bolts $/$ MCO x 398 MCOs x $454 \mathrm{~g} / \mathrm{lb} \times 0.001 \mathrm{~kg} / \mathrm{g}$ $=9.32 \mathrm{E}+01 \mathrm{~kg}$ 
Volume of 100 psi pressure readout units $=2.75$ in dia $\times 1$ in high $\times 6 \mathrm{MCOs}=36 \mathrm{in}^{3}=5.84 \mathrm{E}-$ $04 \mathrm{~m}^{3}$

Mass of $100 \mathrm{psi}$ pressure sending units $=2.5 \mathrm{lb} / \mathrm{MCO} \times 6 \mathrm{MCOs} \times 4545 / \mathrm{lb} \times .001 \mathrm{~kg} / \mathrm{g}=6.81 \mathrm{~kg}$ Mass of $100 \mathrm{psi}$ readout units $=0.5 \mathrm{lb} / \mathrm{MCO} \times 6 \mathrm{MCOs}=3.0 \mathrm{lb}=1.4 \mathrm{~kg}$

Welding waste mass steel $=0.48 \mathrm{~kg}+67.7 \mathrm{~kg}+0.024 \mathrm{~kg}=6.82 \mathrm{E}+01 \mathrm{~kg}$ Mass MCO cover plate $/$ bolts $=1.26 \mathrm{E}+03 \mathrm{~kg}+9.32 \mathrm{E}+01 \mathrm{~kg}=1.35 \mathrm{E}+\mathbf{0 3} \mathbf{~ k g}$ Mass plastic weld spool $=\mathbf{2 . 3 9 E}+01 \mathbf{~ k g}$ Waste mass total $=68.2 \mathrm{~kg}+1.35 \mathrm{E}+03+23.9 \mathrm{~kg}+6.8 \mathrm{~kg}+1.4 \mathrm{~kg}=\mathbf{1 . 4 5 E}+\mathbf{0 3} \mathbf{~ k g}$ Waste volume $=1.64 \mathrm{E}-01 \mathrm{~m}^{3}+5.43 \mathrm{E}-04 \mathrm{~m}^{3}+1.55 \mathrm{E}-01 \mathrm{~m}^{3}+1.43 \mathrm{E}-02 \mathrm{~m}^{3}+1.39 \mathrm{E}-03 \mathrm{~m}^{3}+$ $5.84 \mathrm{E}-04 \mathrm{~m}^{3}=3.35 \mathrm{E}-01 \mathrm{~m}^{3}$ Average density $=1.45 \mathrm{E}+03 \mathrm{~kg} / 3.35 \mathrm{E}-01 \mathrm{~m}^{3}=4.33 \mathrm{E}+03 \mathrm{~kg} / \mathrm{m}^{3}=4.33 \mathrm{~g} / \mathrm{cc}$

\subsubsection{C-20 MCO Weld Preparation and Examination Waste}

Assume $2 \mathrm{~kg} / \mathrm{MCO}$ of solid waste from weld preparation and examination. Assume there will be no liquid. Solid waste density is assumed as $0.6 \mathrm{~g} / \mathrm{cc}$ before and after use.

Total solid mass $=2 \mathrm{~kg} / \mathrm{MCO} \times 398 \mathrm{MCOs}=796 \mathbf{~ k g}$ Total solid volume $=796 \mathrm{~kg} / 0.6 \mathrm{E} 03 \mathrm{~kg} / \mathrm{m}^{3}=1.33 \mathrm{~m}^{3}$

\subsubsection{C-21 Welded MCO to Interim Storage}

There is no change within the MCOs during welding. Before removal of the weld sealed MCO from the weld station, a magnetic pressure readout unit is installed in the cover cap of all 398 MCOs. The sludge content of the 392 MCOs from direct welding and six monitored MCOs has been adjusted to two yr of interim storage. This adjustment is based on radiolysis of the hydrated oxide (See Stream C-12 for description of radiolysis) and helium reinerting. The below calculations are for the 392 MCOs going directly to welding. Total for Stream C-21 is sum of Stream C-52 for six monitored MCOs and below calculations for 392 direct welded MCOs.

Remaining $\mathrm{Al}(\mathrm{OH})_{3}=0.995 \times 271 \mathrm{~kg}=\mathbf{2 6 9 . 5} \mathbf{~ k g}$ or $6.88 \mathrm{E}-01 \mathrm{~kg} / \mathrm{MCO}$

Amount $\mathrm{Al}_{2} \mathrm{O}_{3}$ produced $=0.005 \times 271 \mathrm{~kg} \mathrm{Al}(\mathrm{OH})_{3} /(78 \mathrm{~g} / \mathrm{gmol}) \times 1$ gmol $\mathrm{Al}_{2} \mathrm{O}_{3} / 2 \mathrm{gmol} \mathrm{Al}(\mathrm{OH})_{3}$ $\mathrm{x} 102 \mathrm{~g} \mathrm{Al}_{2} \mathrm{O}_{3} / \mathrm{gmol}=\mathbf{8 . 8 6 E}-01 \mathrm{~kg}$ or $2.26 \mathrm{E}-03 \mathrm{~kg} / \mathrm{MCO}$

Amount hydrogen produced $=0.005 \times 271 \mathrm{~kg} \mathrm{Al}(\mathrm{OH})_{3} /(78 \mathrm{~g} / \mathrm{gmol}) \times 3 \mathrm{gmol} \mathrm{H} \mathrm{H}_{2} / 2 \mathrm{gmol} \mathrm{Al}(\mathrm{OH})_{3}$ x $2 \mathrm{~g} \mathrm{H}_{2} / \mathrm{gmol}=\mathbf{5 . 2 1 \mathrm { E } - 0 2} \mathbf{~ k g ~ H}$ or $1.33 \mathrm{E}-04 \mathrm{~kg} \mathrm{H}_{2} / \mathrm{MCO}$

Amount of new $\mathrm{UO}_{2}$ produced $=.005 \times 271 \mathrm{~kg} \mathrm{Al}(\mathrm{OH})_{3} /(78 \mathrm{~g} / \mathrm{gmol}) \times 1.5 \mathrm{gmol} \mathrm{UO}_{2} / 2 \mathrm{gmol}$ $\mathrm{Al}(\mathrm{OH})_{3} \times 270 \mathrm{~g} \mathrm{UO}_{2} / \mathrm{gmol}=\mathbf{3 . 5 2 E}+00 \mathbf{~ k g ~ U O} \mathbf{O}_{2}$ or $8.98 \mathrm{E}-03 \mathrm{~kg} / \mathrm{MCO}$

Remaining $\mathrm{UO}_{2}(\mathrm{OH})_{2}=0.94 \times 948 \mathrm{~kg}=\mathbf{8 . 9 1 E}+\mathbf{0 2} \mathbf{~ k g}$ or $2.27 \mathrm{E}+00 \mathrm{~kg} / \mathrm{MCO}$

Amount $\mathrm{UO}_{3}$ produced $=0.06 \times 948 \mathrm{~kg} \mathrm{UO}_{2}(\mathrm{OH})_{2} / 304 \mathrm{~g} / \mathrm{gmol} \times 1 \mathrm{gmol} \mathrm{UO} / / \mathrm{gmol} \mathrm{UO}_{2}(\mathrm{OH})_{2} \mathrm{x}$ $286 \mathrm{~g} / \mathrm{gmol} \mathrm{UO}_{3}=\mathbf{5 . 3 1 E}+01 \mathrm{~kg}$ or $1.36 \mathrm{E}-01 \mathrm{~kg} / \mathrm{MCO}$ 
SNF-5741, Rev. 0

$\begin{array}{llll}\mathrm{Fe}(\mathrm{OH})_{3} & = & 2.75 \mathrm{E}+01 \mathrm{~kg} & \text { same as Stream C-6 } \\ \mathrm{UH}_{3} & = & 3.72 \mathrm{E}+02 \mathrm{~kg} & \text { same as Stream C-6 } \\ \mathrm{UO}_{4} \cdot 4 \mathrm{H}_{2} \mathrm{O} & = & 0.00 \mathrm{E}+00 \mathrm{~kg} & \text { same as Stream C-6 } \\ \mathrm{UO}_{4} \cdot 2 \mathrm{H}_{2} \mathrm{O} & = & 9.45 \mathrm{E}+02 \mathrm{~kg} & \text { same as Stream C-6 }\end{array}$

Amount of new $\mathrm{UO}_{2}$ produced $=0.06 \times 948 \mathrm{~kg} \mathrm{UO}_{2}(\mathrm{OH})_{2} / 304 \mathrm{~g} / \mathrm{gmol} \times 0.5 \mathrm{gmol} \mathrm{O}_{2} / \mathrm{gmol}$ $\mathrm{UO}_{2}(\mathrm{OH})_{2} \times 1 \mathrm{gmol} \mathrm{UO} / \mathrm{gmol} \mathrm{O}_{2} \times 270 \mathrm{~g} \mathrm{UO}_{2} / \mathrm{gmol}=\mathbf{2 . 5 2 E}+01 \mathrm{~kg}$ or $6.44 \mathrm{E}-02 \mathrm{~kg} / \mathrm{MCO}$

Total $\mathrm{UO}_{2}$ in $392 \mathrm{MCOs}=1.24 \mathrm{E}+02 \mathrm{~kg}(\mathrm{C}-6)+3.5 \mathrm{E}+00 \mathrm{~kg}+2.52 \mathrm{E}+01 \mathrm{~kg}=\mathbf{1 . 5 3 E}+\mathbf{0 2} \mathbf{k g}$ or $3.90 \mathrm{E}+01 \mathrm{~kg} / \mathrm{MCO}$

Amount hydrogen produced $=0.06 \times 948 \mathrm{~kg} \mathrm{UO}_{2}(\mathrm{OH})_{2} / 304 \mathrm{~g} / \mathrm{gmol} \times 1 \mathrm{gmol} \mathrm{H}_{2} / \mathrm{gmol}$ $\mathrm{UO}_{2}(\mathrm{OH})_{2} \times 2 \mathrm{~g} \mathrm{H}_{2} / \mathrm{gmol}=\mathbf{3 . 7 4 E - 0 1 ~} \mathbf{~ k g}$

Total hydrogen produced from radiolysis of $\mathrm{Al}(\mathrm{OH})_{3}$ and $\mathrm{UO}_{2}(\mathrm{OH})_{2}$ in 392 direct welded MCOs $=5.21 \mathrm{E}-02 \mathrm{~kg}+3.74 \mathrm{E}-01 \mathrm{~kg}=4.26 \mathrm{E}-01 \mathrm{~kg}=2.13 \mathrm{E}-01 \mathrm{~kg} \mathrm{~mol}$

Total hydrogen produced/direct welded $\mathrm{MCO}$ from radiolysis of $\mathrm{Al}(\mathrm{OH})_{3}$ and $\mathrm{UO}_{2}(\mathrm{OH})_{2}=$ $4.26 \mathrm{E}-01 \mathrm{~kg} / 392 \mathrm{MCOs}=1.09 \mathrm{E}-03 \mathrm{~kg}=\mathbf{5 . 4 3 E}-\mathbf{0 4} \mathbf{~ k g ~ m o l}$

Determine hydrated oxide water resulting from decomposition of $\mathrm{Al}(\mathrm{OH})_{3}$ and $\mathrm{UO}_{2}(\mathrm{OH})_{2}$

Free water/MCO at time zero based on $6.0 \mathrm{vol} \% \mathrm{H}_{2}=1.64 \mathrm{E}+02 \mathrm{~g}=9.11 \mathrm{E}+00 \mathrm{gmol}$ (see Stream IC-003)

Amount of $\mathrm{H}_{2} / \mathrm{MCO}$ at time zero $=\mathbf{1 . 9 7 8 E}+\mathbf{0 0} \mathrm{gmol}_{2} / \mathrm{MCO}$ (See Stream IC-003)

Amount of $\mathrm{H}_{2} / \mathrm{MCO}$ at two $\mathrm{yr}=0.08 \times 31.0 / 0.92=2.696 \mathrm{E}+00 \mathrm{gmol} \mathrm{H}_{2} / \mathrm{MCO}$ based on Duncan and Plys (1998) and Pajunen (2000)

Change of $\mathrm{H}_{2}$ from time zero to two years with correction for decomposition of hydrated oxides $=2.696 \mathrm{E}+00 \mathrm{gmol}-1.978 \mathrm{E}+00 \mathrm{gmol}-5.43 \mathrm{E}-01 \mathrm{gmol}=1.75 \mathrm{E}-01 \mathbf{g m o l ~} \mathbf{H}_{2} / \mathrm{MCO}$

Determine amount of free water equivalent to change of $\mathrm{H}_{2}$ value

1.75E-01 gmol $\mathrm{H}_{2} \times 1$ gmol $\mathrm{H}_{2} \mathrm{O} / \mathrm{gmol} \mathrm{H}_{2} \times 18 \mathrm{~g} \mathrm{H}_{2} \mathrm{O} / \mathrm{gmol}=\mathbf{3 . 1 5 E}+00 \mathrm{~g} \mathrm{H}_{2} \mathrm{O} / \mathrm{MCO}$

Water remaining/direct welded $\mathrm{MCO}$ at $2 \mathrm{yr}=1.64 \mathrm{E}+02-\mathbf{3 . 1 5 E}+00=1.61 \mathrm{E}+02 \mathrm{~g}$

Water remaining in 392 direct welded $\mathrm{MCOs}=1.61 \mathrm{E}+02 \mathrm{~g} / \mathrm{MCO} \times 392 \mathrm{MCOs}=6.31 \mathrm{E}+04 \mathrm{~g}=$ $6.31 \mathrm{E}+01 \mathrm{~kg}$

Amount of hydrogen at two yr in $392 \mathrm{MCOs}=2.696 \mathrm{gmol} / \mathrm{MCO}$ x $392 \mathrm{MCOs} \times 2 \mathrm{~g} / \mathrm{gmol} \mathrm{x}$ $0.001 \mathrm{~kg} / \mathrm{g}=2.11 \mathrm{E}+00 \mathrm{~kg}$ 
Pressure of welded MCOs $=\mathrm{nRT} / \mathrm{V}=\left(2.696 \mathrm{gmol} \mathrm{H} \mathrm{g}_{2}+31.0 \mathrm{gmol} \mathrm{He}\right) \times 0.082 \mathrm{~L} \mathrm{~atm} / \mathrm{gmol} \mathrm{K} \mathrm{x}$ $333 \mathrm{~K} / 500 \mathrm{~L}=1.84 \mathrm{~atm}=27.0 \mathrm{psia}=1400$ torr

\subsubsection{C-22 He Gas Supply}

Helium gas is supplied for the following operations: back gas during welding, weld testing, sample cart equipment/hose purging, sample cart pressure decay test, dilution of the MCO internal gas when released to the exhaust system, MCO reinerting (sampling and pressure unit replacement for monitored MCOs), and inerting of the MCO over pack storage tube(s). Assume the following:

- Piping from He supply source to sample/weld station, purge cart station and receiving pit has been initially charged with He to required pressures

- He is supplied at 10 standard $\mathrm{ft}^{3} / \mathrm{hr}$ as back gas during the 4 passes for cap welding and 1 of 2 passes for plate cover welding $(0.5 \mathrm{hr} /$ pass for each weld - private communication with $\mathrm{T}$. A. Delucchi of COGEMA)

- He $=10 \mathrm{ft}^{3} / \mathrm{hr} \times 5$ weld passes $/$ MCO $\times 0.5 \mathrm{hr} /$ weld pass $\times 398 \mathrm{MCOs}=\mathbf{9 , 5 0 0 ~} \mathbf{s t d} \mathbf{f t}^{\mathbf{3}}$

- He is supplied at $10 \mathrm{ft}^{3} / \mathrm{hr}$ for weld testing of the cap and plate cover (private communication with T. A. Delucchi of COGEMA); cover cap void space is estimated at $3.4 \mathrm{ft}^{3}$ - $\mathrm{He}=3.4 \mathrm{ft}^{3} / \mathrm{MCO} \times 4$ circumference weld tests/MCO $\times 398 \mathrm{MCOs}=\mathbf{5 , 4 0 0} \mathbf{s t d ~ f t}^{\mathbf{3}}$

- He is supplied for purging and pressure testing of the MCO sample cart piping, dilution of MCO gas before discharge to the ventilation system (dilute the $\mathrm{H}_{2}$ to 2.0 vol. $\%(<25 \%$ of 8.7 vol. \% flammability limit of $\mathrm{H}_{2}$ in Table B-4 of Piepho and Rittmann (1999)) in order to meet NFPA 69 requirements), reinerting of the monitored MCOs (FDI 1999, FH 1999) and reinerting of monitored MCOs after replacement of the 100 psig pressure sending units. Allow 6,000 std $\mathrm{ft}^{3}$ of helium per MCO sampled ( 8 times) and pressure unit replacement. This results in a total predicted helium usage of $36,000 \mathrm{std} \mathrm{ft}^{3}$ for the monitored $\mathrm{MCO}$ operations.

- Estimated total He usage for normal operations $=9,500+5,400+36,000=50,900 \mathrm{std} \mathrm{ft}^{3} \sim$ 51,000 std $^{3} \mathbf{f t}^{3}=1.44 \mathrm{E}+03 \mathrm{~m}^{3}$

- He is supplied for inerting the over pack storage tube; the inerting of an overpack storage tube is an off normal event and is not included in this PFD calculation.

- The total mass of supply helium is calculated below:

- $\quad$ Mass of He used $=1.44 \mathrm{E}+03 \mathrm{std} \mathrm{m}^{3} \times 1000 \mathrm{~L} / \mathrm{m}^{3} \times 273 \mathrm{~K} / 288 \mathrm{~K}$ x gmol $/ 22.4 \mathrm{~L}$ x 4 $\mathrm{g} / \mathrm{gmol} \times 0.001 \mathrm{~kg} / \mathrm{g}=\mathbf{2 4 4} \mathbf{~ k g}$

\subsubsection{C-23 Monitored MCO Exhaust Gas}

The internal gas of the six monitored MCOs will be sampled eight times during a two-year period. After sampling and before welding, the 100 psi pressure sending and readout units of the monitored MCOs are replaced with a 600 psi pressure sending unit. The MCO gas is exhausted through the sample cart and diluted with helium before entering the building ventilation system. There are no predicted gas constituents at this time except helium and hydrogen. Allow 36,000 std $\mathrm{ft}^{3}$ of helium for sampling six monitored MCOs eight times and pressure unit replacement. Off normal MCO sampling is recovery action and is not included in this estimate. 
- Estimated volume MCO internal gas loss to exhaust system:

- sampling (based on Stream C-12) $=\left[\left(7.56 \mathrm{E}-03 \mathrm{~kg} \mathrm{H}_{2} /(2 \mathrm{~g} / \mathrm{gmol})+2.23 \mathrm{E}-01 \mathrm{~kg} \mathrm{He} /(4\right.\right.$ $\mathrm{g} / \mathrm{gmol})=5.95 \mathrm{E}-02 \mathrm{~kg} \mathrm{~mol}] \times 1000 \mathrm{~g} / \mathrm{kg} \times 22.4 \mathrm{~L} / \mathrm{gmol} \mathrm{x} \mathrm{m}{ }^{3} / 1000 \mathrm{~L} \times 288 \mathrm{~K} / 273 \mathrm{~K}=$ $1.41 \mathrm{E}+01 \mathrm{std} \mathrm{m}^{3}=50 \mathrm{std} \mathrm{ft}^{3}$

- Pressure unit replacement

$$
\frac{(27.0-14.7 \mathrm{psia})(500 \mathrm{~L} / \mathrm{MCO}) \times 6 \mathrm{MCOs}}{(0.082 \mathrm{Latm} / \mathrm{gmol} \times 14.7 \mathrm{psia} / \mathrm{atm} \times 333 \mathrm{~K})} \times \frac{22.4 \mathrm{~L}}{\mathrm{gmol}} \times \frac{288 \mathrm{~K}}{273 \mathrm{~K}} \times \frac{\mathrm{ft}^{3}}{28.3 \mathrm{~L}}=80 \mathrm{std} \mathrm{ft}^{3}
$$

- Total MCO internal gas loss $=50 \mathrm{std} \mathrm{ft}^{3}+80 \mathrm{std} \mathrm{ft}^{3}=130 \mathrm{std} \mathrm{ft}^{3}$

- Mass of He exhausted in MCO internal gas from sampling and pressure unit replacement:

- MCO sampling (see C-12) $=2.23 \mathrm{E}-01 \mathrm{~kg}$

- Pressure sending unit replacement

$\frac{(27.0-14.7 \mathrm{psia})(500 \mathrm{~L} / \mathrm{MCO}) \times 6 \mathrm{MCOs} \times 0.937 \mathrm{He}}{(0.082 \mathrm{~L} \mathrm{~atm} / \mathrm{gmol} \mathrm{K} \times 14.7 \mathrm{psia} / \mathrm{atm} \times 333 \mathrm{~K})} \times 4 \mathrm{~g} / \mathrm{mol} \times 0.001 \mathrm{~kg} / \mathrm{g}=3.44 \mathrm{E}-01 \mathrm{~kg}$

- Internal MCO helium exhausted $=2.23 \mathrm{E}-01 \mathrm{~kg}+3.44 \mathrm{E}-01 \mathrm{~kg}=5.67 \mathrm{E}-01 \mathrm{~kg}$

- Mass of hydrogen exhausted in MCO internal gas:

- $\quad$ MCO sampling (see Stream C-12) $=7.56 \mathrm{E}-03 \mathrm{~kg}$

- Pressure sending unit replacement

$\frac{(27.0-14.7 \mathrm{psia}) \times 500 \mathrm{~L} / \mathrm{MCO} \times 0.063 \mathrm{H}_{2} \times 6 \mathrm{MCOs} \times 2 \mathrm{~g} / \mathrm{gmol} \times 0.001 \mathrm{~kg} / \mathrm{g}}{(0.082 \mathrm{Latm} / \mathrm{gmolK} \times 14.7 \mathrm{psia} / \mathrm{atm} \times 333 \mathrm{~K})}=\mathbf{1 . 1 6 E 0 - 0 2 ~} \mathbf{~ g g}$

- Internal MCO hydrogen exhausted $=7.56 \mathrm{E}-03 \mathrm{~kg}+1.16 \mathrm{E}-02 \mathrm{~kg}=1.92 \mathrm{E}-02 \mathrm{~kg}$

- Total He exhausted during MCO gas sampling and pressure unit replacement $=36,000 \mathrm{std} \mathrm{ft}^{3}$ $=1.02 \mathrm{std} \mathrm{m}^{3}=1.72+01 \mathrm{~kg}$

\subsubsection{C-24 Deleted}

\subsubsection{C-25 Annex Room Air to Weld Station Exhaust}

Annex room air will be the total airflow through the filter system AH-006. Capacity of the AH006 filter is $500 \mathrm{scfm}$ based on FDI (1999a) and H-2-127455, Rev. 2A. Based on $290 \mathrm{~min}$ of weld station usage/MCO (block flow diagram steps 3-16, 3-17, 3-18, 3-24, 3-25, 3-36, 3-40, 341, and 3-42 of H-2-123400, Rev. H)) $160 \mathrm{~min} / \mathrm{MCO}$ for sampling (steps 12-1 through 12-9 of $\mathrm{H}-2-123400, \mathrm{Rev} . \mathrm{H}$ ), and $60 \mathrm{~min} / \mathrm{MCO}$ pressure unit replacement, the weld station exhaust is:

- Weld station exhaust volume $=500 \mathrm{scfm} \times(290 \mathrm{~min} / \mathrm{MCO} \times 398 \mathrm{MCOs}+160 \mathrm{~min} / \mathrm{MCO} \times 48$ $\mathrm{MCOs}+60 \mathrm{~min} / \mathrm{MCO} \times 6 \mathrm{MCOs})=6.17 \mathrm{E}+07 \mathrm{std} \mathrm{ft}^{3}\left(1.74 \mathrm{E}+06 \mathrm{~m}^{3}\right)$.

- Annex room air volume $=6.17 \mathrm{E}+07 \mathrm{ft}^{3}-36,000 \mathrm{ft}^{3} \mathrm{He}\left(\right.$ Stream C-23) $-39,800 \mathrm{ft}^{3} \mathrm{Ar}$ $\left(\right.$ Stream C-16) $=6.16 \mathrm{E}+07 \mathrm{ft}^{3}\left(1.74 \mathrm{E}+06 \mathrm{~m}^{3}\right)$

- Annex room exhaust air mass $=6.16 \mathrm{E}+07 \mathrm{ft}^{3} \times 273 \mathrm{~K} / 288 \mathrm{~K} \times 28.3 \mathrm{~L} / \mathrm{ft}^{3} \times \mathrm{gmol} / 22.4 \mathrm{~L} \mathrm{x}$ $28.97 \mathrm{~g} / \mathrm{gmol} \times 0.001 \mathrm{~kg} / \mathrm{g}=\mathbf{2 . 1 4} \mathrm{E}+06 \mathbf{~ k g}$ 
SNF-5741, Rev. 0

\subsubsection{C-26 Filtered Weld Station Offgas}

The weld station offgas is composed of the annex room air, helium, and argon welding gases.

The total volume is determined by the filter capacity of $500 \mathrm{scfm}$ based on FDI (1999a) and H-2129455 , Rev. 2A, and usage times of $290 \mathrm{~min} / \mathrm{MCO}$ for welding and $160 \mathrm{~min} / \mathrm{MCO}$ for sampling (H-2-123400, Rev. H).

- The weld station exhaust volume $=6.16 \mathrm{E}+07 \mathrm{std}_{\mathrm{ft}}{ }^{3}\left(1.74 \mathrm{E}+06 \mathrm{~m}^{3}\right)$ based on Stream C-25.

- Mass helium exhausted from sampling, pressure unit replacement, and welding $=1.72 \mathrm{E}+02$ $\mathrm{kg}$ (Stream C-23 sampling and pressure unit replacement) $+\left[\left(9500+5400 \mathrm{std}_{\mathrm{ft}}{ }^{3}\right.\right.$ from Stream C-22 welding supply)] $\times 273 \mathrm{~K} / 288 \mathrm{~K} \times 28.3 \mathrm{~L} / \mathrm{ft}^{3} \times \mathrm{gmol} / 22.4 \mathrm{~L}$ x $4 \mathrm{~g} / \mathrm{gmol} \times 0.001$ $\mathrm{kg} / \mathrm{g}=\mathbf{2 . 4 4 E}+02 \mathrm{~kg}$

The total mass is the sum of the air, helium, hydrogen, and argon gases.

- The weld station exhaust mass $=2.14 \mathrm{E}+06 \mathrm{~kg}$ air $($ Stream C-25) $+2.44 \mathrm{E}+02 \mathrm{~kg} \mathrm{He}+7.56 \mathrm{E}-$ $03 \mathrm{~kg} \mathrm{H}_{2}\left(\right.$ Stream C-12) $+1.16 \mathrm{E}-02 \mathrm{~kg} \mathrm{H}_{2}($ Stream C-23) $+1.90 \mathrm{E}+03 \mathrm{~kg} \mathrm{Ar}($ Stream C-16) $=$ $2.14 \mathrm{E}+06 \mathrm{~kg}$

\subsubsection{C-27 Weld Station Spent HEPA Filter Waste}

Weld station filter Stream C-27 contains the AH-006 prefilter and HEPA filter. The dimensions are 12 in $\times 24$ in $\times 2$ in for the prefilter and 12 in x 24 in x 12 in for the HEPA filter based on FDI (1999a). Assume the prefilter weight is $6 \mathrm{lb}$. and the HEPA filter weight is $25 \mathrm{lb}$. The spent filters are assumed to weigh the same as the original filters. At this time there is no information available on change out frequency of the exhaust filter.

\subsubsection{C-28 Replacement Filters for Weld Station}

See Stream C-27 for description.

\subsubsection{C-29 Clean Empty Cask}

The clean empty transport cask is ready to receive a new empty MCO. No mass balance required.

\subsubsection{C-30 Cask Decontamination Material}

Assume decon material of $2 \mathrm{~kg} / \mathrm{MCO}$ and density of $0.6 \mathrm{~kg} / \mathrm{L}$. The amount of the decon material for $398 \mathrm{MCOs}$ is listed below.

- Mass of cask decon material $=2 \mathrm{~kg} / \mathrm{MCO} \times 398 \mathrm{MCO}=796 \mathrm{~kg}$

- Volume of cask decon material $=796 \mathrm{~kg} / 0.6 \mathrm{~kg} / \mathrm{L}=1326 \mathrm{~L}\left(1.33 \mathbf{~ m}^{3}\right)$ 
SNF-5741, Rev. 0

\subsubsection{C-31 Empty Transport Cask}

The empty transport cask is surveyed for contamination before loading with a new, empty MCO. No mass balance required.

\subsubsection{C-32 Cask Decontamination Waste}

Assume there is no change in volume and weights of decon material after use. See Stream C-30 for description.

\subsubsection{C-33 Miscellaneous Decontamination Material}

Assume the 6 monitored MCOs require decontamination material during the 2-year monitoring period and pressure unit replacement. Based on a decon material weight of $2 \mathrm{~kg} / \mathrm{MCO}$ and density of $0.6 \mathrm{~kg} / \mathrm{L}$, the amount of miscellaneous decon material for the monitored MCOs is listed below.

- $\quad$ Mass of monitored MCO decon material $=2 \mathrm{~kg} / \mathrm{MCO}$ x $48(6 \times 8)$ MCO monitoring events $+2 \mathrm{~kg} / \mathrm{MCO} \times 6$ pressure unit replacements $=108 \mathrm{~kg}$

- Volume of monitored MCO decon material $=108 \mathrm{~kg} / 0.6 \mathrm{~kg} / \mathrm{L}=180 \mathrm{~L}\left(\mathbf{0 . 1 8} \mathbf{~ m}^{\mathbf{3}}\right)$

\subsubsection{C-34 Miscellaneous Decontamination Waste}

Assume there is no change in volume and weights of decon material after use. See Stream C-33 for description.

\subsubsection{C-35 Accumulated Solid Waste}

Accumulated solid waste is listed in Table 7.2.7

Table 7.2.7 Accumulated Solid Waste

\begin{tabular}{|l|c|c|c|}
\hline Stream & Mass, $\mathrm{kg}$ & Volume, $\mathrm{m}^{3}$ & Waste Container \\
\hline $\mathrm{C}-19$ & $1,445^{2}$ & 0.33 & 55 gal drum \\
\hline $\mathrm{C}-20$ & 796 & 1.33 & 55 gal drum \\
\hline $\mathrm{C}-32$ & 796 & 1.33 & 55 gal drum \\
\hline C-34 & 108 & 0.18 & 55 gal drum \\
\hline C-51 & 0 & 0 & 55 gal drum \\
\hline Total & $\mathbf{3 , 1 4 5}$ & $\mathbf{3 . 1 7}$ & \\
\hline
\end{tabular}

1. Information on replacement and disposal of this filter is not available at this time. Filter replacement depended on pressure drop and radiation dose rate.

2. Amount includes $20 \mathrm{~kg}$ of plastic weld spool waste. 


\subsubsection{C-36 Packaged Solid Waste}

The solid waste will be packaged into 55-gallon drums or burial boxes. See Stream C-37 for number of drums and Stream C-49 for number of burial boxes. The filled drums and burial boxes are transported to solid waste disposal.

\subsubsection{C-37 Solid Waste Drums}

Table 7.2.8 is based on information in Stream C-35

Table 7.2.8 Solid Waste Disposed in Drums

\begin{tabular}{|c|c|}
\hline Stream & Volume, $\mathrm{m}^{3}$ \\
\hline $\mathrm{C}-19$ & 0.33 \\
\hline $\mathrm{C}-20$ & 1.33 \\
\hline $\mathrm{C}-32$ & 1.33 \\
\hline $\mathrm{C}-34$ & 0.18 \\
\hline C-51 & 0 \\
\hline Total & 3.17 \\
\hline
\end{tabular}

Assume the 55-gallon drums are filled to an $80 \%$ maximum capacity $\left(0.17 \mathrm{~m}^{3}\right)$.

- Minimum number of empty 55 -gallon waste drums $=3.17 \mathrm{~m}^{3} /\left(0.17 \mathrm{~m}^{3} / \mathrm{drum}\right)=19$

\subsubsection{C-38 Cask Service System and Weld Station Exhaust}

The capacity of the cask service offgas system (Stream C-47) is $5 \mathrm{scfm}$ based on design capacity of HEPA exhaust filter FH-2. The design capacity of the weld station exhaust system (Stream C26) is $500 \mathrm{scfm}$. The offgas flowrate is equivalent to Stream C-26 because Stream C-47 is used for off normal conditions. The Stream C-26 information is summarized below.

Table 7.2.9 Cask Service System and Weld Station Exhaust

\begin{tabular}{|c|c|c|c|}
\hline Stream & C-47 & C-26 & Total \\
\hline Air, kg & 0 & $2.14 \mathrm{E}+06$ & $\mathbf{2 . 1 4 E}+\mathbf{0 6}$ \\
\hline Helium, $\mathrm{kg}$ & 0 & $2.44 \mathrm{E}+02$ & $\mathbf{2 . 4 4 E + 0 2}$ \\
\hline Hydrogen, $\mathrm{kg}$ & 0 & $1.92 \mathrm{E}-02$ & $\mathbf{1 . 9 2 E - 0 2}$ \\
\hline Argon., kg & 0 & $1.90 \mathrm{E}+03$ & $\mathbf{1 . 9 0 E}+\mathbf{0 3}$ \\
\hline Total, kg & 0 & $2.06 \mathrm{E}+06$ & $\mathbf{2 . 1 4 E}+\mathbf{0 6}$ \\
\hline
\end{tabular}

\subsubsection{C-39 MHM Ventilation Exhaust}

The air flowrate of the MHM extract system is $800 \mathrm{scfm}$ based on the capacity of MHM exhaust fan (private communication with C. E. Swenson of Fluor Hanford). The design capacity of prefilter PF-1 and HEPA exhaust filter HF-1 is 1,000 scfm. The MHM ventilation system is operated during movement of the MCOs. The following times are required for loading, transfer, and unloading of the MCOs by MHM. 
- $4.0 \mathrm{hr}$ to transfer each monitored MCO (6 total) from transport cask to standard storage tube (steps 2-22 thru 2-27 and 4-1 thru 4-6 of H-2-123400, Rev. H)

- MHM extract system air volume $=4.0 \mathrm{hr} / \mathrm{MCO} \times 60 \mathrm{~min} / \mathrm{hr} \times 800 \mathrm{scfm} \times 6 \mathrm{MCOs}=$ $1.15 \mathrm{E}+06 \mathrm{std} \mathrm{ft}^{3}\left(\mathbf{3 . 2 5} \mathrm{E}+\mathbf{0 4} \mathbf{~ m}^{3}\right)$

- $3.2 \mathrm{hr}$ to transfer each MCO (392 total) from transport cask to sample/weld station (steps 222 thru 2-27 and 3-1 thru 3-4 of H-2-123400, Rev. H)

- MHM extract system air volume $=3.2 \mathrm{hr} / \mathrm{MCO}$ x $60 \mathrm{~min} / \mathrm{hr}$ x $800 \mathrm{scfm}$ x $392 \mathrm{MCOs}$ $=6.02 \mathrm{E}+07 \mathrm{std} \mathrm{ft}^{3}\left(\mathbf{1 . 7 0 ~ E}+06 \mathbf{~ m}^{3}\right)$

- $3.8 \mathrm{hr}$ to transfer each MCO $(392+6 \times 8=440)$ from sample weld station to standard storage tube (steps 12-15 thru 12-19 and 13-1 thru 13-6 of H-2-123400, Rev. H) - $\quad 3.8 \mathrm{hr} \times 60 \mathrm{~min} / \mathrm{hr} \times 800 \mathrm{scfm} \times 440 \mathrm{MCOs}=8.03 \mathrm{E}+07 \mathrm{std} \mathrm{ft}^{3}\left(\mathbf{2 . 2 7} \mathrm{E}+06 \mathbf{~ m}^{3}\right)$

- $3.6 \mathrm{hr}$ to transfer each monitored MCO $(6 \times 8=48)$ from standard storage tube to sample weld/station for sampling and welding after $8^{\text {th }}$ sample (steps 14-3 thru 14-10 and 3-1 thru 3-4 of H-2-123400, Rev. H)

- $\quad 3.6 \mathrm{hr} / \mathrm{MCO} \times 60 \mathrm{~min} / \mathrm{hr} \times 800 \mathrm{scfm} \times 48 \mathrm{MCOs}=8.29 \mathrm{E}+06 \mathrm{std} \mathrm{ft}^{3}\left(\mathbf{2 . 3 4 E}+05 \mathbf{~ m}^{3}\right)$

- $5.25 \mathrm{hr}$ to transfer and place each intermediate impact absorber in the standard storage tubes (steps 9-3 thru 9-18 of H-2-123400, Rev. H)

- $5.25 \mathrm{hr} / \mathrm{MCO} \times 60 \mathrm{~min} / \mathrm{hr} \times 800 \mathrm{scfm} \times 198$ intermediate impact absorbers $=4.99 \mathrm{E}+07$ std $\mathrm{ft}^{3}\left(1.41 \mathrm{E}+06 \mathrm{~m}^{3}\right)$

- Total volume air exhausted by MHM extract system during MCO transfers $=\mathbf{1 . 9 9 E}+08$ $\operatorname{std~ft}^{3}\left(5.62 \mathrm{E}+06 \mathrm{~m}^{3}\right)$

- Total mass air exhausted by MHM extract system during MCO transfers $=1.99 \mathrm{E}+08 \mathrm{std} \mathrm{ft}^{3}$ x $28.3 \mathrm{~L} / \mathrm{ft}^{3} \times 273 \mathrm{~K} / 288 \mathrm{~K} \mathrm{x} \mathrm{gmol} / 22.4 \mathrm{~L}$ x $28.9 \mathrm{~g} / \mathrm{gmol}$ x $0.001 \mathrm{~kg} / \mathrm{g}=\mathbf{6 . 8 9 E}+06 \mathbf{~ k g}$

\subsubsection{C-40 Replacement Filters for MHM}

The MHM extract filter system PF-1/HF-1 consists of one prefilter and one high efficiency filter. The PF-1 filter is 12 in $x 24$ in $x 2$ in and weighs and estimated $6 \mathrm{lb}$. The HF-1 filter is 12 in $\mathrm{x}$ 24 in $x 11.5$ in and weighs $40 \mathrm{lb}$ (private communication with C. E. Swenson of Fluor Hanford). There is no information available at this time on change out frequency of the MHM filters.

\subsubsection{C-41 Spent Filters From MHM}

There are no changes in the filter mass and volume of the MHM extract system filters. See Stream C-40 for description.

\subsubsection{C-42 Operating Area Ventilation Exhaust}

The total volume of gas exhausted to the stack is determined by the design capacity of the PF001/002 filters. Each filter has a capacity of 9,000 scfm (Flanders 1997 and H-2-129580, Rev. 3 ). Based on a two year-operating period, the amount of gas exhausted to the environment is listed below. 
Table 7.2.10 Ventilation System Exhaust

\begin{tabular}{|c|c|c|c|c|}
\hline Stream & C-38 & C-39 & Other & Total \\
\hline Air, $\mathrm{kg}$ & $2.14 \mathrm{E}+06$ & $6.89 \mathrm{E}+06$ & $\mathbf{3 . 1 8 E}+\mathbf{0 8}$ & $\mathbf{3 . 2 7} \mathrm{E}+\mathbf{0 8}$ \\
\hline $\mathrm{He}, \mathrm{kg}$ & $2.44 \mathrm{E}+02$ & 0 & 0 & $2.44 \mathrm{E}+02$ \\
\hline $\mathrm{H}_{2}, \mathrm{~kg}$ & $1.92 \mathrm{E}-02$ & 0 & 0 & $1.92 \mathrm{E}-02$ \\
\hline $\mathrm{Ar}, \mathrm{kg}$ & $1.90 \mathrm{E}+03$ & 0 & 0 & $1.90 \mathrm{E}+03$ \\
\hline Total & $2.14 \mathrm{E}+06$ & $\mathbf{6 . 8 9 E}+\mathbf{0 6}$ & $\mathbf{3 . 1 8 E}+\mathbf{0 8}$ & $\mathbf{3 . 2 7} \mathbf{E}+\mathbf{0 8}$ \\
\hline
\end{tabular}

- Volume gas discharged to environment based on 2 yr operation $=9,000 \mathrm{scfm} \times 2 \mathrm{yr} \times 365$ day/yr x $24 \mathrm{hr} /$ day $\times 60 \mathrm{~min} / \mathrm{hr}=9.46 \mathrm{E}+09 \mathrm{std} \mathrm{ft}^{3}$ air $\left(2.68 \mathrm{E}+08 \mathrm{~m}^{3}\right)$

- Mass of gas discharged to environment based on $2 \mathrm{yr}$ operation $=9.46 \mathrm{E} 09 \mathrm{std}^{3} \mathrm{ft}^{3}$ air $\times 28.3$ $\mathrm{L} / \mathrm{ft}^{3} \times 273 \mathrm{~K} / 288 \mathrm{~K} \mathrm{gmol} / 22.4 \mathrm{~L} \times 28.9 \mathrm{~g} / \mathrm{gmol} \times 0.001 \mathrm{~kg} / \mathrm{g}=\mathbf{3 . 2 7 \mathrm { E }}+\mathbf{0 8} \mathbf{~ k g}$

- Mass of other air from operating area and support building based on $2 \mathrm{yr}$ operation $=3.27 \mathrm{E}+08$ $\mathrm{kg}-2.14 \mathrm{E}+06-6.89 \mathrm{E}+06 \mathrm{~kg}=\mathbf{3 . 1 8 E}+\mathbf{0 8} \mathbf{~ k g}$

\subsubsection{C-43 Spent HEPA Filter From Operating Area}

There is no change in mass or volume of the operating vault filters. See Stream C-44 for description.

\subsubsection{C-44 Replacement HEPA Filter for Operating Area}

Filter system PF-001/002 each consists of nine prefilters and nine HEPA filters based on Flanders (1997). The size of the prefilters is 24 in $\times 24$ in $\times 2$ in. The size of the HEPA filters is 24 in $\times 24$ in x 11.5 in. Assume prefilters weigh $12 \mathrm{lb}$ each and HEPA filters weigh $40 \mathrm{lb}$ each. The design capacity is $9500 \mathrm{scfm}$ and normal operation is $9,000 \mathrm{scfm}$. There is no information available at this time on change frequency out of the operating vault filters.

\subsubsection{C-45 Spent HEPA Filters From Support Building}

There is no information available at this time on change out of these filters. See Stream C-46 for description.

\subsubsection{C-46 Replacement HEPA Filters for Support Building}

Filter system AH-004 consists of one prefilter and one HEPA filter. The prefilter is 24 in $x 24$ in x 2 in and the HEPA filter is 24 in $x 24$ in x 11.5 in based on DE\&S (1997). Assume the prefilter weighs $12 \mathrm{lb}$ and the HEPA filter weighs $40 \mathrm{lb}$. The filter design capacity is $920 \mathrm{scfm}$ (H-2-129587, Rev. 3).

\subsubsection{C-47 Cask Service Exhaust System}

Cask servicing is an off normal condition and there is no predicted exhaust air or waste. The FH-2 HEPA filter design capacity is $5 \mathrm{scfm}$. The filter weights $66 \mathrm{lb}$ and measures 28 in flange to flange based on Fairey (1999a). 


\subsubsection{C-48 Solid Waste Burial Boxes}

The waste boxes are used for disposal of the filters from Streams C-11, C-27, C-41, C-43 and C45. Information on replacement of these filters, size of the disposal boxes, and number of burial boxes is not available at this time. See Stream C-49.

\subsubsection{C-49 Accumulated Filter Waste}

Information on replacement of the exhaust filters for these streams is not available at this time. Filter replacement depended on pressure drop and radiation dose rate.

Table 7.2.11. Burial Box Waste

\begin{tabular}{|c|c|c|c|}
\hline Stream & Mass, $\mathrm{kg}$ & Volume, $\mathrm{m}^{3}$ & Waste Container \\
\hline $\mathrm{C}-11$ & 0 & 0 & Burial box \\
\hline $\mathrm{C}-27$ & 0 & 0 & Burial box \\
\hline C-41 & 0 & 0 & Burial box \\
\hline C-43 & 0 & 0 & Burial Box \\
\hline C-45 & 0 & 0 & Burial box \\
\hline Total & 0 & 0 & \\
\hline
\end{tabular}

\subsubsection{C-50 Replacement Sample HEPA Filter}

The FH-9 sample HEPA filter for MCO gas sampling is 6.6 in outside diameter by 12 in long and weights an estimated $29 \mathrm{lb}$ based on Fairey (1999). There is no information available at this time on change out frequency of this filter. The design capacity is $33 \mathrm{scfm}$.

\subsubsection{C-51 Spent HEPA Filter from Sample Hood}

There are no changes in the filter mass and volume of the sample HEPA filter. See Stream C-50 for description.

\subsubsection{C-52 Helium Reinerted Monitored MCOs to Welding}

The monitored MCOs are vented, purged with helium, low range pressure sending unit replaced, reinerted with helium to the same pressure, and leak checked before welding. The MCO gas is diluted with helium and vented to the sample hood exhaust through the sample cart. 
SNF-5741, Rev. 0

Table 7.2.12 Stream C-52 (After Pressure Sending/Readout Unit Replacement)

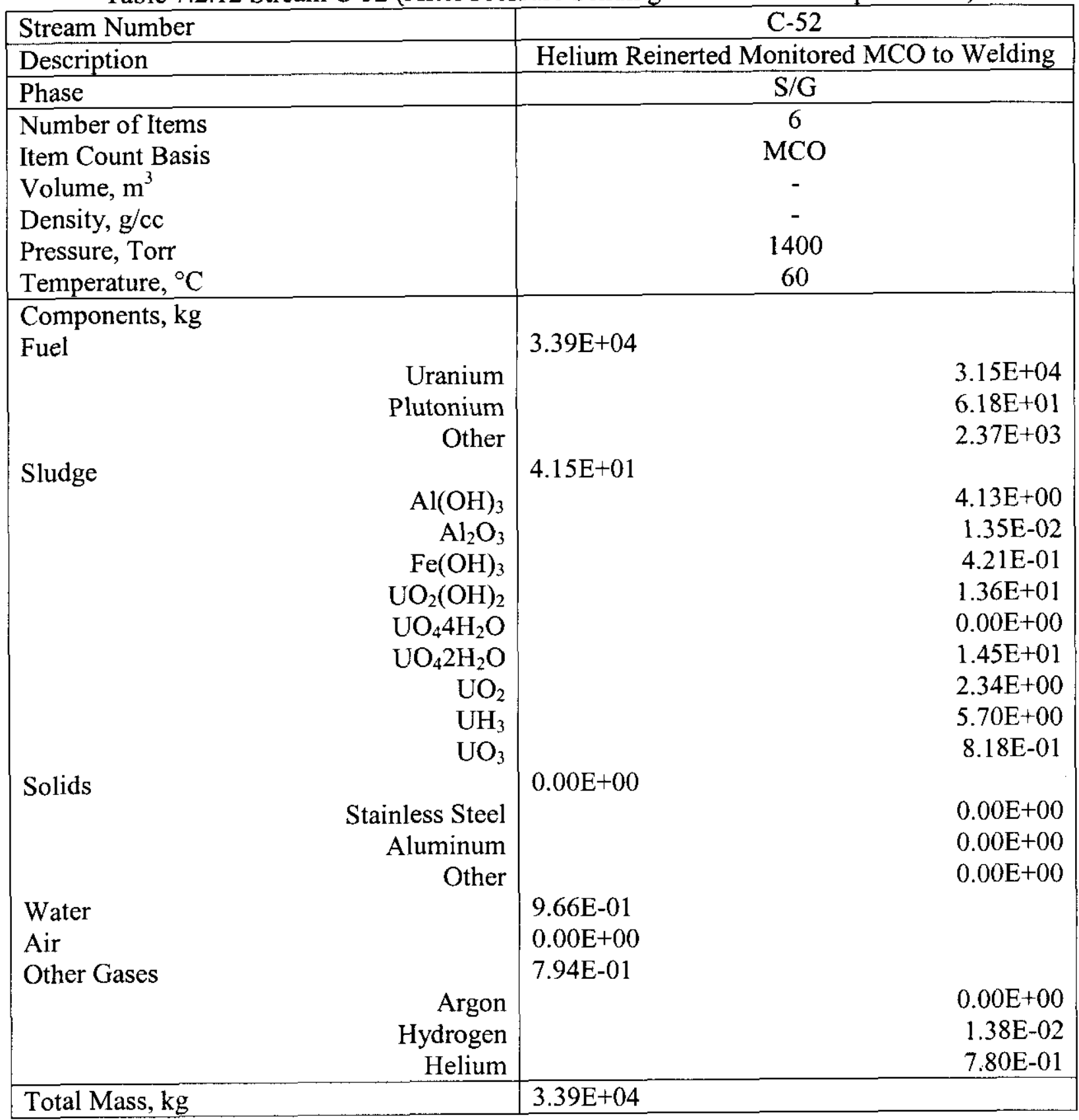

Mass $\mathrm{H}_{2}$ and $\mathrm{He}$ inside monitored MCOs after venting for pressure unit replacement:

$$
\begin{aligned}
& H_{2} \text { mass }=\frac{1 \mathrm{~atm} \times 500 \mathrm{~L} / \mathrm{MCO} \times 6 \mathrm{MCOs} \times 0.063 \mathrm{H}_{2}}{(0.082 \mathrm{Latm} / \mathrm{gmol} \mathrm{K} \times 333 \mathrm{~K})}=6.92 \mathrm{E}+00 \mathrm{gmol}=1.38 \mathrm{E}-02 \mathrm{~kg} \\
& \mathrm{He} \text { mass }=\frac{1 \mathrm{~atm} \times 500 \mathrm{~L} / \mathrm{MCO} \times 6 \mathrm{MCOs} \times 0.937 \mathrm{He}}{(0.082 \mathrm{Latm} / \mathrm{gmol} \mathrm{K} \times 333 \mathrm{~K})}=1.03 \mathrm{E}+02 \mathrm{gmol}
\end{aligned}
$$


Mass He inside monitored MCOs after He reinerting for pressure unit replacement:

$$
27.0 \mathrm{psia}=\frac{\left(1.10 \mathrm{E}+02 \mathrm{gmol} \mathrm{H}_{2} \& \mathrm{He}+\mathrm{He} \text { added }\right) \times 0.082 \mathrm{Latm} / \mathrm{gmol} \mathrm{K} \times 333 \mathrm{~K}}{(500 \mathrm{~L} / \mathrm{MOCO} \times 6 \mathrm{MCOs} \times \mathrm{atm} / 14.7 \mathrm{psia})}
$$

Mass $\mathrm{He}$ added for reinerting $=9.18 \mathrm{E}+01$ gmol

Total He mass $=1.03 \mathrm{E}+02 \mathrm{gmol}+9.18 \mathrm{E}+01 \mathrm{gmol}=1.95 \mathrm{E}+02 \mathrm{gmol}=7.8 \mathrm{E}-01 \mathrm{~kg}$

\subsubsection{C-53 MCO Pressure Units}

A supply of 600 psi pressure sending units for the monitored MCOs and 600 psi pressure readout units for all welded MCOs will be stored at the warehouse and delivered to the CSB by truck. There is no change of material balance inside the MCO. Pressure unit size and weight are based on Douglas and Ohl (1999) and private communication with D. G. Douglas of Vista Research, Inc.

- 600 psi sending units for six monitored MCOs

- Approximate Volume $=6 \mathrm{MCOs} \times(3.0 \mathrm{dia} \times 2.0 \mathrm{in} \mathrm{h}) / \mathrm{unit} \times 1 \mathrm{unit} / \mathrm{MCO}=$ $8.48 \mathrm{E}+01 \mathrm{in}^{3}$

- $\quad$ Weight $=6 \mathrm{MCOs} \times 2.19 \mathrm{lb} /$ unit $\times 1 \mathrm{unit} / \mathrm{MCO}=1.31 \mathrm{E}+01 \mathrm{lb}$

- 600 psi readout units for all $398 \mathrm{MCOs}$

- Approximate Volume $=398 \mathrm{MCOs} \times(2.7$ in dia $\times 0.937$ in $\mathrm{h}) /$ unit $\times 1$ unit/MCO $=$ $2.13 \mathrm{E}+03 \mathrm{in}^{3}$

- $\quad$ Weight $=398 \mathrm{MCOs} \times 0.38 \mathrm{lb} /$ unit $\times 1$ unit $/ \mathrm{MCO}=1.51 \mathrm{E}+02 \mathrm{lb}$

Total volume $=8.48 \mathrm{E}+01 \mathrm{in}^{3}+2.13 \mathrm{E}+03 \mathrm{in}^{3}=2.21 \mathrm{E}+03 \mathrm{in}^{3}=3.63 \mathrm{E}-02 \mathrm{~m}^{3}$

Total weight $=1.31 \mathrm{E}+01 \mathrm{lb}+1.51 \mathrm{E}+02 \mathrm{lb}=1.64 \mathrm{E}+02 \mathrm{lb}=7.44 \mathrm{E}+01 \mathrm{~kg}$

\subsubsection{IC-083 Filtered and Monitored Exhaust From CSB}

See Stream C-42 for description

\subsubsection{IC-085 CSB Support Solid Waste}

See Stream C-36 for description.

\subsubsection{IC-087 New MCO Shell for Cask Loading}

A small supply of new, empty MCOs in shipping crates will be delivered by truck from the warehouse to CSB. These MCOs will be unpacked and stored at the CSB until loaded into an empty transport cask and transported to the $\mathrm{K}$ Basins. The empty shipping crates are returned to the vendor. Size and weight of the new, empty MCOs are based on Drawing H-2-828041, Rev. 4 , and private communication with L. H. Goldmann of Fluor Hanford. 
Approximate (storage) volume $=24$ in dia $x 157.4$ in $\mathrm{h}=7.12 \mathrm{E}+04 \mathrm{in}^{3}$ Average measured weight $=1942 \mathrm{lb}$

Total volume $=398 \mathrm{MCOs} \times 1.72 \mathrm{E}+04 \mathrm{in}^{3} / \mathrm{MCO}=2.83 \mathrm{E}+07 \mathrm{in}^{3}=4.64 \mathrm{E}+02 \mathrm{~m}^{3}$

Total weight $=398 \mathrm{MCOs} \times 1942 \mathrm{lb} / \mathrm{MCO}=7.73 \mathrm{E}+05 \mathrm{lb}=3.50 \mathrm{E}+05 \mathrm{~kg}$

\subsubsection{IC-129 Liquid Effluents}

Condensate from the compressed air and ventilation systems is collected in the SU-1 and SU-2 sump and loaded into a 55 gallon drum for sampling and disposal. Contaminated condensate is transported to the LERF for disposal. Non contaminated condensate is discharged to the ground in accordance with SNF Project Process Standard 409 for maintenance type discharges.

The AH-001 and AH-002 fans remain in operation continuously for operating area return air temperatures $(\mathrm{TE}-265)$ between $63^{\circ} \mathrm{F}$ and $82^{\circ} \mathrm{F}$. When the return air temperature increases to $82^{\circ} \mathrm{F}$, condensing unit CU-001 (evaporator temperature $=52^{\circ} \mathrm{F}$ ) will start and remain in operation. If the return air temperature increases to $85^{\circ} \mathrm{F}$, condensing unit CU-002 (evaporator temperature $=52^{\circ} \mathrm{F}$ ) will start and remain in operation in order to hold the return air temperature constant. As the cooling load decreases, the operation of CU-002 and CU-001 ceases (Notes on H-2-129582, Rev. 3). Table 7.2.13 lists the monthly average psychrometric data between 1950 and 1997 (Hoitink and Burk 1998). The months of July and August have the highest dry and wet bulb temperatures. The monthly average dew point temperatures are less than the $52^{\circ} \mathrm{F}$ evaporator temperature of CU-001 and CU-002. Therefore, no HVAC condensate is anticipated based on monthly average data.

Table 7.2.13 Monthly Average Psychrometric Data

\begin{tabular}{|l|l|l|l|l|l|l|l|l|l|l|l|l|l|}
\hline Category & Jan & Feb & Mar & Apr & May & Jun & Jul & Aug & Sep & Oct & Nov & Dec & Annual \\
\hline Dry bulb & 30.9 & 37.4 & 45.1 & 53.2 & 62.2 & 69.9 & 77.2 & 75.6 & 66.4 & 53.0 & 40.0 & 32.4 & $53.6^{\circ} \mathrm{F}$ \\
\hline Wet bulb & 28 & 34 & 38 & 44 & 50 & 55 & 58 & 58 & 53 & 45 & 36 & 30 & $44^{\circ} \mathrm{F}$ \\
\hline Rel. hum. & 77.3 & 70.5 & 56.6 & 47.3 & 42.8 & 39.6 & 33.3 & 35.6 & 42.2 & 56.4 & 73.6 & 80.3 & $54.6^{\circ}$ \\
\hline Dew Point & 24.2 & 27.6 & 28.8 & 31.6 & 36.9 & 41.5 & 43.6 & 43.9 & 40.4 & 36.1 & 31.3 & 26.5 & $34.4^{\circ} \mathrm{F}$ \\
\hline
\end{tabular}

Additional data for extremes of monthly average psychrometric data show the highest dry bulb temperatures were $83.3^{\circ} \mathrm{F}$ in July 1985 and $82.5^{\circ} \mathrm{F}$ in August 1967. The highest wet bulb temperatures were $62^{\circ} \mathrm{F}$ in July 1985 and $61^{\circ} \mathrm{F}$ in August 1991.

Based on July 1985 extreme averages and an air handler evaporator temperature of $52^{\circ} \mathrm{F}$, cooling of the operating area air would increase the relative humidity from a value of about $31 \%$ to near $100 \%$. The moisture in the air would remain at about $0.008 \mathrm{lb}$ water/lb dry air producing very little HVAC condensate at the extreme average conditions.

Information on compressed air requirements and resulting condensate is based on an average flowrate of $40 \mathrm{scfm}$ (FDI 1996) and monthly average psychrometric data. The results are summarized in Table 7.2.14. 
SNF-5741, Rev. 0

Table 7.2.14 Estimated Compressed Air Condensate

\begin{tabular}{|l|l|l|l|l|l|l|l|l|l|l|l|l|l|}
\hline Category & Jan & Feb & Mar & Apr & May & Jun & Jul & Aug & Sep & Oct & Nov & Dec & Annual \\
\hline Dry bulb & 30.9 & 37.4 & 45.1 & 53.2 & 62.2 & 69.9 & 77.2 & 75.6 & 66.4 & 53.0 & 40.0 & 32.4 & $53.6^{\circ} \mathrm{F}$ \\
\hline Dry air vol. $\mathrm{ft}^{3} / \mathrm{lb}$ & 12.4 & 12.6 & 12.7 & 13.0 & 13.2 & 13.4 & 13.6 & 13.5 & 13.3 & 13.0 & 12.6 & 12.4 & - \\
\hline Dew Point & 24.2 & 27.6 & 28.8 & 31.6 & 36.9 & 41.5 & 43.6 & 43.9 & 40.4 & 36.1 & 31.3 & 26.5 & $34.4^{\circ} \mathrm{F}$ \\
\hline $\mathrm{lb} \mathrm{H}_{2} \mathrm{O} / \mathrm{lb}$ dry air $10^{-3}$ & 2.64 & 3.11 & 3.28 & 3.72 & 4.61 & 5.53 & 6.45 & 6.06 & 5.13 & 4.43 & 3.67 & 2.94 & - \\
\hline $\mathrm{Avg} \mathrm{H}_{2} \mathrm{O} 10^{-4} \mathrm{lb} / \mathrm{ft}^{3}$ & 2.13 & 2.47 & 2.58 & 2.86 & 3.49 & 4.12 & 4.74 & 4.49 & 3.86 & 3.41 & 2.91 & 2.37 & - \\
\hline $\begin{array}{l}\mathrm{H}_{2} \mathrm{O} \text { into compressor } \\
10^{2} \mathrm{lb}\end{array}$ & 3.80 & 3.98 & 4.60 & 4.94 & 6.23 & 7.12 & 8.46 & 8.02 & 6.67 & 6.08 & 5.03 & 4.23 & 74.19 \\
\hline
\end{tabular}

Dry air volume $=\mathrm{V} / \mathrm{n}=\mathrm{RT} / \mathrm{P} \mathrm{ft}^{3} / \mathrm{lb}$

$\mathrm{Lb} \mathrm{H}_{2} \mathrm{O} / \mathrm{lb}$ dry air is estimated from thermodynamic properties of moist air at standard atmospheric pressure (Perry 1984) and the dew point temperature.

Average $\mathrm{H}_{2} \mathrm{O} \mathrm{lb} / \mathrm{ft}^{3}=\mathrm{lb} \mathrm{H}_{2} \mathrm{O} / \mathrm{lb}$ dry air/dry air volume $\mathrm{ft}^{3} / \mathrm{lb}$

$\mathrm{H}_{2} \mathrm{O}$ into compressor/month (lb) = average $\mathrm{H}_{2} \mathrm{O} \mathrm{lb} / \mathrm{ft}^{3} \times 40 \mathrm{scfm} \times 60 \mathrm{~min} / \mathrm{hr} \times 24 \mathrm{hr} /$ day $\times$ no. days/month

Annual average condensate from air compressor operation is estimated as $7.42 \mathrm{E}+03 \mathrm{lb} / \mathrm{yr}$.

Mass of condensate based on $2 \mathrm{yr}$ of CSB operation $=2 \mathrm{yr} \times 7.42 \mathrm{E}+03 \mathrm{lb} / \mathrm{yr}=1.48 \mathrm{E}+04 \mathrm{lb}=$ $6.74 \mathrm{E}+03 \mathrm{~kg}$

Volume of condensate $6.74 \mathrm{E}+04 \mathrm{~kg} / 0.998 \mathrm{~kg} / \mathrm{L} \times 0.001 \mathrm{~m}^{3} / \mathrm{L}=\mathbf{6 . 7 5 E}+00 \mathrm{~m}^{3}$

Number of 55 gallon drums at $80 \%$ capacity $=6.75 \mathrm{~m}^{3} /(55 \mathrm{gal} \times 0.8 \times 3.78 \mathrm{~L} / \mathrm{gal}) \times 1000 \mathrm{~L} / \mathrm{m}^{3}$ $=41$

\subsubsection{IC-233 MCO Cover Cap, Plug, and Plate}

A supply of cover caps, test plugs, and cover plates will be stored at the warehouse and delivered to the CSB by truck. The addition of these components do not change the material balance inside the MCO. Size and weight of the components are based on Drawing H-2-828042, Rev. 4, and private communication with L. H. Goldmann of Fluor Hanford.

\section{- Canister Cover Cap}

- Approximate (storage) volume $=25.3$ in dia $\times 9.0$ in $\mathrm{h}=4.52 \mathrm{E}+03 \mathrm{in}^{3}$

- Average measured weight $=500 \mathrm{lb}$

- Cover Plate

- Approximate volume $=4.0$ in dia $\times 0.38$ in $\mathrm{h}=4.8 \mathrm{E}+00 \mathrm{in}^{3}$

- Average measured weight $=1.0 \mathrm{lb}$ 
- Test Plug

- Approximate volume $=2.38$ in $\operatorname{dia} \times 2.75 \mathrm{in} \mathrm{h}=1.22 \mathrm{E}+01 \mathrm{in}^{3}$

- Average measured weight $=2.0 \mathrm{lb}$

Total Volume $=398 \mathrm{MCOs} x\left(4.52 \mathrm{E}+03 \mathrm{in}^{3}+4.8 \mathrm{E}+00 \mathrm{in}^{3}+1.22 \mathrm{E}+01 \mathrm{in}^{3}\right)=1.81 \mathrm{E}+06 \mathrm{in}^{3}$ $=2.96 \mathrm{E} 01 \mathrm{~m}^{3}$

Total Weight $=398 \mathrm{MCOs} x(500 \mathrm{lb}+1.0 \mathrm{lb}+2.0 \mathrm{lb})=2.00 \mathrm{E}+05 \mathrm{lb}=9.08 \mathrm{E}+04 \mathrm{~kg}$

\subsection{RESULTS}

Results of the calculations are shown in Appendix A and drawing H-2-825869, Rev. 2.

\subsection{CONCLUSIONS}

Drawing H-2-825869, Rev. 2 reflects the average design capacity and current processing strategy.

\subsection{REFERENCES}

DE\&S, 1997, Contract No. KH-5511-W-379, Canister Storage Building Government Furnished Equipment Data Transmittal, letter MCC-5511-1262, Duke Engineering \& Services Hanford, Inc., Richland, Washington.

Douglas, D. G. and P.C. Ohl, 1999, Magnetically Coupled Pressure Gauge: Definitive Design, TM-FO21-99-05, Vista Research, Inc., Richland, Washington.

Duncan, D. R., and M. G. Plys*, 1998, MCO Internal Gas Composition and Pressure During Interim Storage, HNF-SD-SD-TI-040, Rev. 3, Duke Engineering and Services Hanford Inc., Richland, Washington and *Fauske and Associates, Burr Ridge, Illinois.

Ellis and Watts, 1999, Installation Operation and Maintenance Manual for Exhaust Unit CSBEF-005, K0797-08, Rev. 0, Ellis and Watts, Batavia, Ohio.

Fairey, 1999, DE\&S Hanford Data Book, MO98666, Fairey Microfiltrex, Fareham, England.

Fairey, 1999a, DE\&S Hanford Data Book, MO98678, Fairey Microfiltrex, Fareham, England.

FDI, 1999, System Design Description, MCO Sampling/Welding (MSW) System, Fluor Daniel Inc., Richland, Washington.

FDI, 1999a, MCO Sampling/Weld Station Exhaust Unit, W-379-C-CSB-15835, Fluor Daniel Inc., Richland, Washington.

FDI 1996, Condensate from Instrument Air Compressor (process note to file), Fluor Daniel Inc, Richland, Washington. 
FH 1999, Spent Nuclear Fuel Project Final Safety Analysis Report Annex A "Canister Storage Building Final Safety Analysis Report” HNF-3353, Rev. 0, Fluor Hanford, Richland, Washington.

Flanders, 1997, Technical Manual, Purchase Order MBS-SDX-A18981, Job 96508, Flanders/Charcoal Service Corporation, Bath, North Carolina.

Goldmann, L. H., 2000, Performance Specification for the Spent Nuclear Fuel Multi-Canister Overpack, HNF-S-0426, Rev. 6, fluor Hanford, Richland, Washington.

Hoitink, D. J. and K. W. Burk, 1998, Hanford Site Climatological Data Summary 1997, With Historical Data, PNNL-17794, Pacific Northwest National Laboratory, Richland, Washington.

Klem, M. J.*, and A. L. Pajunen, 2000, Spent Nuclear Fuel Project Cask/Transportation Process Flow Diagram Mass Balance Calculation, SNF-5046, Rev. 0, Fluor Hanford, *Cogema Engineering Corporation, Richland, Washington.

NFPA 69,1997, Standard for Explosion Prevention Systems, National Fire Protection Association, Quincy, Massachusetts.

Piepho, M. R and P. R. Rittmann, 1999, Cold Vacuum Drying Facility Design Basis Accident Analysis Documentation, HNF-2770, Rev. 3, Fluor Daniel Northwest Inc, Richland, Washington.

Reed, A. V and A. L. Pajunen*, 1999, Evaluation of Canister Storage Building Cask Receipt Pressure, HNF-4509, Rev. 0, COGEMA Engineering Corporation Richland, Washington and *Numatec Hanford Corporation Richland, Washington.

Rielly, M. A, 1998, Spent Nuclear Project Technical Data Book, HNF-SD-SNF-TI-015, Rev. 6, Duke Engineering and Services Hanford Inc., Richland, Washington.

Pajunen, A. L., 2000, Spent Nuclear Fuel Project Product Specification, HNF-SD-SNF-OCD-001, Rev. 4, Fluor Hanford, Richland, Washington.

Perry, R. H. and D. W. Green, 1984 “Perry's Chemical Engineer's Handbook" Sixth Edition, R. H. Perry and D. W. Green, editors McGraw Hill Inc., New York, New York.

Sherrell, D. L, 1999, Multi-canister Overpack Combustible Gas Management Leak Detection Criteria, HNF-2155, Rev. 1 Fluor Hanford, Richland, Washington.

Smith, K. E., 1999, MCO Design Report, HNF-SD-SNF-DR-003, Rev. 2, Fluor Daniel Hanford Inc., Richland, Washington. 
SNF Standard 409, 2000, Discharges to Ground SNF Project Process Standards, Rev. 1, Fluor Hanford, Richland, Washington.

H-2-123400, Rev. H, Operational Sequence Block Flow Diagram.

H-2-129582, Rev. 3, P \& ID HVAC Operating Area CSB-AH-001.

H-1-81168, Rev. 3, SNF Project Cask/Transportation Level 1 Process Flow Diagram.

H-2-825869, Rev. 2, SNF Project Canister Storage Building Level 1 PFD.

H-2-828041, Rev. 4, Multi-Canister Overpack Assembly

H-2-828042, Rev. 4, MCO Mechanical Closure

H-2-828048, Rev. 3 MCO Process Port Cover Plates.

H-2-129455, Rev. 2A, HVAC MCO Sampling/Weld Station Flow Diagrams.

H-2-129580, Rev. 3, HVAC Composite Diagram

H-2-129587, Rev. 3, P\&ID HVAC Support Area Equipment 
SNF-5741, Rev. 0

\section{APPENDIX A}

MS Excel Spreadsheet Calculations

For Development

Of Drawing H-2-825869, Rev. 2 


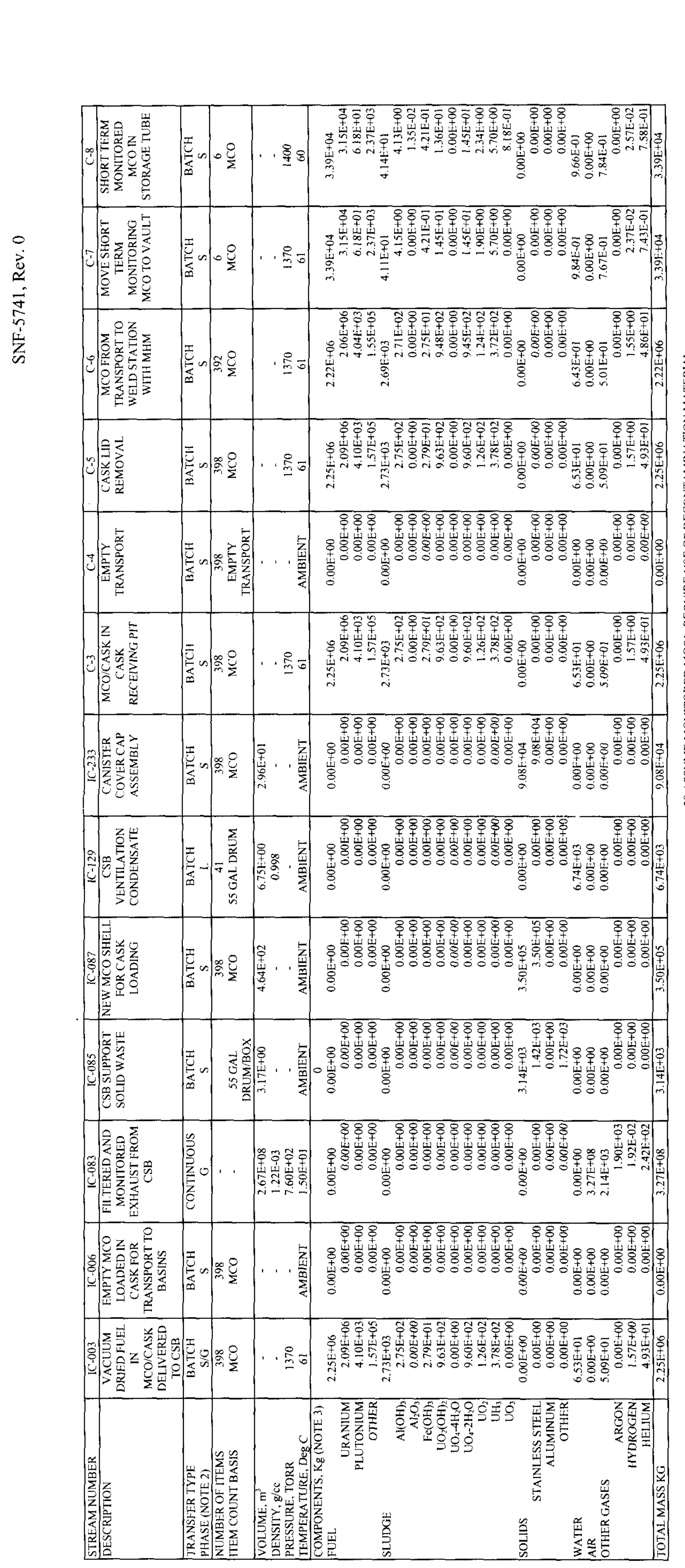
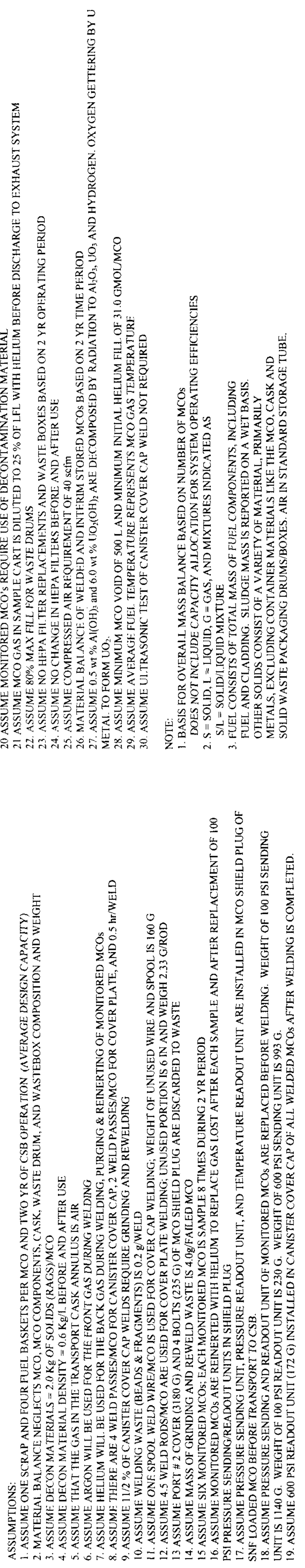


\begin{tabular}{|c|c|c|c|c|}
\hline 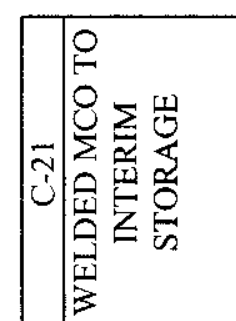 & $\left|\begin{array}{l}\mathbb{E} \\
E \\
\infty \\
\infty\end{array}\right|$ & & $\frac{8}{9} 8$ & 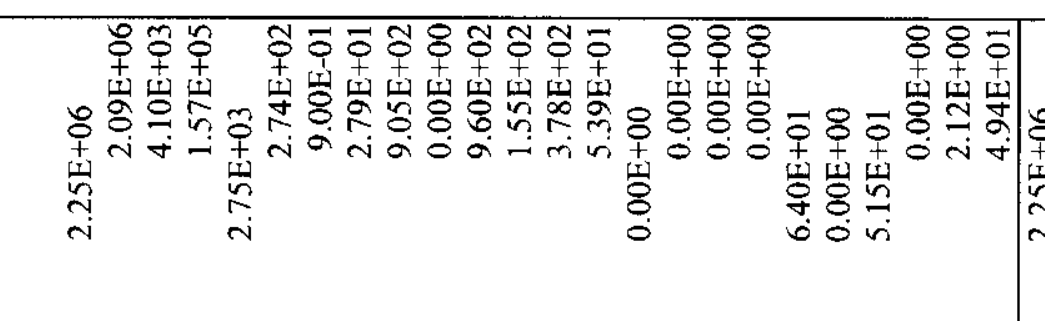 \\
\hline $\begin{array}{ll} \\
0 \\
0\end{array}$ & 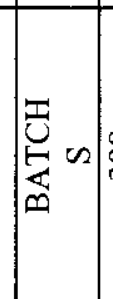 & &.$\approx$ & 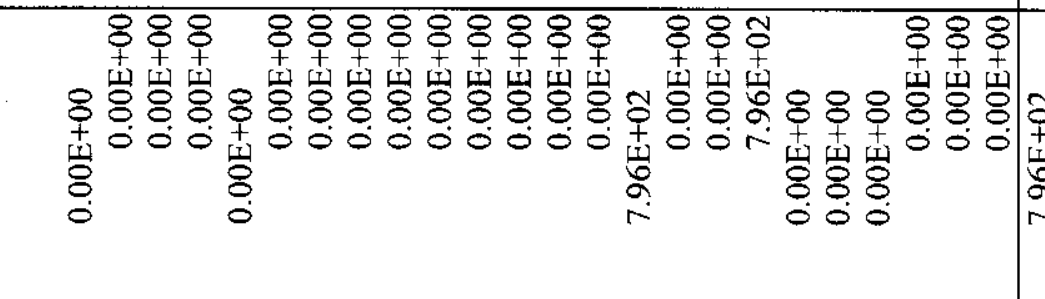 \\
\hline 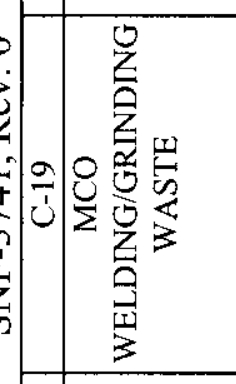 & & & 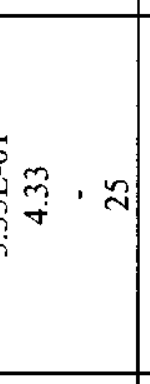 & 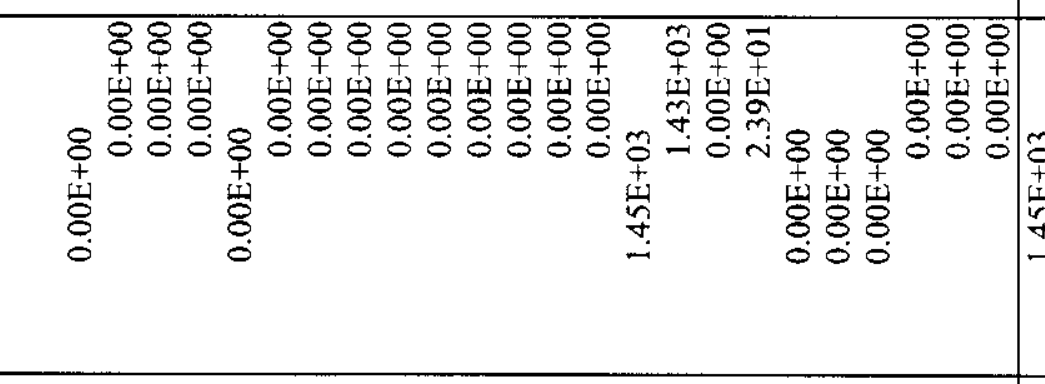 \\
\hline 完) & & & & \\
\hline 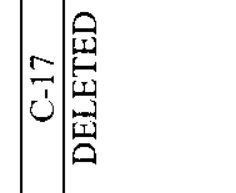 & & & & \\
\hline 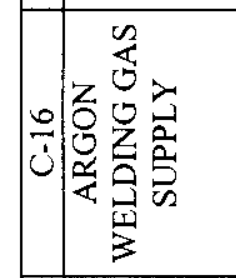 & O & & $=$ & 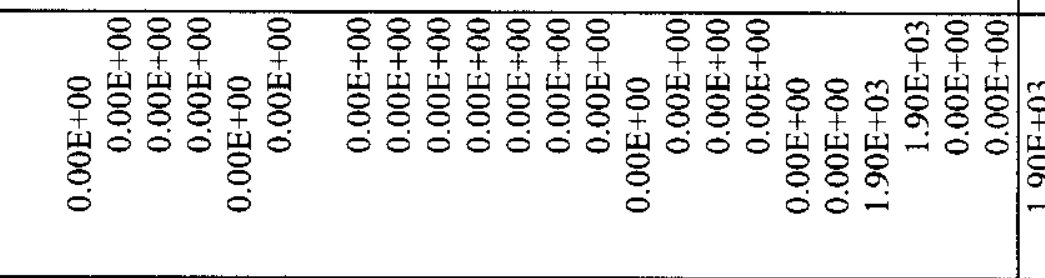 \\
\hline 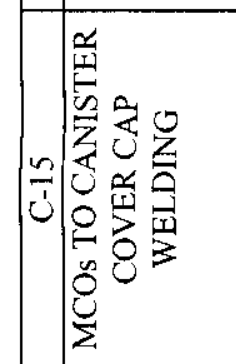 & 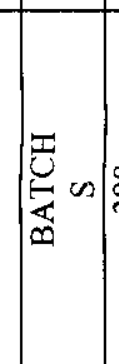 & & 18 & 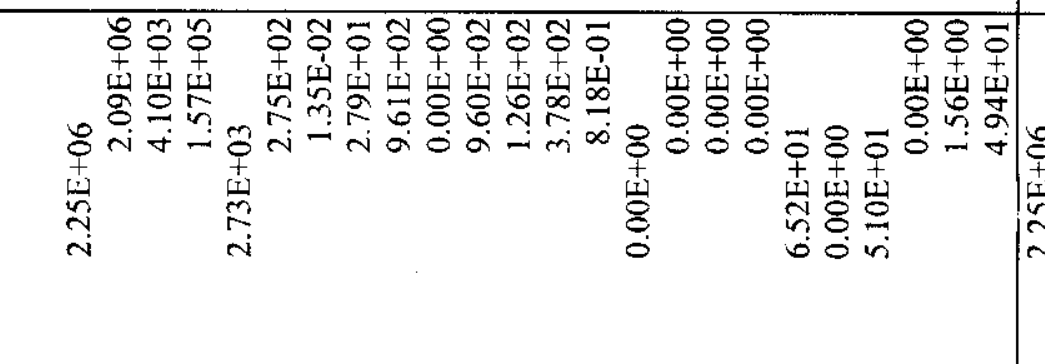 \\
\hline 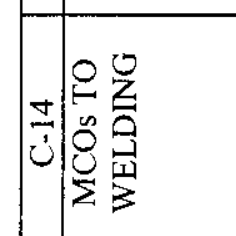 & 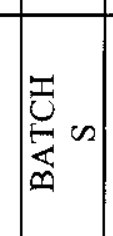 & $\begin{array}{c}\infty \\
\stackrel{0}{n}\end{array}$ & . & 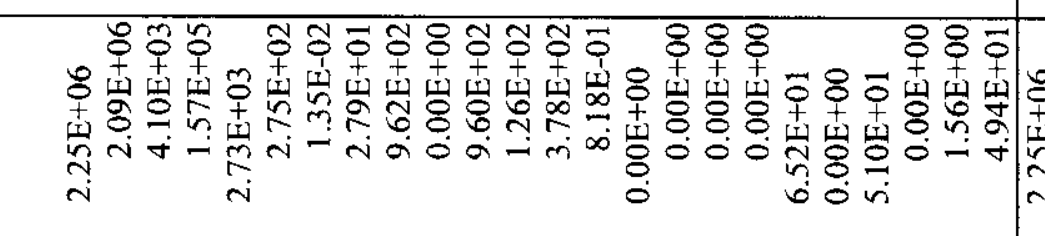 \\
\hline 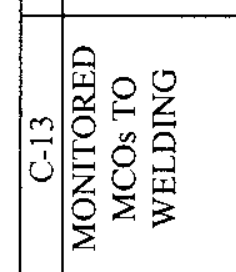 & 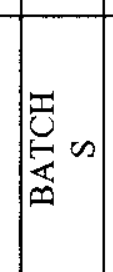 & $=\frac{8}{2}$ & '8: & 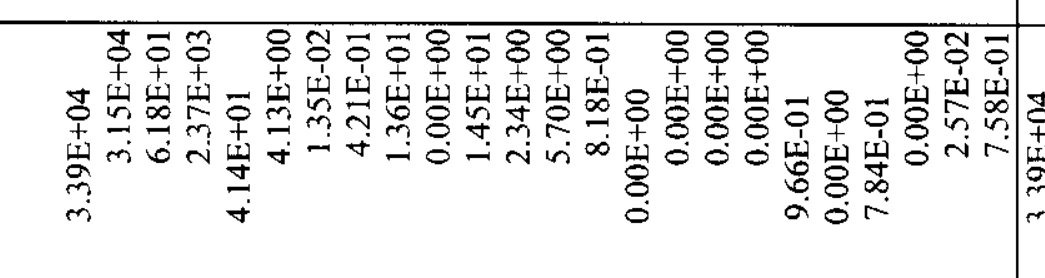 \\
\hline 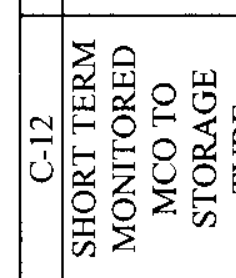 & 䗎。 & & 188 & 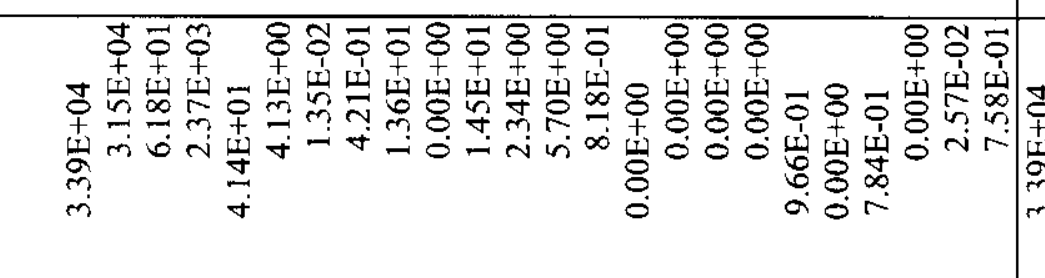 \\
\hline 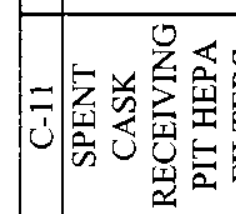 & 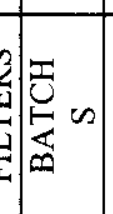 & & 畜 & 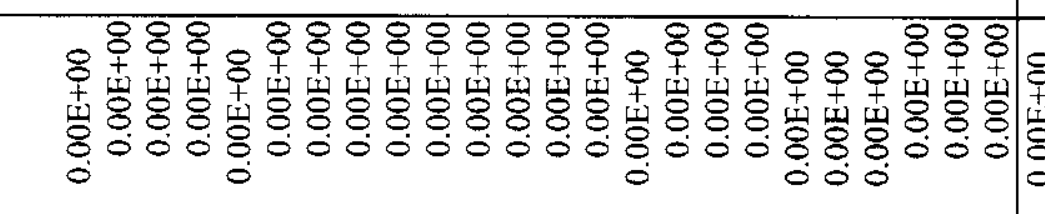 \\
\hline 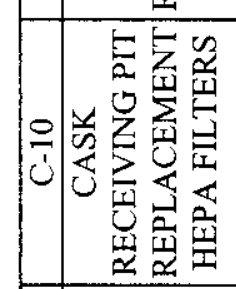 & 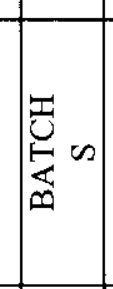 & & 怘 & 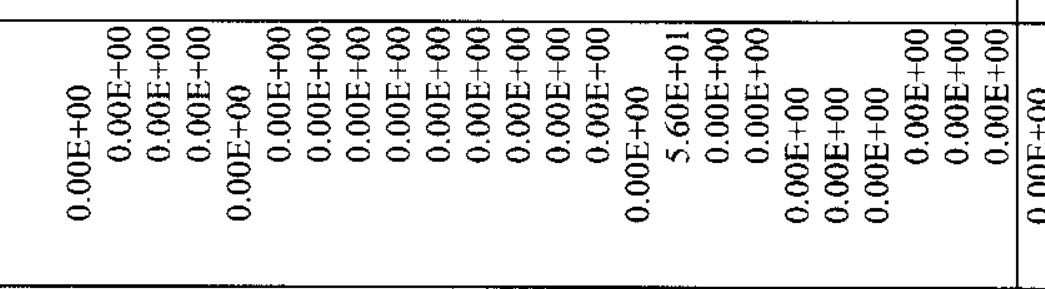 \\
\hline 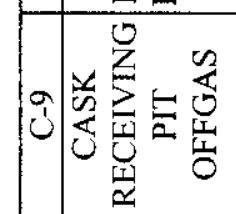 & 焉。 & & 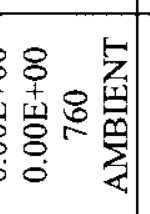 & 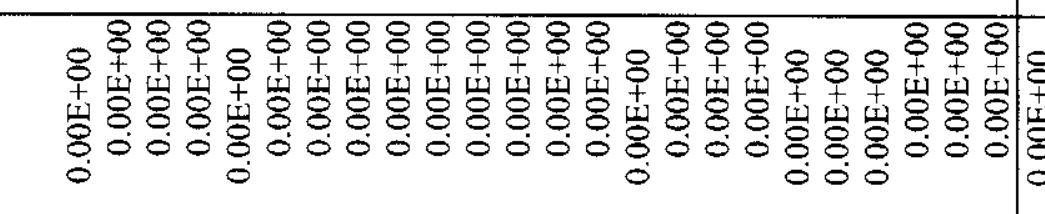 \\
\hline 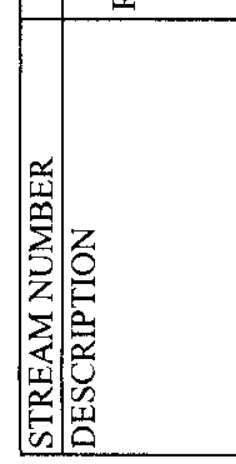 & 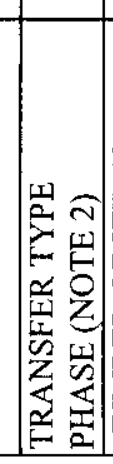 & 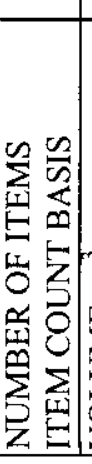 & 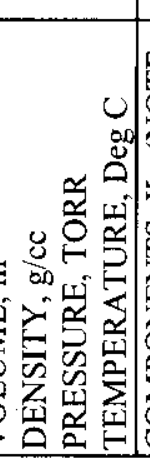 & 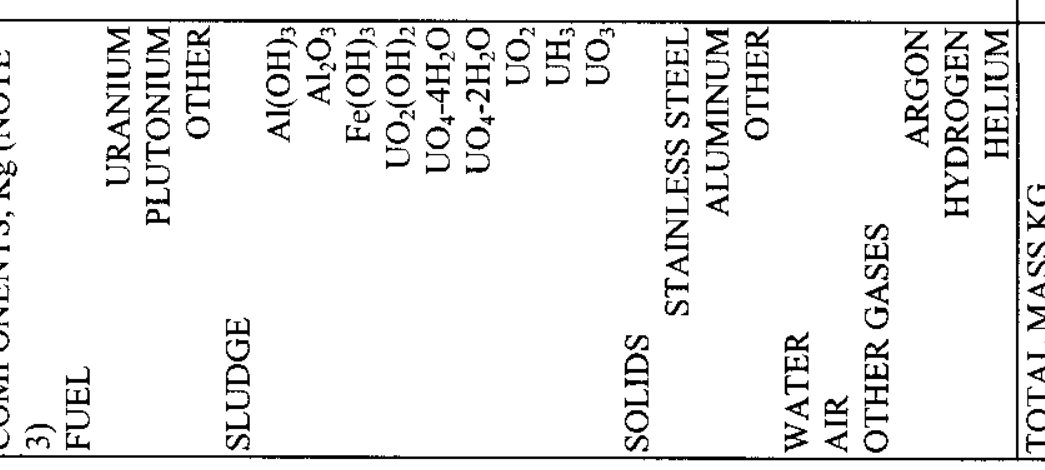 \\
\hline
\end{tabular}




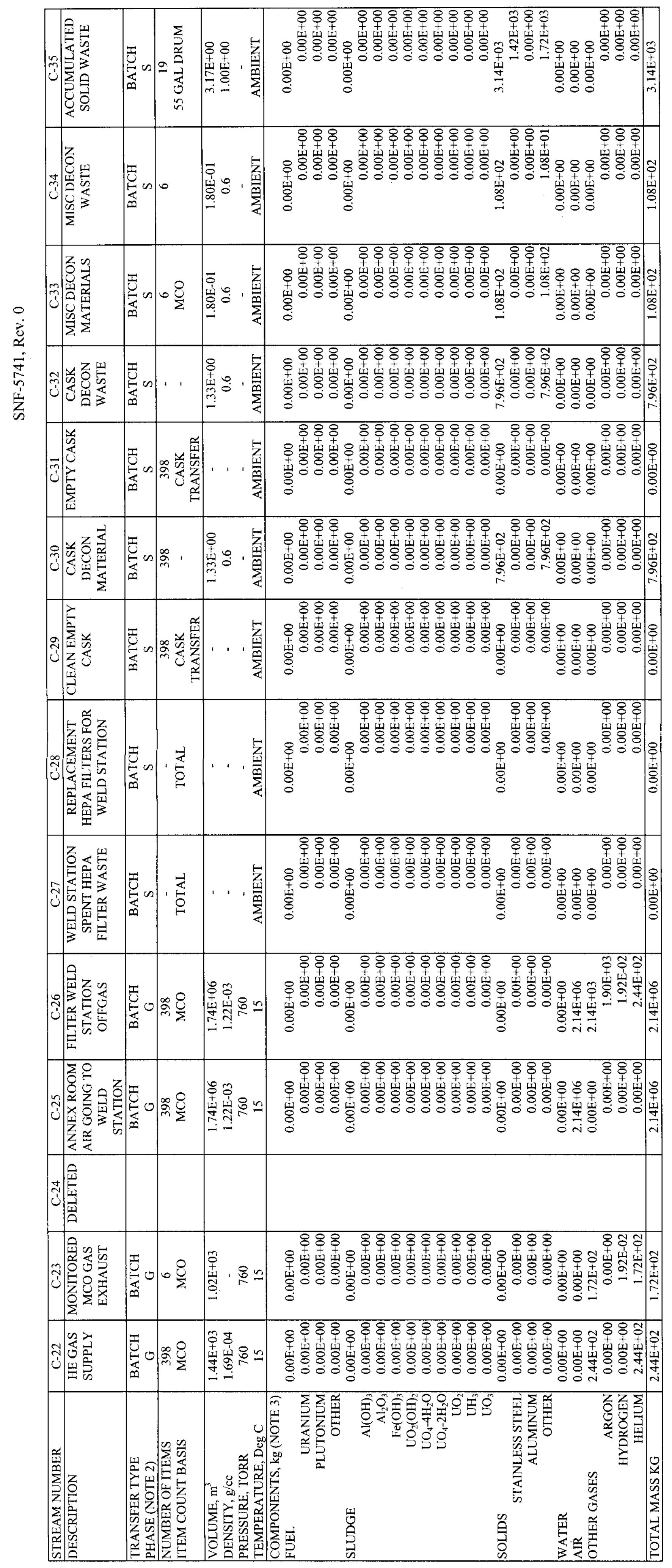




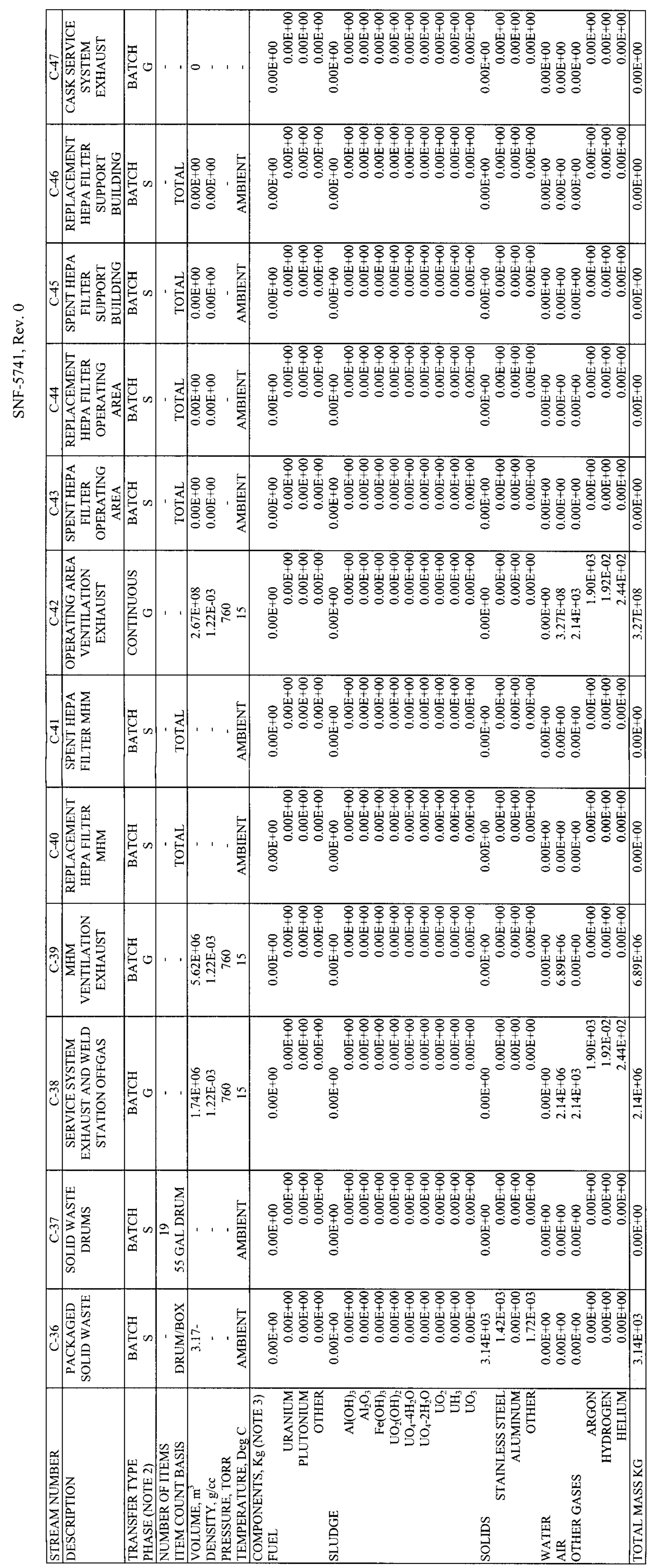


SNF-5741, Rev. 0

\begin{tabular}{|c|c|c|c|c|c|c|}
\hline STREAM NUMBER & C- -48 & C-49 & C- -50 & $\mathrm{C}-51$ & C-52 & $\mathrm{C}-53$ \\
\hline DESCRIPTION & $\begin{array}{l}\text { SOLID WASTE } \\
\text { BOXES }\end{array}$ & $\begin{array}{l}\text { ACCUMULATED } \\
\text { FILTER WASTE }\end{array}$ & $\begin{array}{l}\text { REPLACEMENT } \\
\text { SAMPLE HEPA } \\
\text { FILTER }\end{array}$ & $\begin{array}{c}\text { SPENT HEPA } \\
\text { FILTER FROM } \\
\text { SAMPLE HOOD }\end{array}$ & $\begin{array}{c}\text { HELIUM } \\
\text { REINERTED } \\
\text { MONITORED MCOS } \\
\text { TO WELDING }\end{array}$ & $\begin{array}{c}\text { PRESSURE } \\
\text { SENDING AND } \\
\text { READOUT UNITS }\end{array}$ \\
\hline $\begin{array}{l}\text { TRANSFER TYPE } \\
\text { PHASE (NOTE 2) }\end{array}$ & $\begin{array}{c}\text { BATCH } \\
\mathrm{S} \\
\end{array}$ & $\begin{array}{c}\text { BATCH } \\
\mathrm{S} \\
\end{array}$ & $\begin{array}{c}\mathrm{BATCH} \\
\mathrm{S} \\
\end{array}$ & $\begin{array}{c}\text { BATCH } \\
\mathrm{S} \\
\end{array}$ & $\begin{array}{c}\text { BATCH } \\
\text { S/G }\end{array}$ & $\begin{array}{c}\text { BATCH } \\
\mathrm{S} \\
\end{array}$ \\
\hline $\begin{array}{l}\text { NUMBER OF ITEMS } \\
\text { ITEM COUNT BASIS }\end{array}$ & $\overline{-}-\bar{B}$ & BURIAL BOX & TOTAL & TOTAL & $\begin{array}{c}6 \\
\mathrm{MCO} \\
\end{array}$ & $\begin{array}{c}398 \\
\mathrm{MCO} \\
\end{array}$ \\
\hline $\begin{array}{l}\text { VOLUME, } \mathbf{m}^{3} \\
\text { DENSITY, g/cc } \\
\text { PRESSURE, TORR } \\
\text { TEMPERATURE, Deg } \\
\text { C }\end{array}$ & $\begin{array}{c}- \\
- \\
- \\
\text { AMBIENT }\end{array}$ & $\begin{array}{c}- \\
- \\
- \\
\text { AMBIENT }\end{array}$ & $\begin{array}{c}- \\
- \\
- \\
\text { AMBIENT }\end{array}$ & $\begin{array}{c}- \\
- \\
- \\
\text { AMBIENT }\end{array}$ & $\begin{array}{c}- \\
1400 \\
60\end{array}$ & $\begin{array}{c}3.63 \mathrm{E}-02 \\
- \\
- \\
\text { AMBIENT }\end{array}$ \\
\hline $\begin{array}{l}\text { COMPONENTS, kg } \\
\text { (NOTE 3) }\end{array}$ & & & & & & \\
\hline FUEL & $0.00 \mathrm{E}+00$ & $\begin{array}{c}0.00 \mathrm{E}+00 \\
0.00 \mathrm{E}+00\end{array}$ & $\begin{array}{c}0.00 \mathrm{E}+00 \\
0.00 \mathrm{E}+00\end{array}$ & $\begin{array}{c}0.00 \mathrm{E}+00 \\
0.00 \mathrm{E}+00\end{array}$ & $3.39 \mathrm{E}+04$ & $0.00 \mathrm{E}+00$ \\
\hline PLUTONIUM & $0.00 \mathrm{E}+00$ & $0.00 \mathrm{E}+00$ & $0.00 \mathrm{E}+00$ & $0.00 \mathrm{E}+00$ & $\begin{array}{l}3.15 \mathrm{CT} 4 \\
6.18 \mathrm{E}+01\end{array}$ & $0.00 \mathrm{E}+00$ \\
\hline OTHER & $0.00 \mathrm{E}+00$ & $0.00 \mathrm{E}+00$ & $0.00 \mathrm{E}+00$ & $0.00 \mathrm{E}+00$ & $2.37 \mathrm{E}+03$ & $0.00 \mathrm{E}+00$ \\
\hline SLUDGE & $0.00 \mathrm{E}+00$ & $0.00 \mathrm{E}+00$ & $0.00 \mathrm{E}+00$ & $0.00 \mathrm{E}+00$ & $4.14 \mathrm{E}+01$ & $0.00 \mathrm{E}+00$ \\
\hline $\mathrm{Al}(\mathrm{OH})_{3}$ & $0.00 \mathrm{E}+00$ & $0.00 \mathrm{E}+00$ & $0.00 \mathrm{E}+00$ & $0.00 \mathrm{E}+00$ & 4. $13 E+00$ & $0.00 \mathrm{E}+00$ \\
\hline $\mathrm{Al}_{2} \mathrm{O}_{3}$ & $0.00 \mathrm{E}+00$ & $0.00 \mathrm{E}+00$ & $0.00 \mathrm{E}+00$ & $0.00 \mathrm{E}+00$ & $1.35 \mathrm{E}-02$ & $0.00 E+00$ \\
\hline $\mathrm{Fe}(\mathrm{OH})_{3}$ & $0.00 \mathrm{E}+00$ & $0.00 \mathrm{E}+00$ & $0.00 \mathrm{E}+00$ & $0.00 \mathrm{E}+00$ & $4.21 \mathrm{E}-01$ & $0.00 \mathrm{E}+00$ \\
\hline $\mathrm{UO}_{2}(\mathrm{OH})_{2}$ & $0.00 \mathrm{E}+00$ & $0.00 \mathrm{E}+00$ & $0.00 \mathrm{E}+00$ & $0.00 \mathrm{E}+00$ & $1.36 \mathrm{E}+01$ & $0.00 \mathrm{E}+00$ \\
\hline $\mathrm{UO}_{4}-4 \mathrm{H}_{2} \mathrm{O}$ & $0.00 \mathrm{E}+00$ & $0.00 \mathrm{E}+00$ & $0.00 \mathrm{E}+00$ & $0.00 \mathrm{E}+00$ & $0.00 \mathrm{E}+00$ & $0.00 \mathrm{E}+00$ \\
\hline $\mathrm{UO}_{4}-2 \mathrm{H}_{2} \mathrm{O}$ & $0.00 \mathrm{E}+00$ & $0.00 \mathrm{E}+00$ & $0.00 \mathrm{E}+00$ & $0.00 \mathrm{E}+00$ & $1.45 \mathrm{E}+01$ & $0.00 \mathrm{E}+00$ \\
\hline $\mathrm{UO}_{2}$ & $0.00 \mathrm{E}+00$ & $0.00 \mathrm{E}+00$ & $0.00 \mathrm{E}+00$ & $0.00 \mathrm{E}+00$ & $2.34 \mathrm{E}+00$ & $0.00 \mathrm{E}+00$ \\
\hline $\mathrm{UH}_{3}$ & $0.00 \mathrm{E}+00$ & $0.00 \mathrm{E}+00$ & $0.00 \mathrm{E}+00$ & $0.00 \mathrm{E}+00$ & $5.70 \mathrm{E}+00$ & $0.00 \mathrm{E}+00$ \\
\hline $\mathrm{UO}_{3}$ & $0.00 \mathrm{E}+00$ & $0.00 \mathrm{E}+00$ & $0.00 \mathrm{E}+00$ & $0.00 \mathrm{E}+00$ & $8.18 \mathrm{E}-01$ & $0.00 \mathrm{E}+00$ \\
\hline SOLIDS & $0.00 \mathrm{E}+00$ & $0.00 \mathrm{E}+00$ & $0.00 \mathrm{E}+00$ & $0.00 \mathrm{E}+00$ & $0.00 \mathrm{E}+00$ & $7.44 \mathrm{E}+01$ \\
\hline STAINLESS STEEL & $0.00 \mathrm{E}+00$ & $0.00 \mathrm{E}+00$ & $0.00 \mathrm{E}+00$ & $0.00 \mathrm{E}+00$ & $0.00 \mathrm{E}+00$ & $7.44 \mathrm{E}+01$ \\
\hline ALUMINUM & $0.00 \mathrm{E}+00$ & $0.00 \mathrm{E}+00$ & $0.00 \mathrm{E}+00$ & $0.00 \mathrm{E}+00$ & $0.00 \mathrm{E}+00$ & $0.00 \mathrm{E}+00$ \\
\hline OTHER & $0.00 \mathrm{E}+00$ & $0.00 \mathrm{E}+00$ & $0.00 \mathrm{E}+00$ & $0.00 \mathrm{E}+00$ & $0.00 \mathrm{E}+00$ & $0.00 \mathrm{E}+00$ \\
\hline WATER & $0.00 \mathrm{E}+00$ & $0.00 \mathrm{E}+00$ & $0.00 \mathrm{E}+00$ & $0.00 \mathrm{E}+00$ & $9.66 \mathrm{E}-01$ & $0.00 \mathrm{E}+00$ \\
\hline & $0.00 \mathrm{E}+00$ & $0.00 \mathrm{E}+00$ & $0.00 \mathrm{E}+00$ & $0.00 \mathrm{E}+00$ & $0.00 \mathrm{E}+00$ & $0.00 \mathrm{E}+00$ \\
\hline OTHER GASES & $0.00 \mathrm{E}+00$ & $0.00 \mathrm{E}+00$ & $0.00 \mathrm{E}+00$ & $0.00 \mathrm{E}+00$ & $7.94 \mathrm{E}-01$ & $0.00 \mathrm{E}+00$ \\
\hline ARGON & $0.00 \mathrm{E}+00$ & $0.00 \mathrm{E}+00$ & $0.00 \mathrm{E}+00$ & $0.00 \mathrm{E}+00$ & $0.00 \mathrm{E}+00$ & $0.00 \mathrm{E}+00$ \\
\hline HYDROGEN & $0.00 \mathrm{E}+00$ & $0.00 \mathrm{E}+00$ & $0.00 \mathrm{E}+00$ & $0.00 \mathrm{E}+00$ & $1.38 \mathrm{E}-02$ & $0.00 \mathrm{E}+00$ \\
\hline HELIUM & $0.00 E+00$ & $0.00 \mathrm{E}+00$ & $0.00 \mathrm{E}+00$ & $0.00 \mathrm{E}+00$ & $7.80 \mathrm{E}-01$ & $0.00 \mathrm{E}+00$ \\
\hline TOTAL MASS KG & $0.00 \mathrm{E}+00$ & $0.00 \mathrm{E}+00$ & $0.00 \mathrm{E}+00$ & $0.00 \mathrm{E}+00$ & $3.39 \mathrm{E}+04$ & $7.44 \mathrm{E}+01$ \\
\hline
\end{tabular}




\section{REVIEW CHECKLIST}

Document Reviewed:

SNF-5741, SNF Project Canister Storage Building PFD Mass Balance Calculations

Ruo

Scope of Review:

Technical Review of Mass Balance Calculations

\section{Yes No NA}

Q $\bigcirc$ * Previous reviews complete and cover analysis, up to scope of this review, with no gaps.

$\bigotimes \bigcirc \bigcirc$ Problem completely defined.

$\bigcirc \bigcirc \quad$ Accident scenarios developed in a clear and logical manner.

$\bigcirc \bigcirc \quad$ Necessary assumptions explicitly stated and supported.

$\bigcirc \bigcirc \quad$ Computer codes and data files documented.

Q $\bigcirc \quad$ Data used in calculations explicitly stated in document.

$\otimes \bigcirc \quad$ Data checked for consistency with original source information as applicable.

Q $\bigcirc$ Mathematical derivation checked including dimensional consistency of results.

$\bigcirc \bigcirc$ Models appropriate and used within range of validity or use outside range of established validity justified.

\$ $\bigcirc$ Hand calculations checked for errors. Spreadsheet results should be treated exactly the same as hand calculations.

$\bigcirc \bigcirc \quad$ Software input correct and consistent with document reviewed.

$\bigcirc \quad Q \quad$ Software output consistent with input and with results reported in document reviewed.

$\$ \bigcirc \bigcirc$ Limits/criteria/guidelines applied to analysis results are appropriate and referenced.

\& $\bigcirc \quad$ Limits/criteria/guidelines checked against references.

$\otimes \bigcirc \bigcirc$ Conclusions consistent with analytical results and applicable limits.

\& $\bigcirc$ Results and conclusions address all points required in the problem statement.

$\bigcirc \bigcirc \quad$ Format consistent with appropriate NRC Regulatory Guide or other standards.

$\bigcirc \bigcirc *$ Review calculations, comments, and/or notes are attached.

Q $\bigcirc \quad$ Document approved.

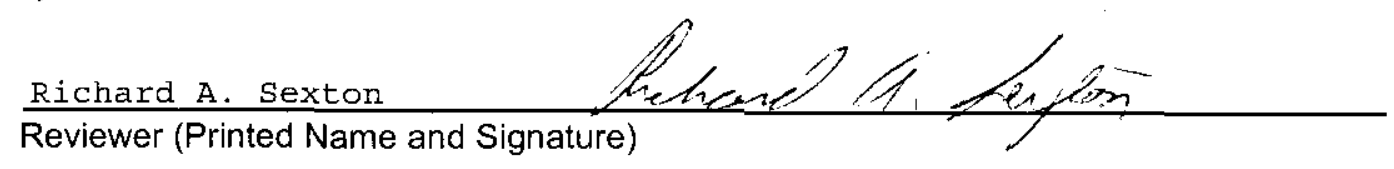

$\frac{1 / 25 / 00}{\text { Date }}$

*Any calculations, comments, or notes generated as part of this review should be signed, dated and attached to this checklist. Such material should be labeled and recorded in such a manner as to be intelligible to a technically qualified third party. 
Qocumen S SNE 5241

$3<$ Haltione

Recto. Vंerso

\begin{tabular}{l|l|}
\hline$D I S T$ & - \\
\hline$E D T$ & - \\
\hline Cover & - \\
\hline Cover & disclarmin
\end{tabular}$:$

\begin{tabular}{c|c} 
i: & ii \\
\hline iii & $=$ \\
\hline 1 & 2 \\
\hline 1 & \\
\hline 39 & 140 \\
\hline 41 & 1
\end{tabular}

$A-11$

$A-2$

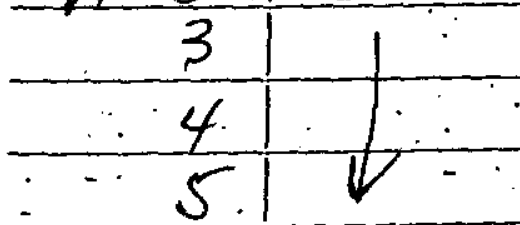

$A-6$.

Cklest
Foldout

Verso

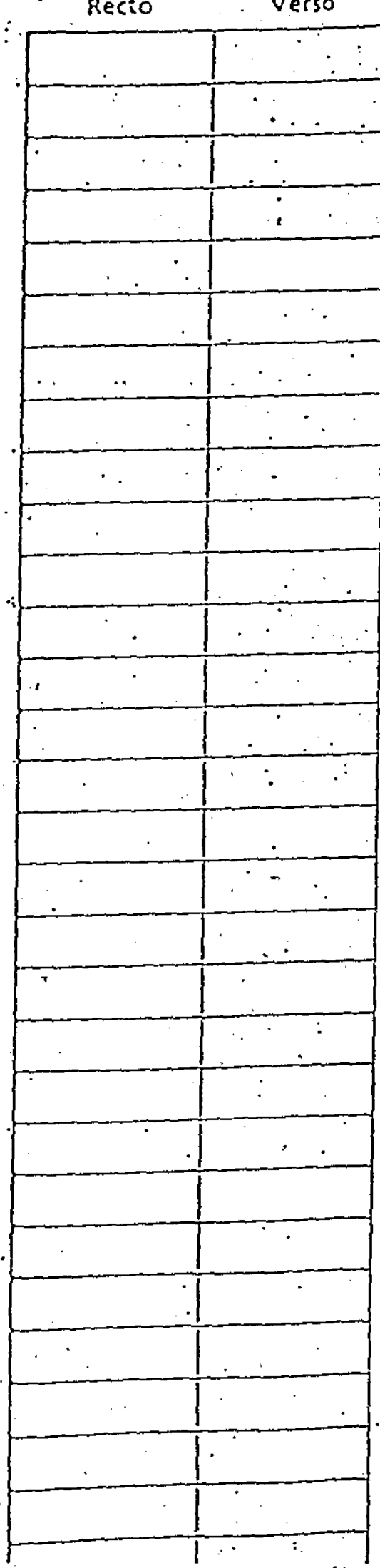

Page $\therefore \quad l$ of. $L$ Number of Originals

Reduction

Recro $\because$ Verso

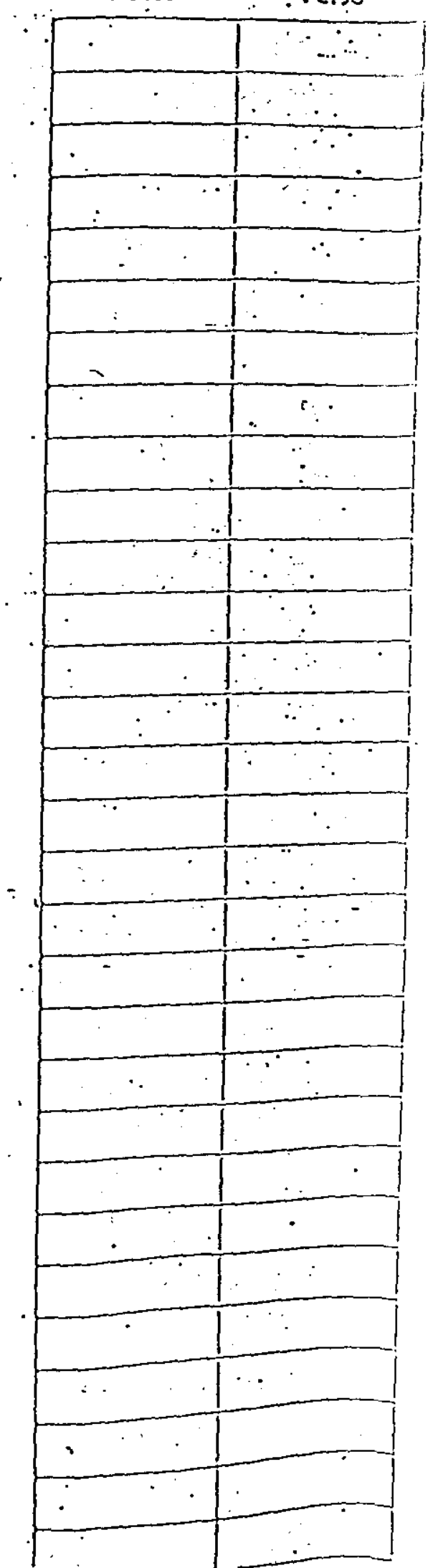




\section{DIST'RIBUTION SHEET}

\begin{tabular}{|c|c|c|c|c|c|}
\hline \multirow{2}{*}{$\begin{array}{l}\text { To } \\
\text { Distribution }\end{array}$} & \multirow{2}{*}{\multicolumn{3}{|c|}{$\begin{array}{l}\text { From } \\
\text { Process Engineering }\end{array}$}} & \multicolumn{2}{|l|}{ Page 1 of 1} \\
\hline & & & & \multicolumn{2}{|l|}{ Date $2 / 2 / 00$} \\
\hline \multicolumn{4}{|l|}{ Project Title/Work Order } & \multicolumn{2}{|c|}{ EDT No. 628526} \\
\hline \multicolumn{4}{|c|}{$\begin{array}{l}\text { Spent Nuclear Fuel Project Canister Storage Building Process Flow Diagram Mass Balance } \\
\text { Calculations }\end{array}$} & \multicolumn{2}{|l|}{ ECN No. N/A } \\
\hline Name & MSIN & $\begin{array}{c}\text { Text } \\
\text { With All } \\
\text { Attach. }\end{array}$ & $\begin{array}{l}\text { Text } \\
\text { Only }\end{array}$ & $\begin{array}{l}\text { Attach./ } \\
\text { Appendix } \\
\text { Only }\end{array}$ & $\begin{array}{l}\text { EDT/ECN } \\
\text { Only }\end{array}$ \\
\hline
\end{tabular}

Spent Nuclear Fuel Project

G. D. Bazinet

D. M. Black

S. B. Carter

D. L. Cooley

J. R. Frederickson

R. L. Garrett

M. J. Klem

S. A. Krieg

F. W. Moore

S. S. Moss

A. L. Pajunen

O. M. Serrano

R. A. Sexton

V. D. Zarasua

SNF Project Files

SNF Project Procedures

SNF Project Training

$\begin{array}{ll}\text { S8-06 } & X \\ \text { R3-86 } & X \\ \text { S8-05 } & X \\ \text { R3-86 } & X \\ \text { R3-86 } & \text { X } \\ \text { R3-26 } & \text { X } \\ \text { R3-86 } & \text { X } \\ \text { S8-06 } & \text { X } \\ \text { R3-86 } & \text { X } \\ \text { R3-11 } & \text { X } \\ \text { R3-86 } & \text { X } \\ \text { S2-44 } & \text { X } \\ \text { R3-86 } & \text { X } \\ \text { S2-44 } & \text { X } \\ \text { R3-11 } & \text { X } \\ \text { X3-86 } & \\ \text { S2-45 } & \end{array}$

$\mathrm{X}$ 